\title{
CLOSURE REPORT FOR CORRECTIVE ACTION UNIT 523: HOUSEKEEPING WASTE, NEVADA TEST SITE, NEVADA
}

\author{
Prepared for: \\ U.S. Department of Energy \\ National Nuclear Security Administration \\ Nevada Site Office \\ Work Performed Under Contract No. DE-AC08-96NV11718
}

Controlled Copy No:

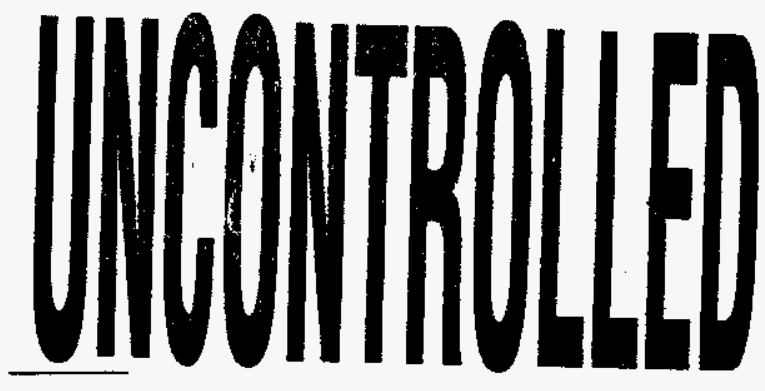

Revision: 0

November 2003 


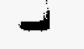




\section{CLOSURE REPORT FOR CORRECTIVE ACTION UNIT 523: HOUSEKEEPING WASTE, NEVADA TEST SITE, NEVADA}

Approved by:

Janet Appenzeller-Wing, Project manager

Industrial Sites Project

Approved by: Gom Rew

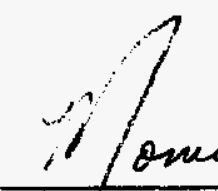

Runofe c. Wycoff, Director

Environmental Restoration Division
Date: $1 /-12-03$

Date: $11 / 12 / 03$ 


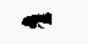




\section{TABLE OF CONTENTS}

ACRONYMS AND ABBREVIATIONS …...................................................................... vii

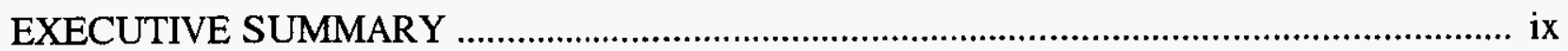

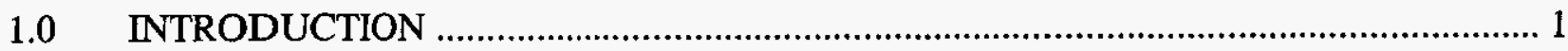

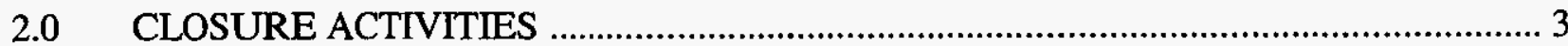

$2.1 \quad$ DESCRIPTION OF CLOSURE ACTIVITIES ……........................................ 3

2.1.1 Preplanning and Site Preparation .............................................................. 3

2.2 SITE CHARACTERIZATION ACTIVITIES …….............................................. 3

2.2.1 CAS 12-22-20: Drum ……………………................................. 5

2.2.2 CAS 18-22-24: Bottles - Lithium Bromide ............................................. 5

2.2.3 CAS 19-22-31: Can ...................................................................... 5

2.2.4 CAS 20-22-27: Bucket ……………………….................................. 5

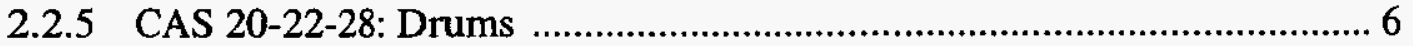

2.2.6 CAS 20-22-29: Bucket ..................................................................... 6

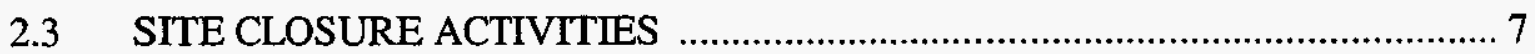

2.3.1 CAS 04-22-04: Drums ................................................................. 7

2.3.2 CAS 12-22-20: Drum ....................................................................... 7

2.3.3 CAS 12-30-16: Manhole for Gas/Air Line ……....................................... 7

2.3.4 CAS 16-22-29: Drum ................................................................... 7

2.3.5 CAS 18-22-24: Bottles - Lithium Bromide ………………………............ 7

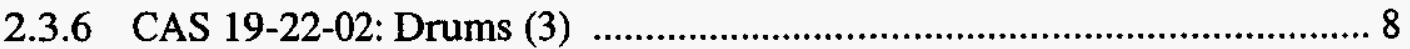

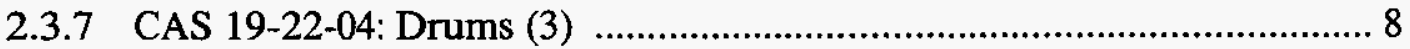

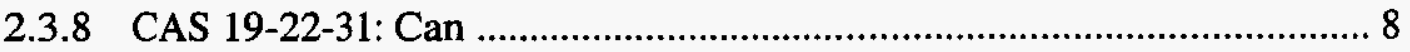

2.3.9 CAS 19-22-32: Can; Debris .............................................................. 8

2.3.10 CAS 20-22-03: Drums ................................................................... 8

2.3.11 CAS 20-22-10: Drum ..................................................................... 9

2.3.12 CAS 20-22-20: Drum ………….................................................... 9

2.3.13 CAS 20-22-27: Bucket ................................................................... 9

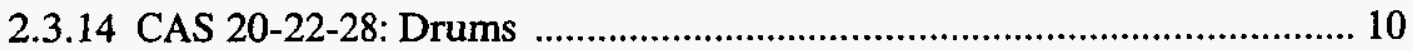

2.3.15 CAS 20-22-29: Bucket ................................................................... 10

2.3.16 CAS 20-26-01: Lead Pellets .......................................................... 10

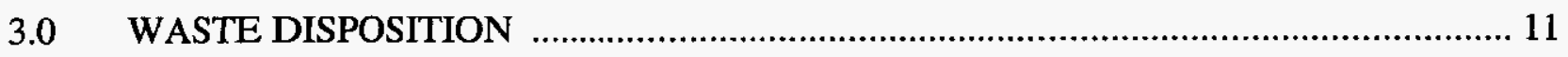

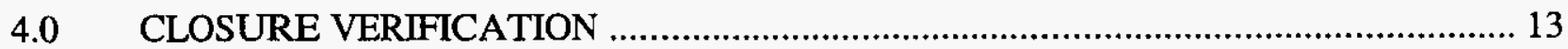

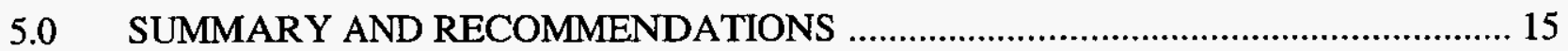

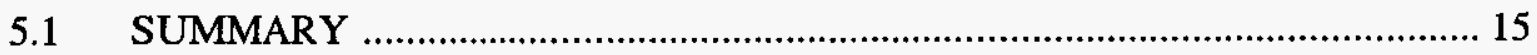

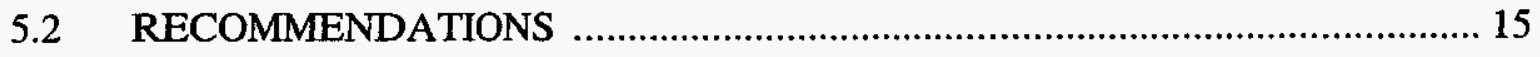

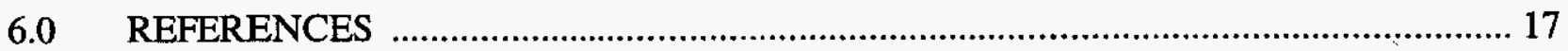




\section{TABLE OF CONTENTS (continued)}

\section{FIGURES}

FIGURE 1 - CAU 523 SITE LOCATION MAP

2

\section{TABLES}

TABLE 1 - SUMMARY OF ANALYTICAL RESULTS FOR CHARACTERIZATION

SAMPLES

TABLE 2 - SUMMARY OF ANALYTICAL RESULTS FOR VERIFICATION SOIL SAMPLES 9

\section{APPENDICES}

APPENDIX A: SAMPLE ANALYTICAL RESULTS

APPENDIX B: SECTORED HOUSEKEEPING SITE CLOSURE VERIFICATION FORMS

APPENDIX C: NATIONAL ENVIRONMENTAL POLICY ACT EVALUATION CHECKLIST

APPENDIX D: DOCUMENTATION FOR CAS 12-30-16: MANHOLE FOR GAS/AIR LINE DISTRIBUTION LIST 


\section{ACRONYMS AND ABBREVIATIONS}

\begin{tabular}{|c|c|}
\hline $\mathrm{BN}$ & Bechtel Nevada \\
\hline CAS & Corrective Action Site(s) \\
\hline CAU & Corrective Action Unit \\
\hline $\mathrm{COC}$ & Contaminant(s) of Concern \\
\hline CFR & Code of Federal Regulations \\
\hline DOE/NV & U.S. Department of Energy Nevada Operations Office \\
\hline EPA & U.S. Environmental Protection Agency \\
\hline ER & Environmental Restoration \\
\hline FFACO & Federal Facility Agreement and Consent Order \\
\hline FRC & Free Release Criteria \\
\hline $\mathrm{ft}^{3}$ & Cubic foot(feet) \\
\hline gal & Gallon(s) \\
\hline $\mathbf{L}$ & Liter(s) \\
\hline $\mathrm{m}^{3}$ & Cubic meter(s) \\
\hline $\mathrm{mg} / \mathrm{kg}$ & Milligram(s) per kilogram \\
\hline $\mathrm{mg} / \mathrm{L}$ & Milligram(s) per liter \\
\hline MSDS & Material Safety Data Sheet \\
\hline NAC & Nevada Administrative Code \\
\hline ND & Not Detected \\
\hline NDEP & Nevada Division of Environmental Protection \\
\hline NNSA/NSO & $\begin{array}{l}\text { U.S. Department of Energy, National Nuclear Security Administration Nevada } \\
\text { Site Office }\end{array}$ \\
\hline NS & Not Sampled \\
\hline NTS & Nevada Test Site \\
\hline PCBs & polychlorinated biphenyls \\
\hline $\mathrm{pCi} / \mathrm{g}$ & picoCuries per gram \\
\hline & parts per million \\
\hline
\end{tabular}




\section{ACRONYMS AND ABBREVIATIONS (continued)}

PRGs Preliminary Remediation Guidelines

RCRA Resource Conservation and Recovery Act

SAA Satellite Accumulation Area

SDG Sample Delivery Group

SVOC Semivolatile Organic Compound(s)

SWO Solid Waste Operations

TCLP Toxicity Characteristic Leaching Procedure

TPH Total Petroleum Hydrocarbons

VOC Volatile Organic Compound(s) 


\section{EXECUTIVE SUMMARY}

The 16 Corrective Action Sites (CASs) included in Corrective Action Unit 523 were closed by the following actions:

- CAS 04-22-04 (Drums): Clean-closed with no action necessary

- CAS 12-22-20 (Drum): Clean-closed

- CAS 12-30-16 (Manhole for Gas/Air Line): no action taken

- CAS 16-22-29 (Drum): Clean-closed with no action necessary

- $\quad$ CAS 18-22-24 (Bottles - Lithium Bromide): Clean-closed

- CAS 19-22-02 (Drums [3]): Clean-closed with no action necessary

- $\quad$ CAS 19-22-04 (Drums [3]): Clean-closed

- CAS 19-22-31 (Can): Clean-closed

- CAS 19-22-32 (Can; Debris): Clean-closed

- CAS 20-22-03 (Drums): Clean-closed with no action necessary

- CAS 20-22-10 (Drum): Clean-closed with no action necessary

- CAS 20-22-20 (Drum): Clean-closed with no action necessary

- $\quad$ CAS 20-22-27 (Bucket): Clean-closed

- $\quad$ CAS 20-22-28 (Drums): Clean-closed

- CAS 20-22-29 (Bucket): Clean-closed

- CAS 20-26-01 (Lead Pellets): Clean-closed

Note: CAS 12-30-16 (Manhole for Gas/Air Line) is part of the currently inactive 12-2 water transmission line running between the Area 12 Camp and the Area 2 Camp. The manhole contains an air/vacuum release valve. The manhole and materials inside are not waste material. For this reason, no action was taken to close this site; a Federal Facility Agreement and Consent Order modification to remove CAS 12-30-16 from CAU 355 and place it in CAU 5000 has been prepared, submitted, and is pending approval. 
Closure Report - CAU 523

Section: Executive Summary

Revision: 0

Date: November 2003

THIS PAGE INTENTIONALLY LEFT BLANK 


\subsection{INTRODUCTION}

This Closure Report documents the closure activities conducted for Corrective Action Unit (CAU) 523: Housekeeping Waste. CAU 523 is listed in Appendix III of the Federal Facility Agreement and Consent Order (FFACO, 1996) and consists of the following 16 Corrective Action Sites (CASs) located in Areas 4, 12, 16, 18, 19, and 20 of the Nevada Test Site (NTS) (Figure 1):

- $\quad$ CAS 04-22-04: Drums
- $\quad$ CAS 12-22-20: Drum
- $\quad$ CAS 12-30-16: Manhole for Gas/Air Line
- $\quad$ CAS 18-22-29: Drum
- $\quad$ CAS 19-22-24: Bottles - Lithium Bromide
- $\quad$ CAS 19-22-04: Drums (3)
- $\quad$ CAS 19-22-31: Can
- $\quad$ CAS 19-22-32: Can; Debris
- $\quad$ CAS 20-22-03: Drums
- $\quad$ CAS 20-22-10: Drum 20-22-20: Drum
- $\quad$ CAS 20-22-27: Bucket
- $\quad$ CAS 20-22-28: Drums
- $\quad$ CAS 20-22-29: Bucket
CAS 20-26-01: Lead Pellets

Note: CAS 12-30-16 (Manhole for Gas/Air Line) is part of the currently inactive 12-2 water transmission line running between the Area 12 Camp and the Area 2 Camp. The manhole contains an air/vacuum release valve. The manhole and materials inside are not waste material. For this reason, no action was taken to close this site; a FFACO modification to remove CAS 12-30-16 from CAU 355 and place it in CAU 5000 has been prepared, submitted, and is pending approval.

Closure activities consisted of closing each CAS by removing debris and/or material, disposing of the generated waste, and verifying that each site was clean-closed by visual inspection and/or laboratory analysis of soil verification samples.

Copies of analytical results are included in Appendix A. Copies of the Sectored Housekeeping Site Closure Verification Forms for each of the 16 CASs are included in Appendix B. 


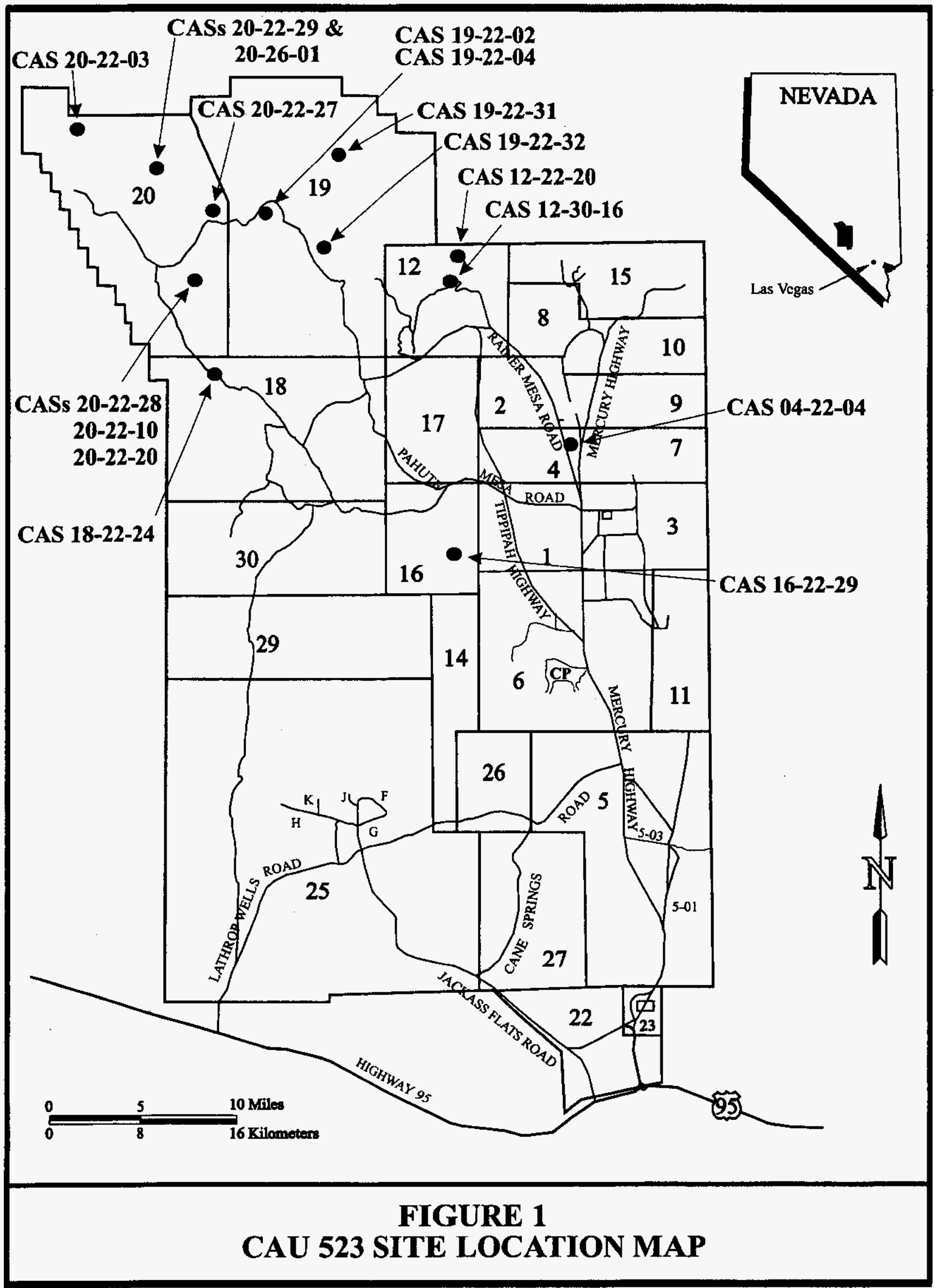




\subsection{CLOSURE ACTIVITIES}

This section details the specific corrective action activities completed during the closure of CAU 523: Housekeeping Waste.

\subsection{DESCRIPTION OF CLOSURE ACTIVITIES}

\subsubsection{Preplanning and Site Preparation}

Planning documents prepared prior to the beginning of closure activities included the following:

- $\quad$ Sectored Clean-up Work Plan For Housekeeping Category Waste Sites (U.S. Department of Energy, National Nuclear Security Administrations Nevada Site Office [NNSA/NSO], 2003).

- $\quad$ Generic Field Management Plan for Housekeeping Category Waste Sites (Bechtel Nevada [BN], 2002 ).

- Site-Specific Health and Safety Plan for Corrective Action Units 176, 346, 350, 351, 352 , $\underline{\&} 523$ Housekeeping Sites, Nevada Test Site, Nevada (BN, 2003).

- $\quad$ BN Solid Waste Operations (SWO) Work Packages.

- $\quad$ NNSA/NSO Real Estate/Operation Permit.

BN Environmental Restoration (ER) and SWO personnel held a pre-job field briefing on July 24, 2003 prior to the start of site-closure field activities.

\subsection{SITE CHARACTERIZATION ACTIVITIES}

None of the CAU 523 CASs were previously sampled for waste classification and process knowledge was insufficient to classify the potential waste at each site. During site vists, six sites were found to have potential waste streams requiring waste classification. Therefore, at CAS 12-22-20, 18-22-24, 19-22-31, 20-22-27, 20-22-28, and 20-22-29, samples were collected and analyzed to classify waste at the sites and determine the site closure activities. The samples were collected with clean disposable plastic scoops and placed in labeled sample containers secured with custody seals. The sample containers were placed on ice in a cooler and maintained at less than 4 degrees Celsius. They were transported under chain of custody to the BN Environmental Technical Services group in Mercury, Nevada, and shipped to an off-site laboratory for analysis. Table 1 summarizes the results of laboratory analyses performed for the characterization samples. Appendix A provides the analytical reports for the characterization samples. 


\section{TABLE 1 - SUMMARY OF ANALYTICAL RESULTS FOR}

\section{CHARACTERIZATION SAMPLES}

\begin{tabular}{|c|c|c|c|c|c|c|c|}
\hline 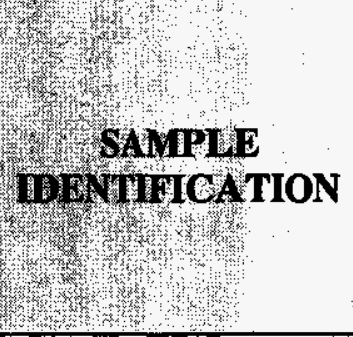 & 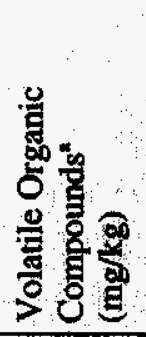 & 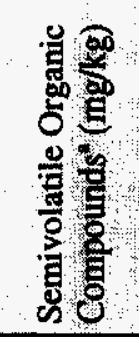 & 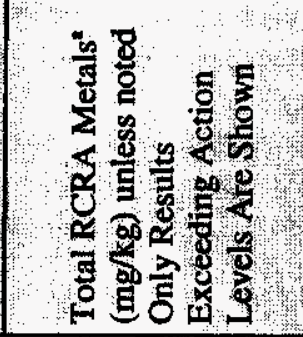 & 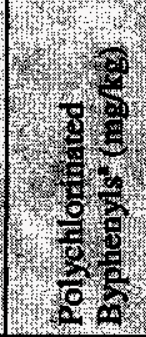 & 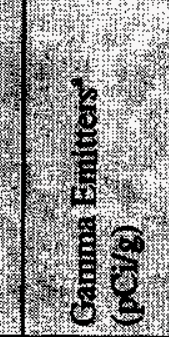 & 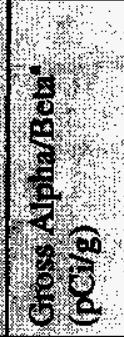 & 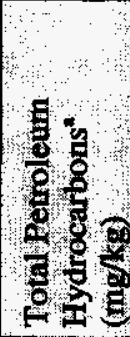 \\
\hline \multicolumn{8}{|c|}{ CAS 12-22-20 - Drum (Sample Delivery Group [SDG] V2004) } \\
\hline 122220-1 (Soil) & $<$ PRGs $^{\mathrm{b}}$ & $<$ PRGs & $<$ PRGs & ND & NS & NS & 38.0 \\
\hline \multicolumn{8}{|c|}{ CAS 18-22-24 - Bottles - Lithium Bromide (SDG V1978 and V1979) } \\
\hline $182224-1$ (Contents)* & $<$ RCRA $^{\mathrm{c}}$ & $<$ RCRA & Lead 246,000 & ND & $<F^{\prime d}$ & $<F R C$ & 222,000 \\
\hline $182224-2$ (Soil) & $<$ PRGs & $<$ PRGs & $<$ PRGs & ND & $<\mathrm{FRC}$ & NS & 90 \\
\hline \multicolumn{8}{|c|}{ CAS 19-22-31 - Can (SDG V1978 and V1979) } \\
\hline $192231+1$ (Soil) & $<$ PRGs & $<$ PRGs & $<$ PRGs & 0.27 & $<$ FRC & $<\mathrm{FRC}$ & 12,000 \\
\hline $192231-3$ (Soil) & $<$ PRGs & $<$ PRGs & $<$ PRGs & 0.28 & $<$ FRC & $<$ FRC & 1,890 \\
\hline \multicolumn{8}{|c|}{ CAS 20-22-27 - Bucket (SDG V1978, V1979 and V2029) } \\
\hline $202227-1$ (Soil) & $<$ PRGs & $<$ PRGs & $<$ PRGs & ND & $<$ FRC & $<$ FRC & ND \\
\hline $202227-2$ (Soil) & $<$ PRGs & $<$ PRGs & $<$ PRGs & ND & $\leq \mathrm{FRC}$ & $<$ FRC & 230 \\
\hline $202227-3$ (Contents)* & $<$ RCRA & $<$ RCRA & Lead 39,600 & ND & $<$ FRC & $<$ FRC & 147,000 \\
\hline $202227-4$ (Soil) & NS & NS & $\begin{array}{c}\text { TCLP Lead } 0.0385 \\
\mathrm{mg} / \mathrm{L}\end{array}$ & NS & NS & NS & NS \\
\hline \multicolumn{8}{|c|}{ CAS 20-22-28 - Drums (SDG V1978 and V1979) } \\
\hline 202228-1 (Contents) & $<$ RCRA & $<$ RCRA & $<$ RCRA & ND & $<$ FRC & NS & ND \\
\hline 202228-2 (Soil) & $<$ PRGs & $<$ PRGs & $<$ PRGs & ND & $<$ FRC & NS & ND \\
\hline 202228-3 (Soil) & $<$ PRGs & $<$ PRGs & $<$ PRGs & ND & $<$ FRC & NS & ND \\
\hline \multicolumn{8}{|c|}{ CAS 20-22-29 - Bucket (SDG V1978 and V1979) } \\
\hline 202229-1 (Soil) & $<$ PRGs & $<$ PRGs & $<$ PRGs & ND & $<$ FRC & NS & 30 \\
\hline
\end{tabular}

* Contents completely removed during sampling due to sample volume requirements.

- Volatile Organic Compounds (VOCs), Semivolatile Organic Compounds (SVOCs), total Resource Conservation and Recovery Act (RCRA) Metals, Polychlorinated Byphenyls (PCBs), Total Petroleum Hydrocarbons (TPH), Gamma, and Gross Alpha/Beta samples analyzed by U.S. Environmental Protection Agency (EPA) SW-846 method 8260, 8270, 6010 and 7470, 8082, 8015 modified, 901.1 and 906.0 respectively (EPA, 1996). Toxicity Characteristic Leaching Procedure (TCLP) RCRA metals were analyzed by 1311/6010 and 1311/7470. See analytical reports in Appendix A for laboratory detection limits.

b $<P R G s=$ Analytical results indicated that the levels of contaminants were less than the EPA Region IX Preliminary Remediation Goals (PRGs) for industrial soils (EPA, 2002).

- $\angle R C R A=$ Analytical results indicated that the waste was less than levels for hazardous waste according to Title 40 Code of Federal Regulations (CFR) Part 261.24 (CFR, 2002a).

- $\angle$ FRC = Analytical results were less than Nevada/Yucca Mountain Project Radiplogical Control Manual free release criteria for radioactive material/waste (U.S. Department of Energy/Nevada Operation Office [DOE/NV], 2000b).

Definitions:

$\mathrm{mg} / \mathrm{L}=$ Milligram(s) per liter

$\mathrm{mg} / \mathrm{kg}=$ Milligrams(s) per kilogram

$\mathrm{ND}=$ Not Detected at laboratory reporting limit or less than laboratory Minimum Detectable Activity. See Appendix A for limits.

NS $=$ Not Sampled

$\mathrm{pCi} / \mathrm{g}=$ picocuries per gram

ppm $=$ parts per million 


\subsubsection{CAS 12-22-20: Drum}

One empty 208-liter (L) (55-gallon [gal]) antifreeze drum was located at the site. One sample (122220-1) was collected from the soil directly beneath the drum. The sample was analyzed for volatile organic compounds (VOCs), semivolatile organic compounds (SVOCs), Resource Conservation and Recovery Act (RCRA) metals, total petroleum (gasoline, diesel, and motor oil range) hydrocarbons (TPH), polychlorinated biphenyls (PCBs), and gamma emitters. The analytical results from the soil below the drum were below U.S. Environmental Protection Agency (EPA) Preliminary Remediation Goals (PRGs) for industrial soils (EPA, 2002) and State of Nevada TPH action levels (Nevada Administrative Code [NAC], 2003). The empty drum was determined to be ordinary waste. The analytical data summary for the sample is included in Appendix A.

\subsubsection{CAS 18-22-24: Bottles - Lithium Bromide}

The Lithium Bromide bottles had been previously removed from the site. However, one 11.3-L (3-gal) bucket of Drill Collar Compound was found. One quarter of the bucket contained compound residue with the remainder filled with soil. One sample (182224-1) was collected from the compound inside the bucket and one sample (182224-2) was collected from the soil directly beneath the bucket. The samples were analyzed for VOCs, SVOCs, RCRA metals, TPH, PCBs, gamma emitters, and gross alpha/beta radioactivity (182224-1 only). The analytical results indicated that the bucket contents contained elevated concentrations of total lead and TPH. However, the contents of the bucket were completely depleted by sampling and the empty bucket was determined to be ordinary waste.

Analytical results presented in Table 1 indicate that the soil beneath the bucket did not exceed the State of Nevada action level for TPH (NAC, 2003), the PRGs (EPA, 2002) or action levels for any other Contaminants of Concern (COCs). Consequently, no soil was required to be removed from the site. Analytical data summaries for the samples are included in Appendix A.

\subsubsection{CAS 19-22-31: Can}

Three empty cans were located at the site in a small pile. One soil sample (192231-1) was collected from below the cans and analyzed for VOCs, SVOCs, RCRA metals, TPH, PCBs, gamma emitters, and gross alpha/beta radioactivity. The analytical results (Table 1) indicated that soil beneath the cans exceeded the State of Nevada action level for TPH (NAC, 2003) and that PCBs were present in the soil at concentrations of less than one milligram per kilogram $(\mathrm{mg} / \mathrm{kg})$. Therefore, the soil below the cans required additional hand excavation and verification sampling during the site closure activities (Section 2.3.8). The empty cans were determined to be ordinary waste. The analytical data summary for the sample is included in Appendix A.

\subsubsection{CAS 20-22-27: Bucket}

One 19-L (5-gal) bucket and one empty 208-L (55-gal) drum were located at the site. The bucket contained a small amount of a grease-like substance. One sample (202227-3) was collected from the contents of the bucket. The contents of the bucket were completely removed due to the sample volume requirements. One sample (202227-1) was collected from soil beneath 
the bucket, and one sample (202227-2) was collected from the soil beneath the empty drum. The samples were analyzed for VOCs, SVOCs, RCRA metals, TPH, PCBs, gamma emitters, and gross alpha/beta radioactivity.

The analytical results indicated elevated total lead and TPH concentrations in the contents of the bucket and elevated TPH concentrations in the soil beneath the empty drum. All remaining analytical results were either below the appropriate action level or laboratory method detection levels. Therefore, the soil below the drum required additional hand excavation and verification sampling during the site closure activities due to the elevated TPH concentration (Section 2.3.13). To further characterize the soil to be excavated, one additional sample (202227-4) was collected from the area of elevated TPH and analyzed for Toxicity Characteristic Leaching Procedure (TCLP) lead. Based on the analytical results, the soil to be excavated was not hazardous for lead, and the soil was determined to be hydrocarbon waste. The empty containers were determined to be ordinary waste. Analytical data summaries for the samples are included in Appendix A.

\subsubsection{CAS 20-22-28: Drums}

Three 113.5-L (30-gal) drums were located at the site. One of the drums was one quarter full of yellow plaster-like chips. The remaining two drums were empty. One sample (202228-1) was collected from the contents of the drum, while one soil sample was collected from beneath each of the two empty drums (samples 202228-2 and 202228-3). The samples were analyzed for VOCs, SVOCs, RCRA metals, TPH, PCBs, and gamma emitters. The analytical results indicated that the drum contents were not hazardous waste as defined by Title 40 Code of Federal Regulations (CFR) Part 261.24 (CFR, 2002a), TPH waste as defined by Part 445A.2272 of the NAC (NAC, 2003), radioactive waste (U.S. Department of Energy Nevada Operations Office [DOE/NV], 2000b), or Toxic Substance Control Act PCB waste per 40 CFR Part 761 (CFR, 2002b). Based on the analytical results, the drums and their contents were determined to be ordinary waste. Additionally, the analytical results indicated that concentrations in the soil were either below the appropriate action levels or laboratory method detection levels. Therefore, no soil was required to be removed from the site. Analytical data summaries for the samples are included in Appendix A.

\subsubsection{CAS 20-22-29: Bucket}

One unopened and intact 19-L (5-gal) bucket of lithium grease was located at the site. One soil sample (202229-1) was collected from beneath the bucket and was analyzed for VOCs, SVOCs, RCRA metals, TPH, PCBs, and gamma emitters. The analytical results indicated that concentrations in the soil were either below the appropriate action level or laboratory method detection levels. Therefore, no soil was required to be removed from the site. Based on the Material Safety Data Sheet (MSDS) information for this material, the bucket of grease was determined to be hydrocarbon waste. The analytical data summary for the sample is included in Appendix A. 


\subsection{SITE CLOSURE ACTIVITIES}

Appendix B provides the Housekeeping Site Closure Verification Forms including site information and before and after photographs.

\subsubsection{CAS 04-22-04: Drums}

The drums originally identified in Appendix II of the FFACO (1996) were not present during the site visit. The drums were originally found inside the bottom of the U-4h crater from the 1977 "Scantling" test (DOE/NV, 2000a). Site closure was documented with photographs and field notes. No further action is required at this site.

\subsubsection{CAS 12-22-20: Drum}

The drum was disposed of as sanitary waste in the Area $9 \mathrm{U}-10 \mathrm{c}$ Landfill as an empty container as defined by Title 40 CFR Part 261.7 (CFR 2002a). Based on the results of the characterization sampling (Table 1) no soil was required to be removed from the site. Site closure was documented with photographs and field notes. No further action is required at this site.

\subsubsection{CAS 12-30-16: Manhole for Gas/Air Line}

The manhole identified in the FFACO (1996) is part of the currently inactive 12-2 water transmission line running between the Area 12 Camp and the former Area 2 Camp. The manhole contains an air/vacuum release valve. The manhole and materials inside are not waste material and may not be removed as long as the 12-2 Line is in place. For this reason, no action was taken to close this site; a FFACO modification to remove CAS 12-30-16 from CAU 355 and place it in CAU 5000 has been prepared, submitted, and is pending approval. Documentation of the status of the water line is included in

Appendix D.

\subsubsection{CAS 16-22-29: Drum}

The drum originally identified in Appendix II of the FFACO (1996) was not present during the site visit. The drum was originally found near the U-16a portal. Site closure was documented with photographs and field notes. No further action is required at this site.

\subsubsection{CAS 18-22-24: Bottles - Lithium Bromide}

The bottles of lithium bromide originally identified in Appendix II of the FFACO (1996) were not present at the time of the site visit. The empty drill collar compound bucket was disposed of at the Area 9 U-10c Landfill as an empty container according to Title 40 CFR Part 261.7 (CFR 2002a). Based on the results of the characterization sampling (Table 1) soil was not required to be removed from the site. Site closure was documented with photographs and field notes. No further action is required at this site. 


\subsubsection{CAS 19-22-02: Drums (3)}

The drums originally identified in Appendix II of the FFACO (1996) were not present during the site visit. The drums were originally found near a mud pit associated with the U-19ar crater from the 1986 "Cybar" test (DOE/NV, 2000a). Site closure was documented with photographs and field notes. No further action is required at this site.

\subsubsection{CAS 19-22-04: Drums (3)}

The drums originally identified in Appendix II of the FFACO (1996) were not present during the site visit. The drums were originally found near the postshot cellars associated with the U-19ay crater from the 1989 "Amarillo" test (DOE/NV, 2000a). However, approximately 50 short steel rods were located at the site and were removed and transported to the Area 9 U-10c Landfill for disposal, as a best management practice. Site closure was documented with photographs and field notes. No further action is required at this site.

\subsubsection{CAS 19-22-31: Can}

All three empty cans were removed from the site as a best management practice and were disposed of as ordinary waste in the U-10c Landfill as empty containers according to Title 40 CFR Part 261.7 (CFR 2002a). Additionally, approximately 0.028 cubic meters $\left(\mathrm{m}^{3}\right.$ ) (one cubic foot $\left[\mathrm{ft}^{3}\right]$ ) of soil was removed and disposed of at the Area 6 Hydrocarbon Landfill. Two verification samples collected from beneath the excavated soil were analyzed for TPH. The verification sample results were below the $100 \mathrm{mg} / \mathrm{kg}$ State of Nevada action level (NAC, 2003). Analytical results for the verification samples are presented in Table 2. Site closure was documented with photographs and field notes. No further action is required at this site.

\subsubsection{CAS 19-22-32: Can; Debris}

Observations made during the site visit determined that the cans and debris originally identified in Appendix II of the FFACO (1996) had been recently moved from their original location during road improvements. The debris included one empty 19-L (5-gal) bucket, a broken chair, a broken wooden box, and several pieces of scrap metal. The original location could not be precisely determined. Therefore, the empty can and debris were removed and transported to the Area 9 U-10c Landfill for disposal and no sampling was performed. Site closure was documented with photographs and field notes. No further action is required at this site.

\subsubsection{CAS 20-22-03: Drums}

The drums originally identified in Appendix $\Pi$ of the FFACO (1996) were not present during the site visit. The drums were originally found near the U-20p ground zero from the 1975 "Stilton" test (DOE/NV, 2000a). Other wood and scrap metal debris located at the site but could not be removed because the potential crater area is inaccessible to vehicles and due to worker safety. Site closure was documented with photographs and field notes. No further action is required at this site. 
TABLE 2 - SUMMARY OF ANALYTICAL RESULTS FOR VERIFICATION SOIL SAMPLES

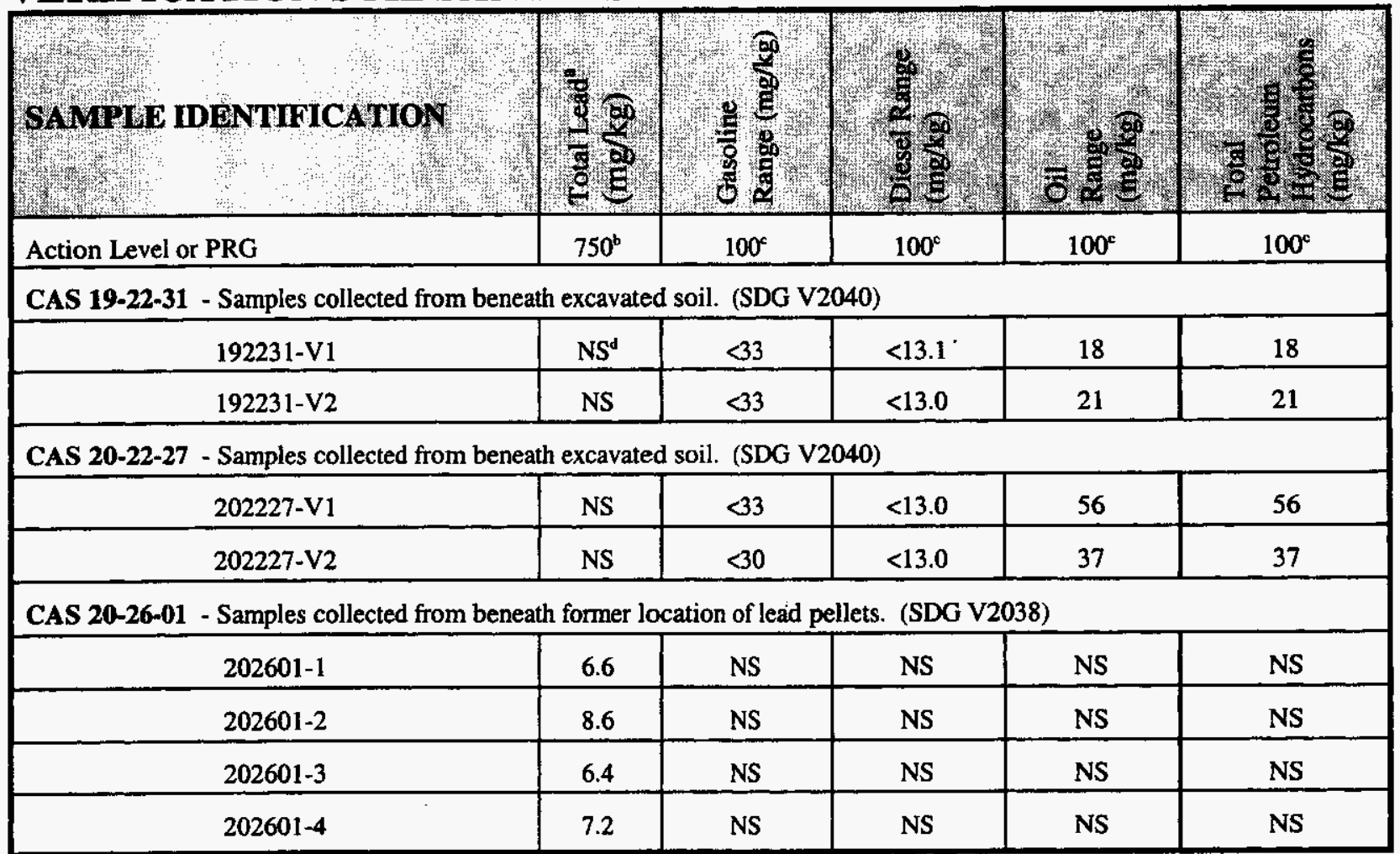

Note: All samples analyzed by EPA Method SW-846 6010 and 8015 modified (EPA, 1996). See analytical reports in Appendix A for detection limits.

$\cdot \mathrm{mg} / \mathrm{kg}=$ Milligram(s) per kilogram.

- U.S. EPA Region IX Preliminary Remediation Goals for industrial soil (EPA, 2002).

'State of Nevada Remediation Action Levels for total petroleum hydrocarbons (NAC, 2003).

¿NS = Not Sampled

\subsubsection{CAS 20-22-10: Drum}

The drum originally identified in Appendix II of the FFACO (1996) was not present during the site visit. The drum was originally found near the U-20a crater from the 1965 and 1966 "Buteo" and "Duryea" tests (DOE/NV, 2000a). Site closure was documented with photographs and field notes. No further action is required at this site.

\subsubsection{CAS 20-22-20: Drum}

The drum originally identified in Appendix II of the FFACO (1996) was not present during the site visit. The drum was originally found near the U-20az crater from the 1989 "Barnwell" test (DOE/NV, 2000a). Site closure was documented with photographs and field notes. No further action is required at this site.

\subsubsection{CAS 20-22-27: Bucket}

The empty bucket was disposed of as sanitary waste at the Area 9 U-10c Landfill as an empty container according to Title 40 CFR Part 261.7 (CFR 2002a). The additional drum found at the site was also disposed of at the U-10c Landfill as a best management practice. Additionally, 
approximately $0.028 \mathrm{~m}^{3}\left(1 \mathrm{ft}^{3}\right)$ of soil was removed for disposal at the Area 6 Hydrocarbon Landfill. Two verification samples collected from beneath the excavated soil at the former location of the drum were analyzed for TPH. The verification sample results were below the $100 \mathrm{mg} / \mathrm{kg}$ State of Nevada action level (NAC, 2003). Analytical results for the verification samples are presented in Table 2. Site closure was documented with photographs and field notes. No further action is required at this site.

\subsubsection{CAS 20-22-28: Drums}

The three 113.5-L (30-gal) drums and their contents were disposed of at the Area 9 U-10c Landfill as ordinary waste. Based on the results of the characterization sampling (Table 1) no soil was required to be removed from the site. Site closure was documented with photographs and field notes. No further action is required at this site.

\subsubsection{CAS 20-22-29: Bucket}

The intact bucket of grease was removed from the area west of the U-20aa crater from the 1976 "Colby" test (DOE/NV, 2000a) and disposed of at the Area 6 Hydrocarbon Landfill. Based on the results of the characterization sampling (Table 1) no soil was required to be removed from the site. Site closure was documented with photographs and field notes. No further action is required at this site.

\subsubsection{CAS 20-26-01: Lead Pellets}

The lead pellets and lead impacted soil were removed from the area directly north of the U20ar crater formed from the 1988 "Kernville" test (DOE/NV, 2000a). The majority of the lead pellets were able to be segregated from the soil. One 208-L (55-gal) drum of segregated lead pellets was sent to the Excess Yard for salvage on August 7, 2003. One 208-L (55-gal) drum of lead impacted soil was staged in a Satellite Accumulation Area (SAA) and disposed of at an approved off-site Treatment and Storage Disposal facility as hazardous waste on August 26, 2003. Four verification samples were collected from the soil beneath the remediated area and were analyzed for total lead. Results exhibited total lead concentrations below the EPA Region 9 PRG for industrial soils (EPA, 2002). Analytical results for the verification samples are presented in Table 2. Site closure was documented with photographs and field notes. No further action is required at this site. 


\subsection{WASTE DISPOSITION}

Waste generated during the closure of CAU 523: Housekeeping Waste was disposed of as follows:

- CAS 12-22-20: One empty 208-L (55-gal) drum was disposed of as ordinary waste in the Area 9 U-10c Landfill.

- CAS 18-22-24: One empty bucket was disposed of as ordinary waste in the Area 9 U-10c Landfill.

- CAS 19-22-04: Approximately 50 steel rods were disposed of as ordinary waste in the Area 9 U-10c Landfill.

- CAS 19-22-31: Three empty cans were disposed of as ordinary waste in the Area 9 $\mathrm{U}-10 \mathrm{c}$ Landfill. In addition, $0.028 \mathrm{~m}^{3}\left(1 \mathrm{ft}^{3}\right)$ of soil was disposed of as hydrocarbon waste in the Area 6 Hydrocarbon Landfill.

- CAS 19-22-32: One empty bucket and other debris were disposed of as ordinary waste in the Area 9 U-10c Landfill.

- CAS 20-22-27: One empty 19-L (5-gal) bucket and one empty 208-L (55-gal) drum were disposed of as ordinary waste in the Area $9 \mathrm{U}-10 \mathrm{c}$ Landfill. In addition, $0.028 \mathrm{~m}^{3}\left(1 \mathrm{ft}^{3}\right)$ of soil was disposed of as hydrocarbon waste in the Area 6 Hydrocarbon Landfill.

- CAS 20-22-28: Three empty 113.5-L (30-gal) drums were disposed of as ordinary waste in the Area $9 \mathrm{U}-10 \mathrm{c}$ Landfill.

- CAS 20-22-29: One intact bucket of grease was disposed of as hydrocarbon waste in the Area 6 Hydrocarbon Landfill.

- CAS 20-26-01: One 208-L (55-gal) drum of segregated lead pellets was sent to the Excess Yard for salvage on August 7, 2003. One 208-L (55-gal) drum of lead pellets and soil was sent to an approved off-site facility for disposal as hazardous waste. 
Revision: 0

Date: November 2003

THIS PAGE INTENTIONALLY LEFT BLANK 


\subsection{CLOSURE VERIFICATION}

The following three CASs required verification soil sampling:

- CAS 19-22-31

- $\quad$ CAS 20-22-27

- $\quad$ CAS 20-26-01

Verification samples were collected from a total of eight locations after the removal of debris and the removal of small amounts of soil determined by waste characterization sample analytical results. The samples were collected with clean disposable plastic scoops and placed in labeled sample containers secured with custody seals. The sample containers were placed on ice in a cooler and maintained at less than 4 degrees Celsius. They were transported under chain of custody to the Environmental Technical Services group in Mercury, Nevada and shipped to an off-site laboratory for analysis. Samples collected from the three CASs requiring verification sampling were analyzed for the following:

- $\quad$ CAS 19-22-31

- CAS 20-22-27

TPH full scan

- CAS 20-26-01

TPH full scan

Total Lead

The analytical results verified that COCs remaining in the ground at the three CASs were below EPA Region 9 PRGs for industrial soil (EPA, 2002) or the State of Nevada action level for TPH (NAC, 2003). The analytical results for the verification samples are summarized in Table 2. Copies of the analytical reports are found in Appendix A. 


\subsection{SUMMARY AND RECOMMENDATIONS}

\subsection{SUMMARY}

The following site closure activities were performed for the 16 CASs in CAU 523:

- All containers (drums, cans, scrap metal, etc.) were disposed of at the Area 9 U-10c Landfill.

- $\quad$ All hydrocarbon waste (grease bucket) and hydrocarbon impacted soil were disposed of at the Area 6 Hydrocarbon Landfill.

- All salvageable materials (segregated lead pellets) were presented to the NTS excess yard for salvage.

- The 208-L (55-gal) drum of lead pellets/soil mixture was placed in an SAA and was disposed of as hazardous waste.

\subsection{RECOMMENDATIONS}

Since the closure activities for CAU 523 have been completed following the Nevada Division of Environmental Protection (NDEP)-approved Sectored Clean-up Work Plan for Housekeeping Category Waste Sites (NNSA/NSO, 2003) as documented in this report, NNSA/NSO requests the following:

1. A Notice of Completion provided by the NDEP to the NNSA/NSO for the closure of all 15 CASs in CAU 523:

- CAS 04-22-04

- CAS 12-22-20

- CAS 16-22-29

- $\quad$ CAS 18-22-24

- CAS 19-22-02

- CAS 19-22-04

- CAS 19-22-31

- CAS 19-22-32

- CAS 20-22-03

- CAS 20-22-10

- CAS 20-22-20

- CAS 20-22-27

- CAS 20-22-28

- CAS 20-22-29

- CAS 20-26-01

Note: A FFACO modification requesting that CAS 12-30-16 be removed from CAU 355 and placed in CAU 5000 has been prepared, submitted, and is pending approval.

2. CAU 523 moved from Appendix III to Appendix IV of the FFACO Closed Corrective Action Units (FFACO, 1996). 


\section{Revision: 0}

Date: November 2003

THIS PAGE INTENTIONALLY LEFT BLANK 


\subsection{REFERENCES}

Bechtel Nevada. 2002. Generic Field Management Plan for Housekeeping Category Waste Sites, Las Vegas, NV.

Bechtel Nevada. 2003. Site-Specific Health and Safety Plan for Corrective Action Units 176. $346,350,351,352, \& 523$ Housekeeping Sites, Nevada Test Site, Nevada, Rev. 1, Las Vegas, NV.

BN, see Bechtel Nevada.

CFR, see Code of Federal Regulations.

Code of Federal Regulations. 2002a. Title 40, Part 261, "Identification and Listing of Hazardous Waste." Washington D.C.

Code of Federal Regulation. 2002b. Title 40, Part 761, "Polychlorinated biphenyls (PCBs)

Manufacturing, Processing, Distribution in Commerce, and Use Prohibitions."

Washington D.C.

DOE/NV, see U.S. Department of Energy, Nevada Operations Office.

EPA, see U.S. Environmental Protection Agency.

FFACO, see Federal Facility Agreement and Consent Order.

Federal Facility Agreement and Consent Order. 1996 (as amended). Agreed to by the State of Nevada, U.S. Department of Energy, and U.S. Department of Defense.

NAC, see Nevada Administrative Code.

Nevada Administrative Code. 2003. NAC 445A.2272, Contamination of Soil: Establishment of Action Levels. Carson City, NV.

NNSA/NSO, see U.S. Department of Energy, National Nuclear Security Administration Nevada Site Office.

U.S. Department of Energy, Nevada Operations Office. 2000a. United States Nuclear Tests, July 1945 through September 1992. DOE/NV--209 (Rev. 15). Las Vegas, NV.

U.S. Department of Energy, Nevada Operations Office, 2000b. Revision 4. NV/YMP Radiological Control Manual, DOE/NV/11718-079, Las Vegas, Nevada.

U.S. Department of Energy, Nevada Site Office. 2003. Sectored Clean-up Work Plan for Housekeeping Category Waste Sites, DOE/NV--579-REV-2, Las Vegas, NV. 
U.S. Environmental Protection Agency. 1996. Test Methods for Evaluating Solid Waste, Physical/Chemical Methods, EPA Publication SW-846, Third Edition, Washington D.C.

U.S. Environmental Protection Agency. 2002. Region IX Preliminary Remediation Goals (PRGs), San Francisco, CA. 
Closure Report - CAU 523

Section: Appendix A

Revision: 0

Date: November 2003

\section{APPENDIX A}

\section{SAMPLE ANALYTICAL RESULTS}


Closure Report - CAU 523

Section: Appendix A

Revision: 0

Date: November 2003

\section{THIS PAGE INTENTIONALLY LEFT BLANK}




\section{APPENDIX A TABLE OF CONTENTS - SAMPLE ANALYTICAL RESULTS BY SAMPLE DELIVERY GROUP}

Analytical results for the samples collected at the indicated Corrective Action Site (CAS) for Corrective Action Unit 523 are presented in this Appendix. The analytical results are grouped by Sample Delivery Group(s), which are arranged in numerical order in this Appendix. Analytical results for the specific CAS can be found by consulting the indicated Sample Delivery Group(s).

CAS Sample Delivery Group(s)

12-22-20 V2004

18-22-24 V1978 and V1979

19-22-31 V1978, V1979, and V2040

20-22-27 V1978, V1979, 2029, and 2040

20-22-28 V1978 and V1979

20-22-29 V1978 and V1979

20-26-01 V2038 
Date: November 2003

\section{THIS PAGE INTENTIONALLY LEFT BLANK}




\section{SAMPLE DELIVERY GROUP}

\section{V1978}


Date: November 2003

\section{THIS PAGE INTENTIONALLY LEFT BLANK}




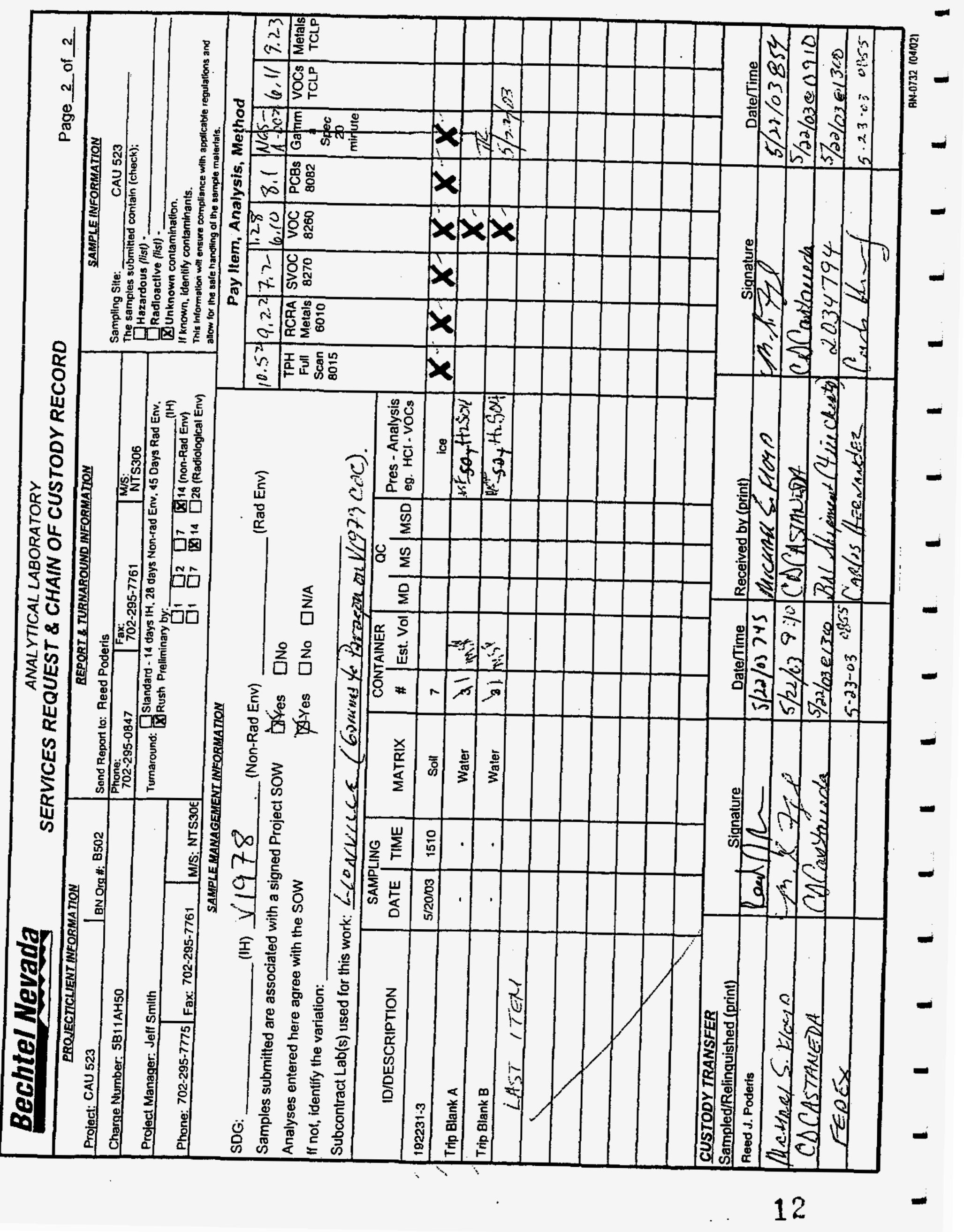


1

INORGANIC ANALYSES DATA SHEET
Lab Name: LIONVILLE_LABORATORY

Lab Code: IVII

Matrix (soil/water): SOIL_

Level (low/med): LOW

$\%$ Solids :
Contract: 60052

SAS NO.:

SDG No : 2224-1

Lab Sample ID: 0305L484-001

Date Received: 05/23/03

Concentration Units (ug/L or $\mathrm{mg} / \mathrm{kg}$ dry weight) : MG/KG

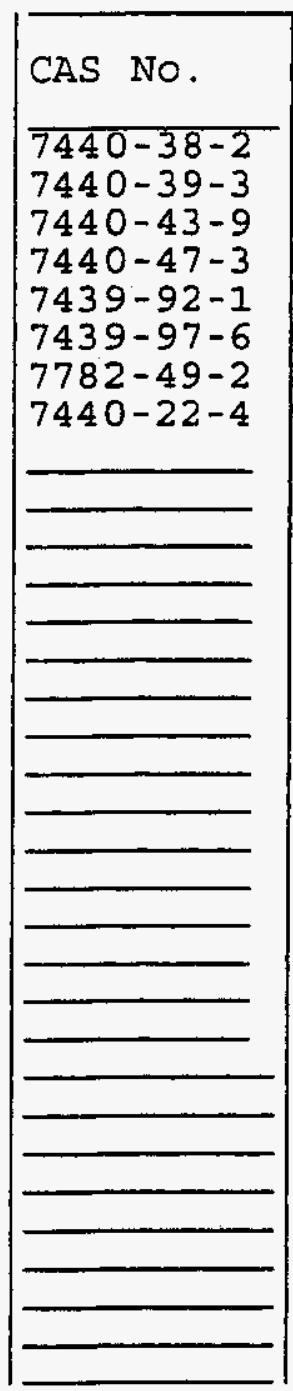

Color Before: Color After:

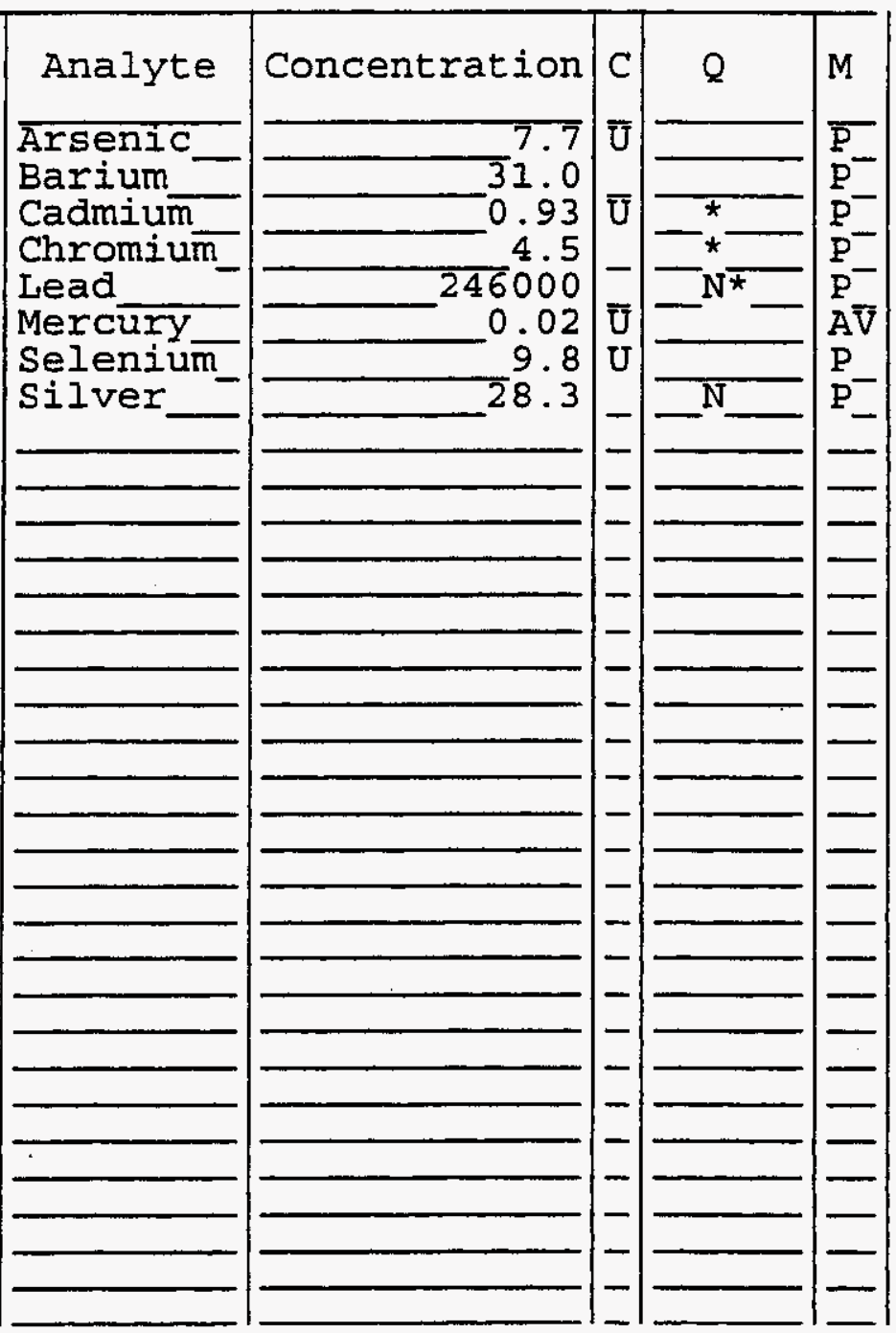

Clarity Before: Clarity After:
Texture:

Artifacts :

\section{Comments :}

\section{82224-1}


1

INORGANIC ANALYSES DATA SHEET
EPA SAMPLE NO.

SDG NO.: 2224-1

Lab Name: LIONVILLE_LABORATORY

Lab Code: IVLI

Matrix (soil/water): SOIL

Level (low/med): LOW

$\div$ Solids :
Contract: 60052

SAS No.:

Lab Sample ID: 0305L484-002

Date Received: 05/23/03

Concentration Units (ug/L or $\mathrm{mg} / \mathrm{kg}$ dry weight): MG/KG

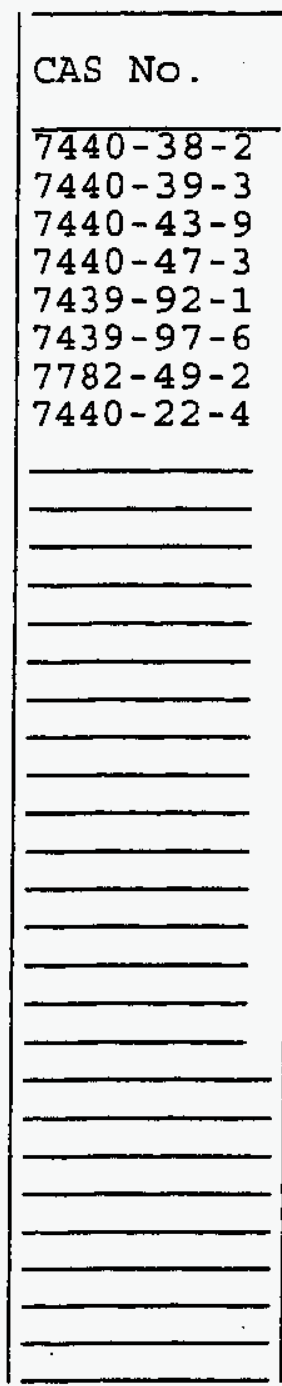

Color Before:

Color After:

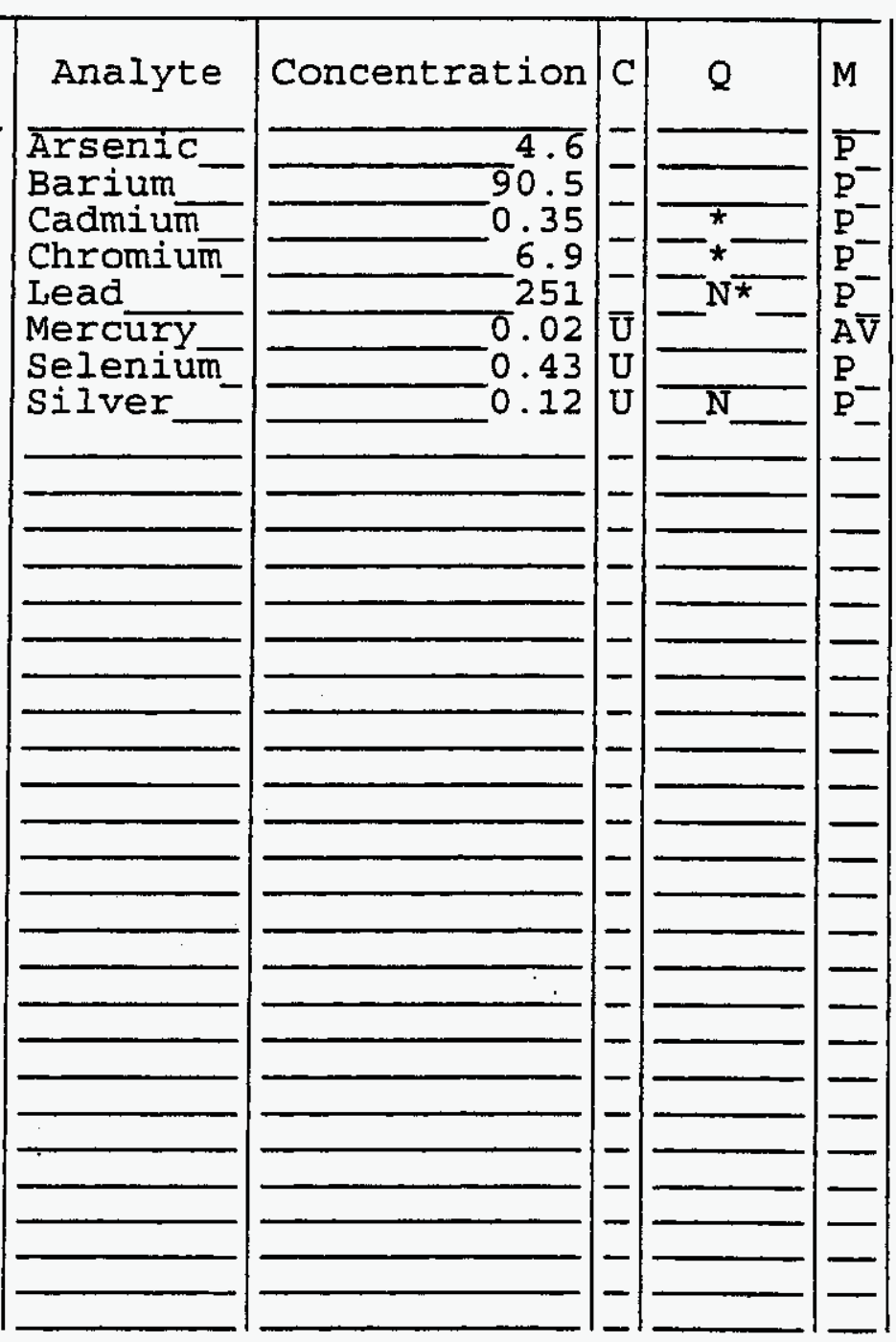

Clarity Before:

Clarity After:
Texture:

Artifacts :

Comments :

182224-2 
U.S. EPA

\section{1 \\ INORGANIC ANALY̌SES DATA SHEET}

EPA SAMPLE NO .

$$
2227-1
$$

Lab Name: LIONVILLE_LABORATORY Lab Code: IVLI_ Case No.: VI978

Matrix (soil/water): SOIL_ Level (low/med) : LOW : Solids :

Contract: 60052

SAS No.:

L $94 \overline{3}$

Lab Sample ID: 0305L484-007

Date Received: 05/23/03

SDG NO.: 2224-1

Concentration Units (ug/L or $\mathrm{mg} / \mathrm{kg}$ dry weight): MG/KG

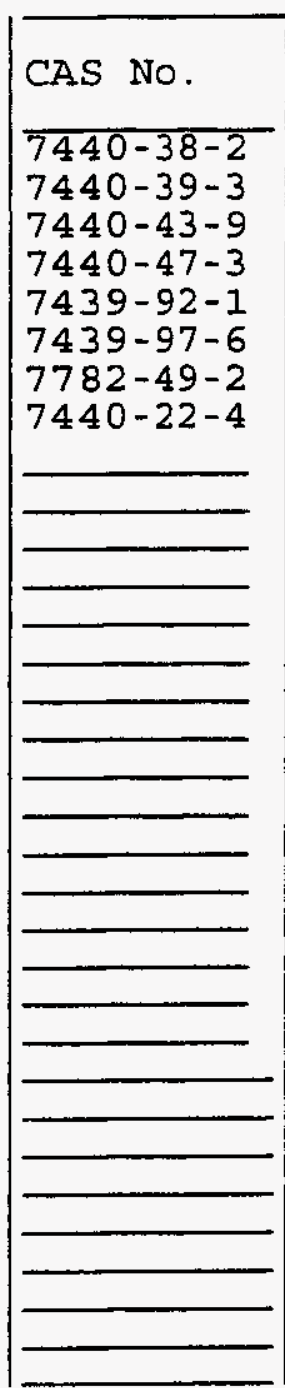

Color Before:

Color After:

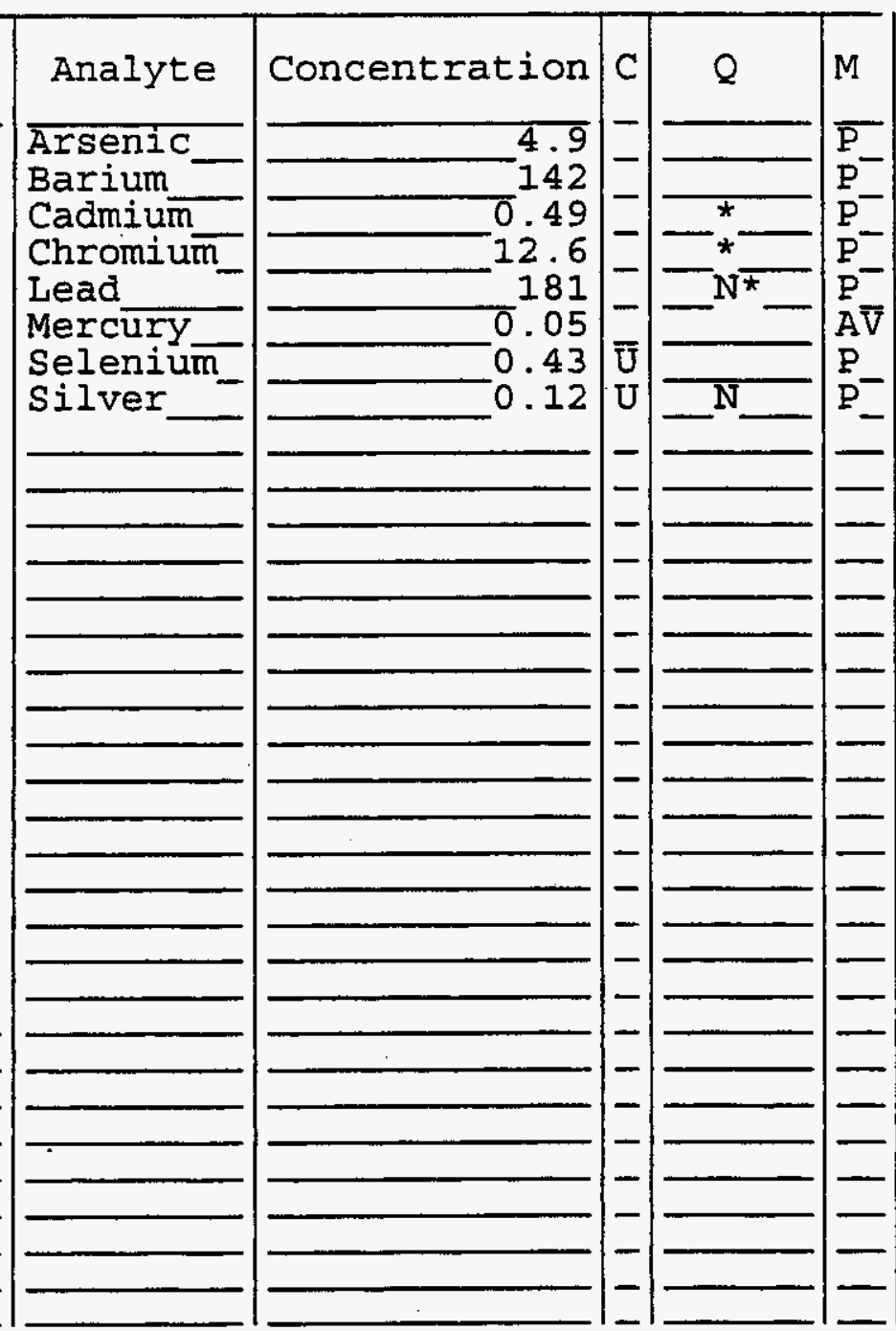

Clarity Before: Clarity After:
Texture: Artifacts:

\section{Comments :}

$202227-1$ 
EPA SAMPLE NO.

$$
\text { 2227-2 }
$$

Contract: 60052

Lab Name: LIONVILLE_LABORATORY SAS No.:

Case No.: V1978

Lab Code: LVII

Matrix (soil/water): SOIL_
Level (low/med):

o Solids:
Lab Sample ID: 0305L484-008

Date Received: 05/23/03

Concentration Units (ug/L or $\mathrm{mg} / \mathrm{kg}$ dry weight): MG/KG

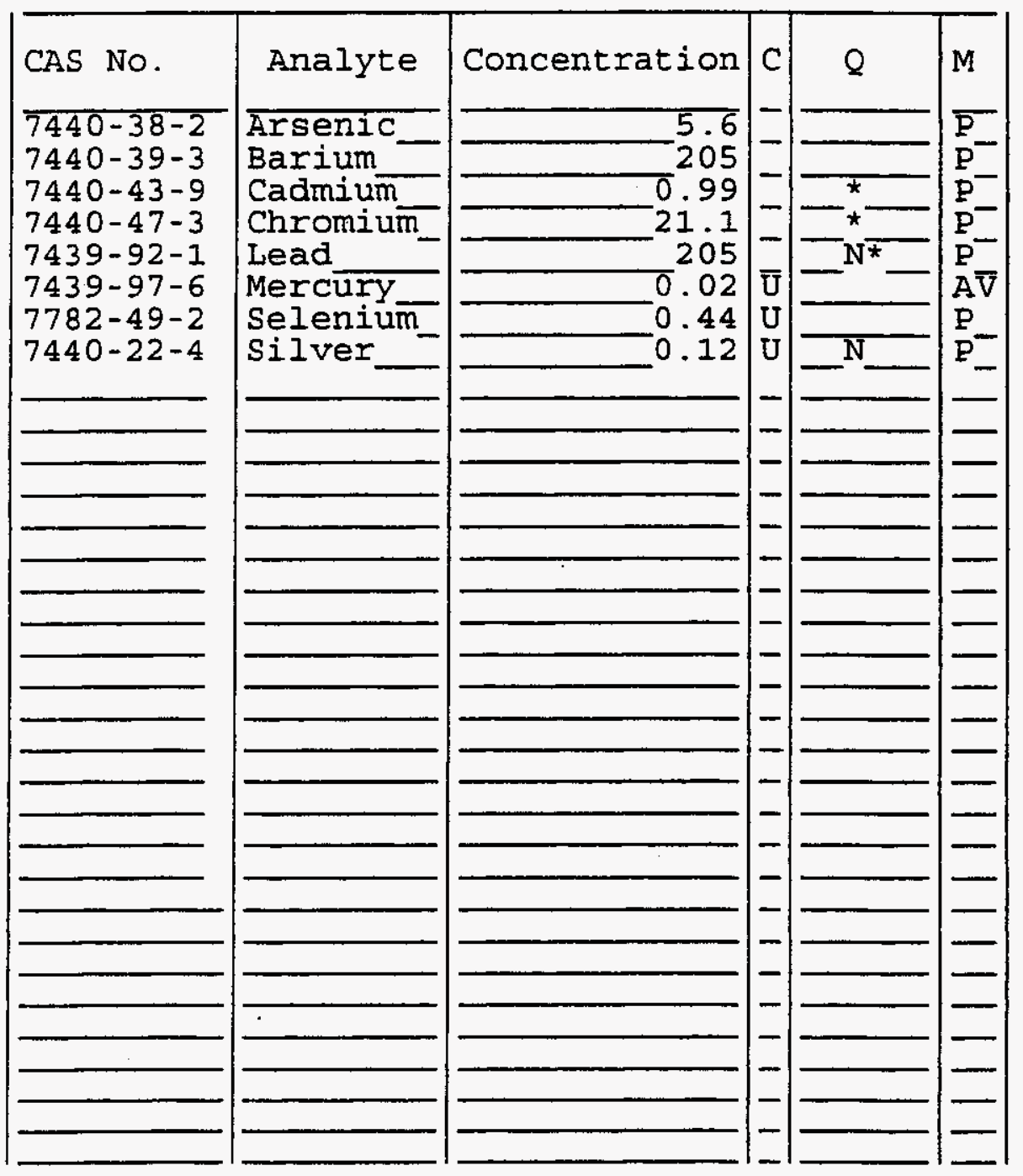

Color Before: Color After:
Clarity Before:

Clarity After:
Texture:

Artifacts:

\section{Comments :}

\section{7-2}


1 INORGANIC ANALYSES DATA SHEET
EPA SAMPLE NO.

$2227-3$

Contract: 60052

SAS No.:

SDG NO.: 2224-1

Lab Name: LIONVILLE_LABORATORY

Case No.: VI978

Matrix (soil/water): SoII_

Level (low/med): LOW

\% Solids :

Date Received: 05/23/03

Concentration Units (ug/L or $\mathrm{mg} / \mathrm{kg}$ dry weight): MG/KG

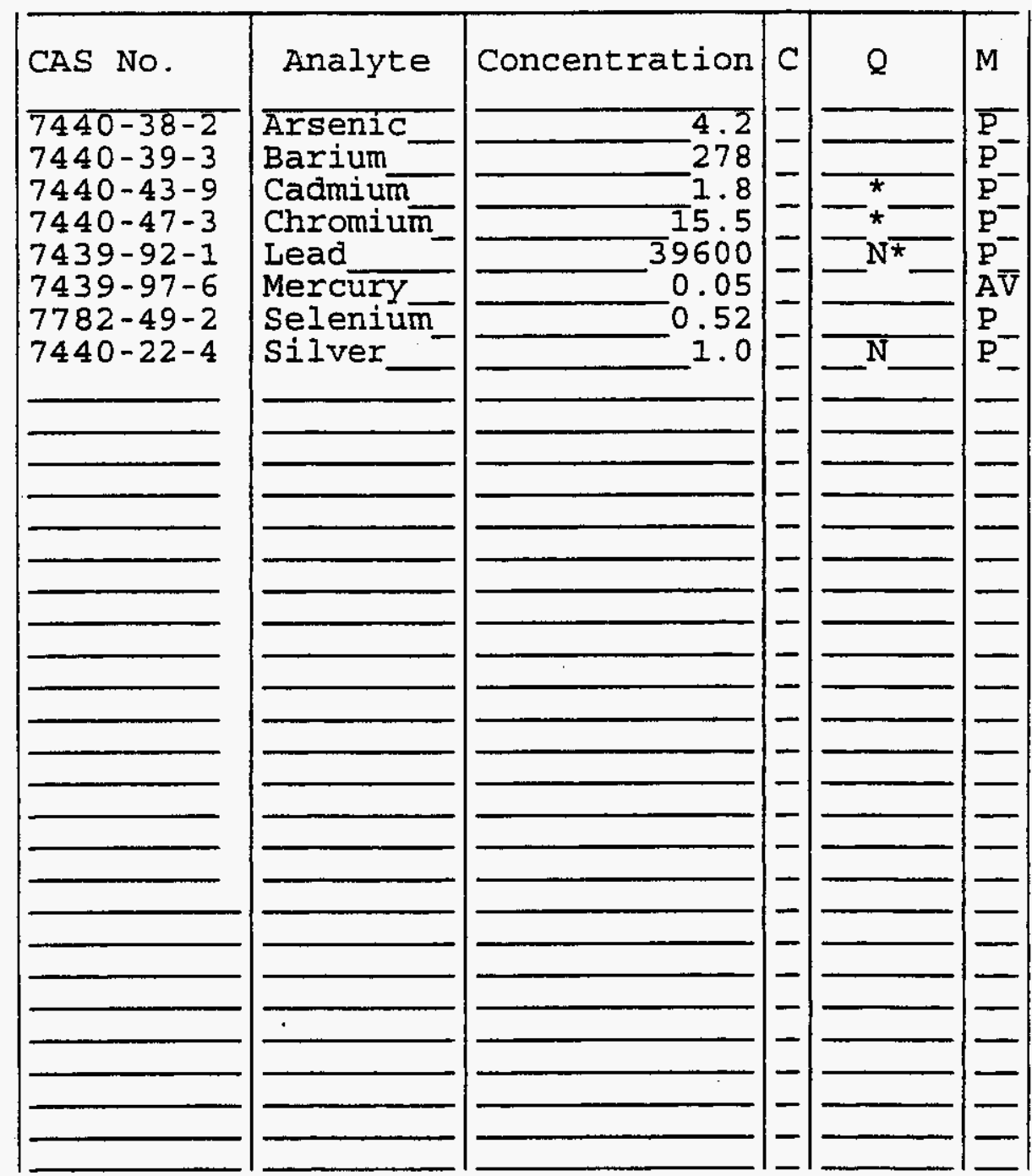

Color Before:

Color After:
Clarity Before:

Clarity After:
Texture:

Artifacts:

\section{Comments :}

202227-3 
1

INORGANIC ANALYSES DATA SHEET
EPA SAMPLE NO.

2228-2

Contract: 60052

Lab Name: LIONVILLE_LABORATORY

Lab code: LVLI

Case No.: V1978 SAS No.:

Matrix (soil/water): SoIL_

Level (low/med):

\% Solids :
Lab Sample ID: 0305L484-004

Date Received: 05/23/03

Concentration Units (ug/L or $\mathrm{mg} / \mathrm{kg}$ dry weight): MG/KG

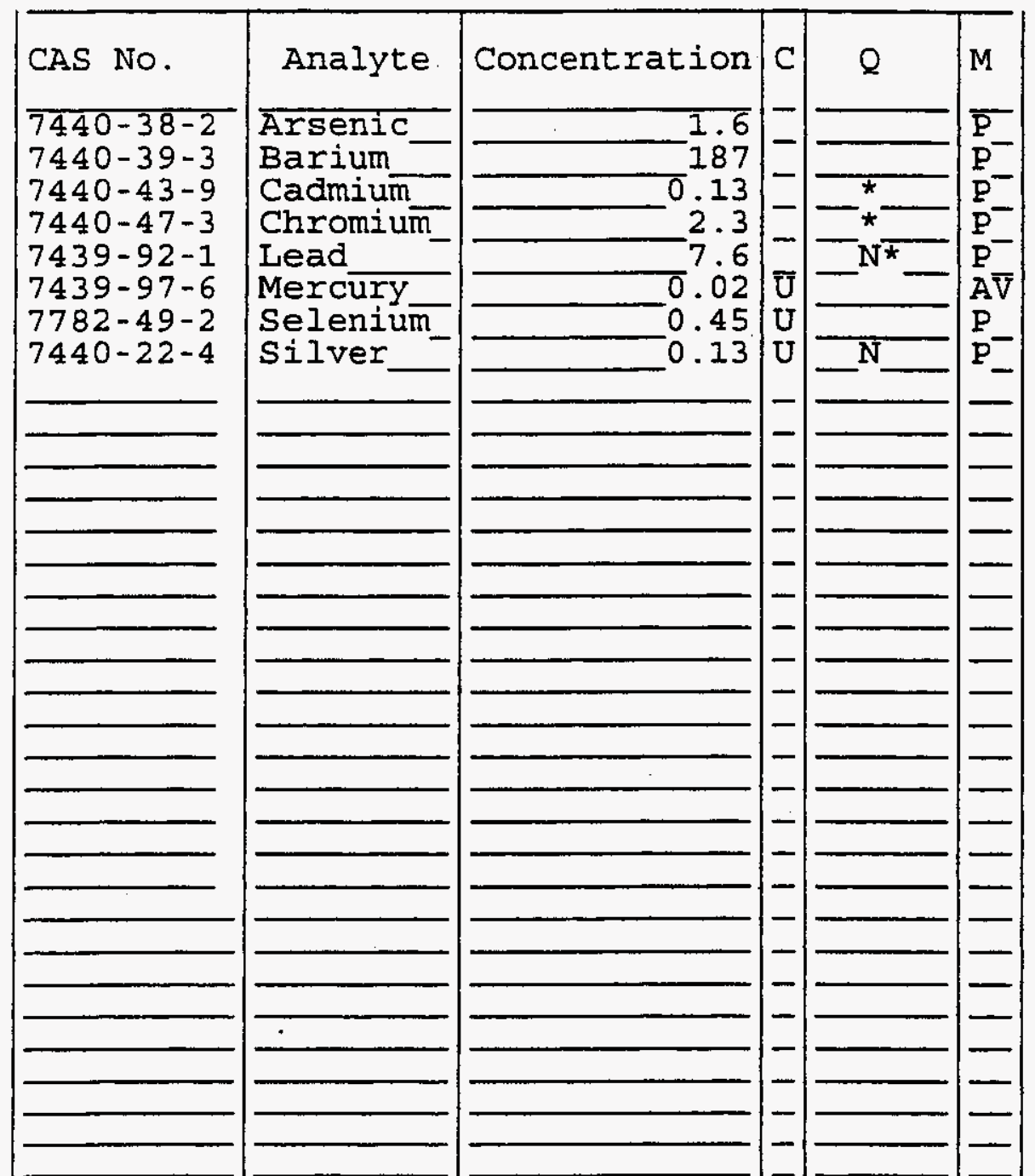

Color Before:

Clarity Before:

Clarity After:

Texture:

Artifacts : 
1

INORGANIC ANALYSES DATA SHEET
Lab Name: LIONVILLE ILABORATORY

Lab Code: LVII

Matrix (soil/water): SoIL

Level (low/med) : LOW.

\% Solids: $\quad-91 . \overline{8}$
EPA SAMPLE NO.

$2228-3$

Contract : 60052

SAS No.:

Lab Sampl SDG No.: 2224-1

Date Received: 05/23/03

Concentration Units (ug/L or $\mathrm{mg} / \mathrm{kg}$ dry weight): MG/KG

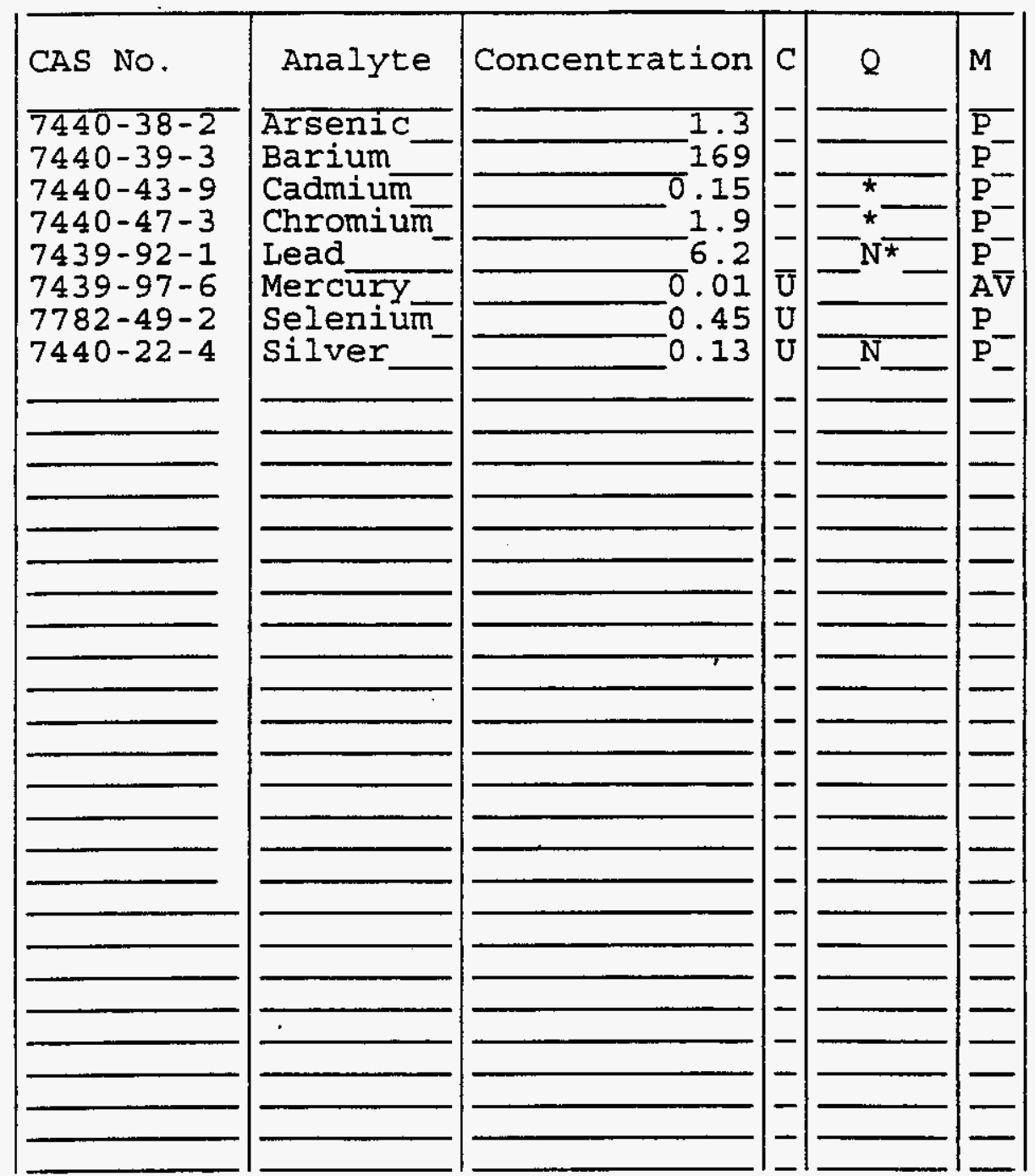

Color Before:

Clarity Before:

Clarity After:

Texture:

Artifacts:

Comments :

202228-3 
1 INORGANIC ANALYSES DATA SHEET
EPA SAMPLE NO.

$$
\text { 2229-1 }
$$

Contract: 60052

SAS No.:

Lab Sample ID: 0305L484-006

Matrix (soil/water): SoIL

Level (low/med) :

$\div$ Solids:

Date Received: 05/23/03

LOW

Concentration Units (ug/L or $\mathrm{mg} / \mathrm{kg}$ dry weight) : MG/KG

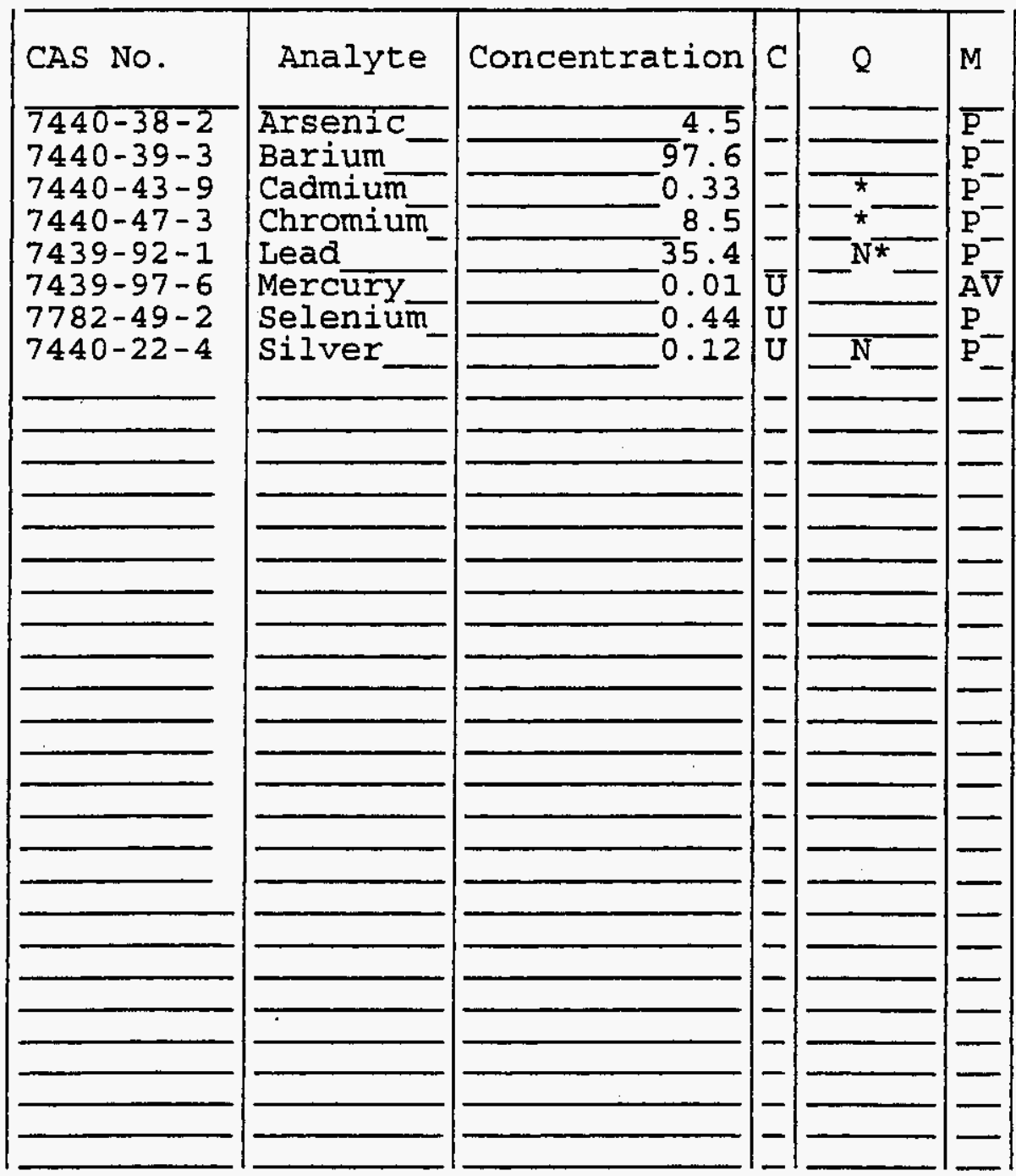

Color Before:

Color After:
Clarity Before:

Clarity After:
Texture:

Artifacts :

Comments :

202229-1 
U.S. EPA

1

INORGANIC ANALYSES DATA SHEET
EPA SAMPLE NO.

$2231-1$

Lab Name: LIONVILLE LABORATORY

Lab Code: LVLI

Matrix (soil/water): SOIL

Level (low/med): LOW

\% Solids :

Contract: 60052
SAS No.:

SDG No.: 2224-1

Lab Sample ID : 0305L484-010

Date Received: 05/23/03

Concentration Units (ug/L or $\mathrm{mg} / \mathrm{kg}$ dry weight): MG/KG

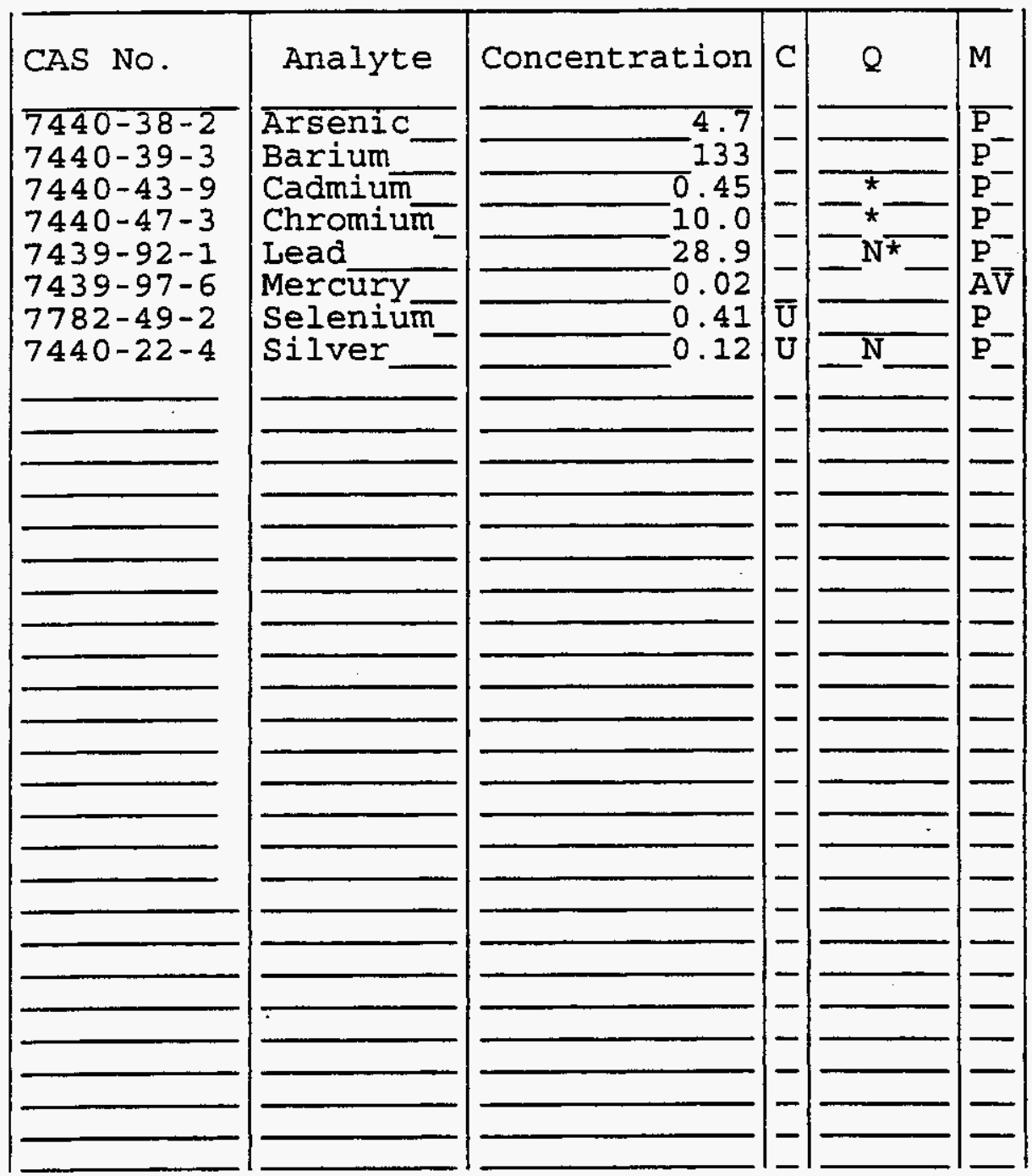

Color Before:

Clarity Before:

Clarity After:

Texture:

Artifacts:

\section{Comments :}

192231-1 
1

INORGANIC ANALYSES DATA SHEET
Lab Name: LIONVILLE_LABORATORY

Lab Code: LVII

Matrix (soil/water): SOIL_

Level (low/med): \% Solids : LOW 98.7
Contract: 60052

SAS No. :

Iab Sample ID: $03051484-011$
EPA SAMPLE NO.

2231-3

Date Received: 05/23/03

Concentration Units (ug/L or $\mathrm{mg} / \mathrm{kg}$ dry weight): MG/KG

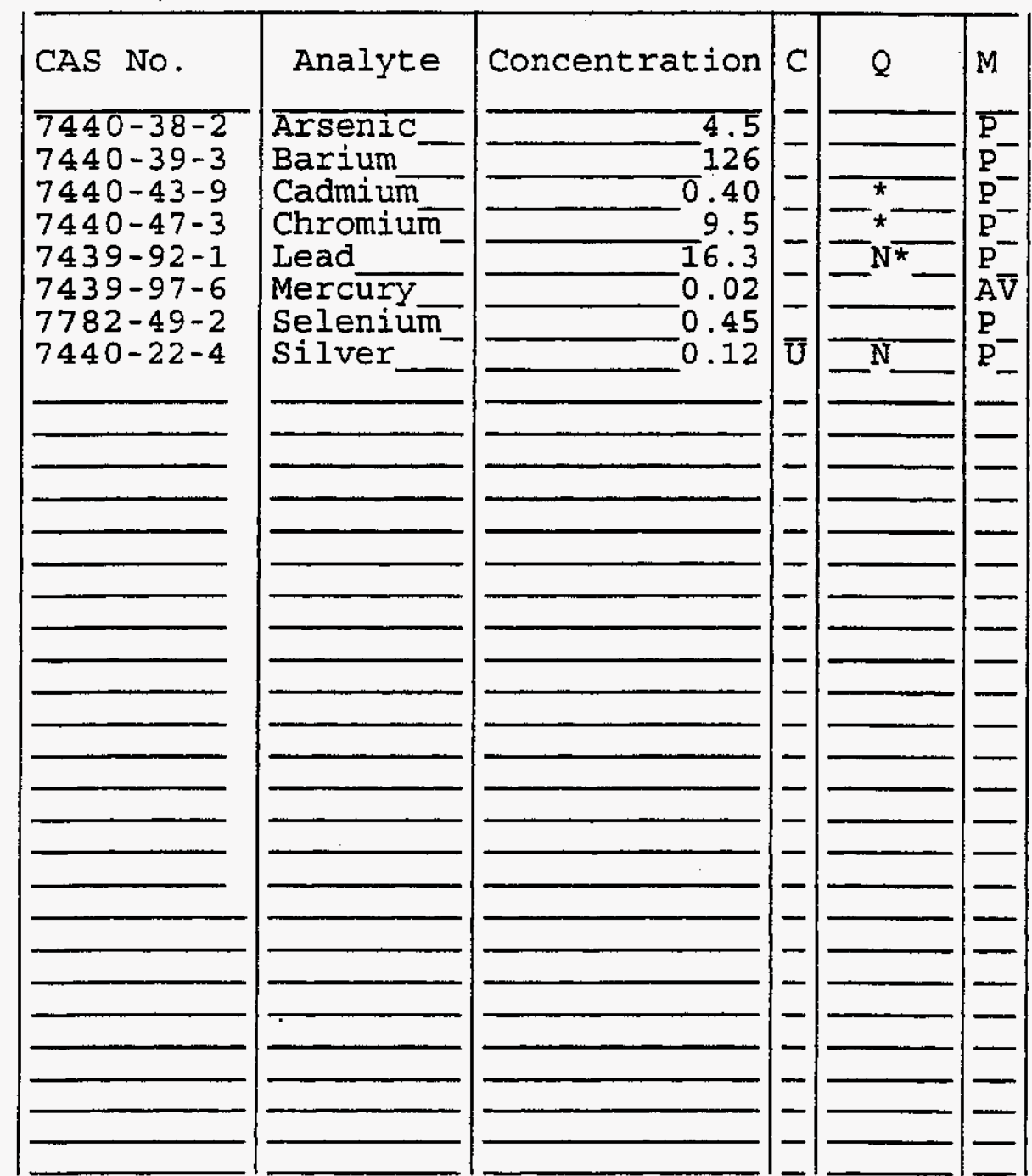

Color Before:

Color After:
Clarity Before: Clarity After:
Texture:

Artifacts :

\section{Comments :}

192231-3 


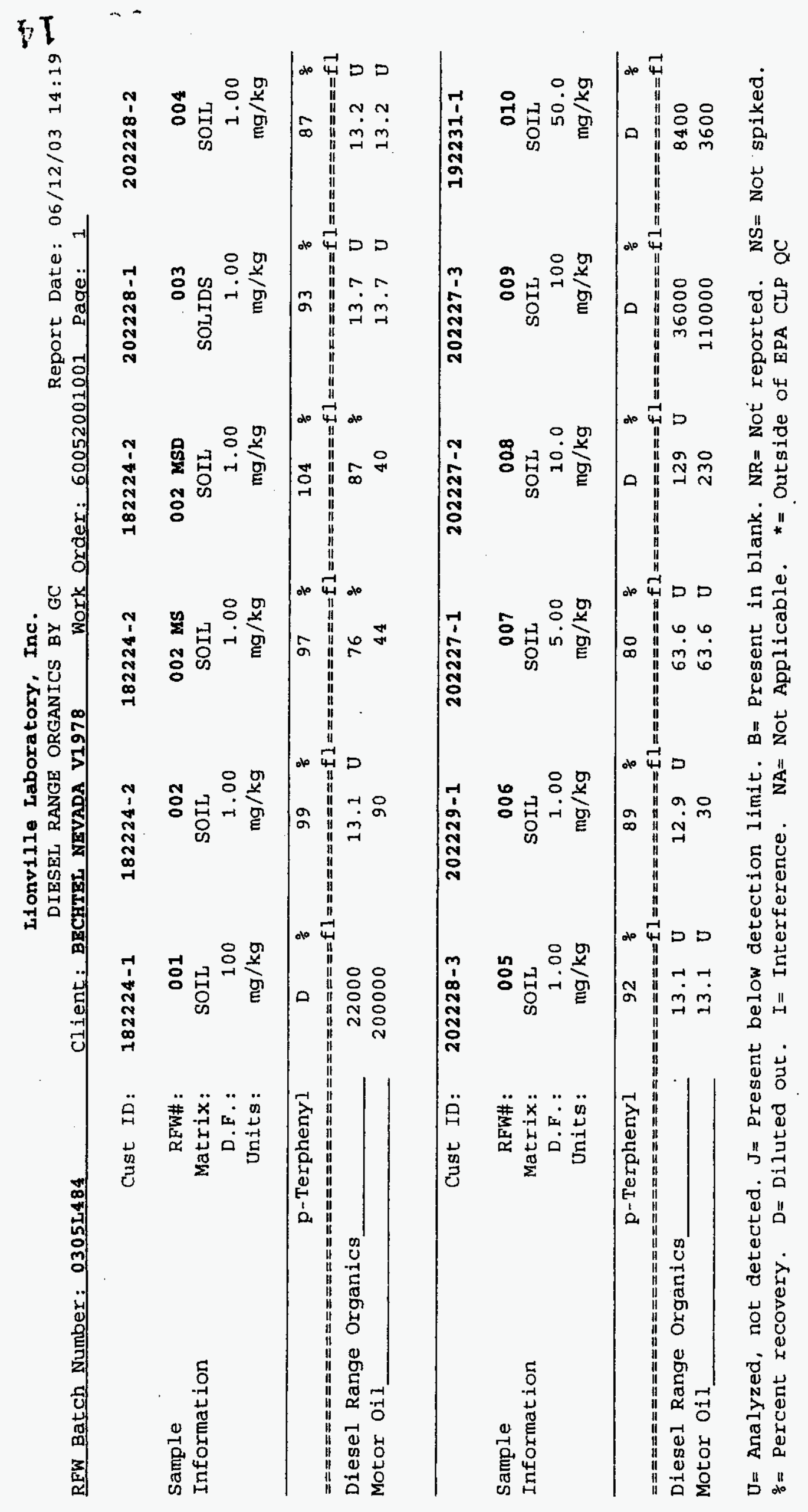


SI

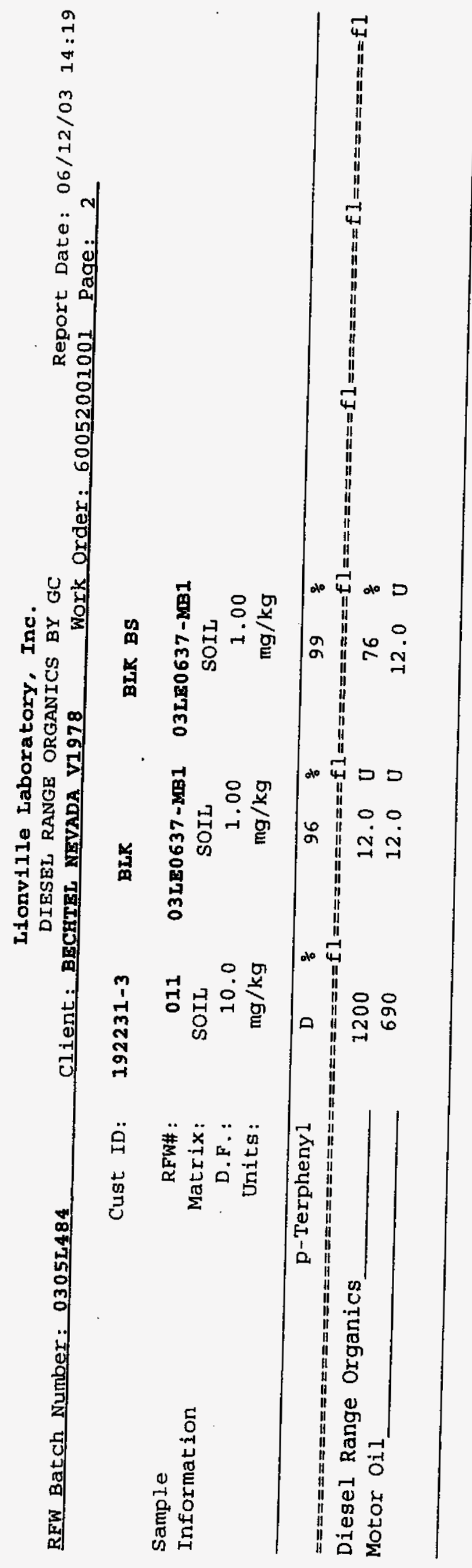

ช่ 목

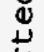

잉

4

苍告

哇范

范 *

용

독

可

要总

"1

资

E

$\leadsto$

S

范

(4)

ชั

몸

ค

芑苛

㟧

Q.

ค 苛

뭄

"

○

$\stackrel{0}{0}$

范

ㄴ

草莫<smiles>[C]1[C]C=C1</smiles>

है 

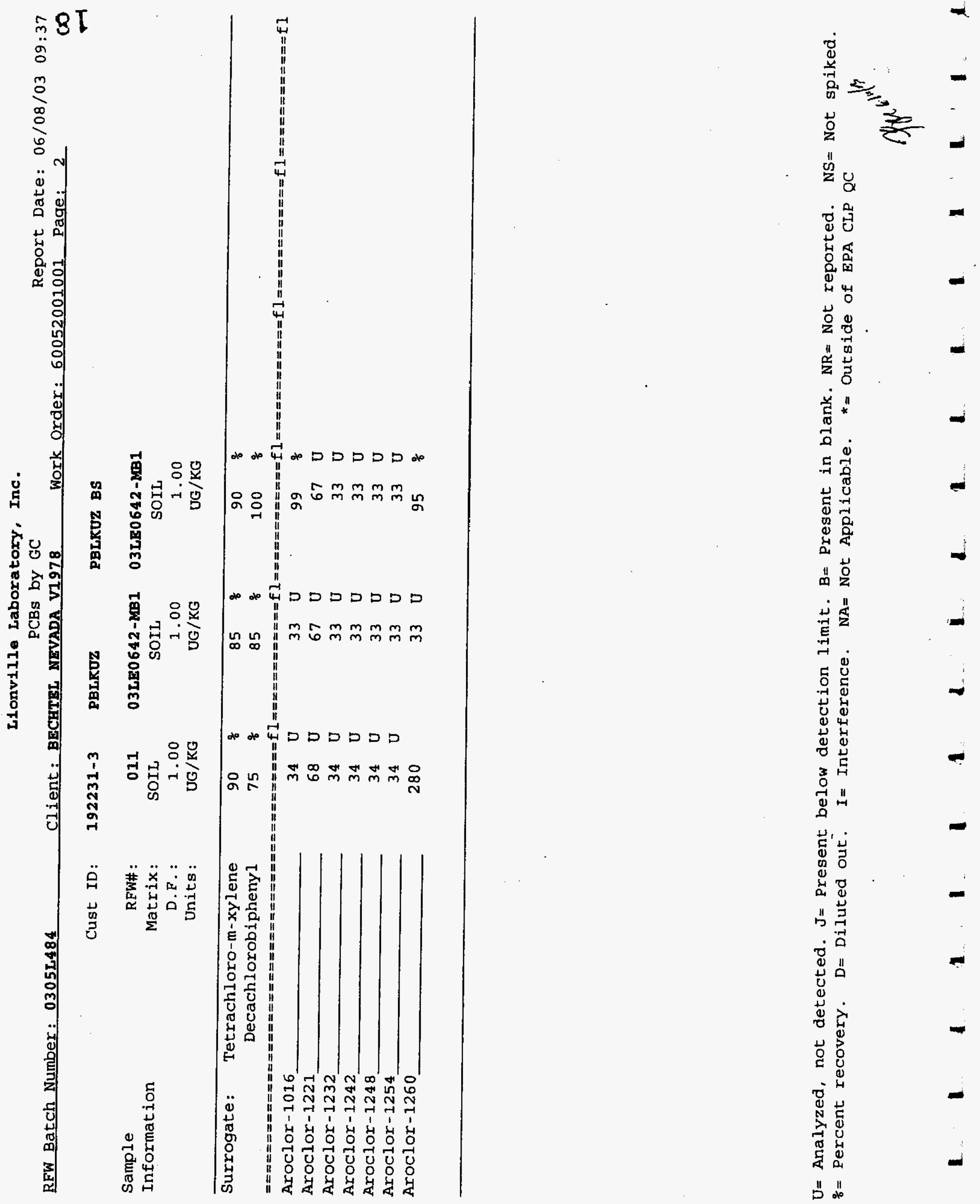


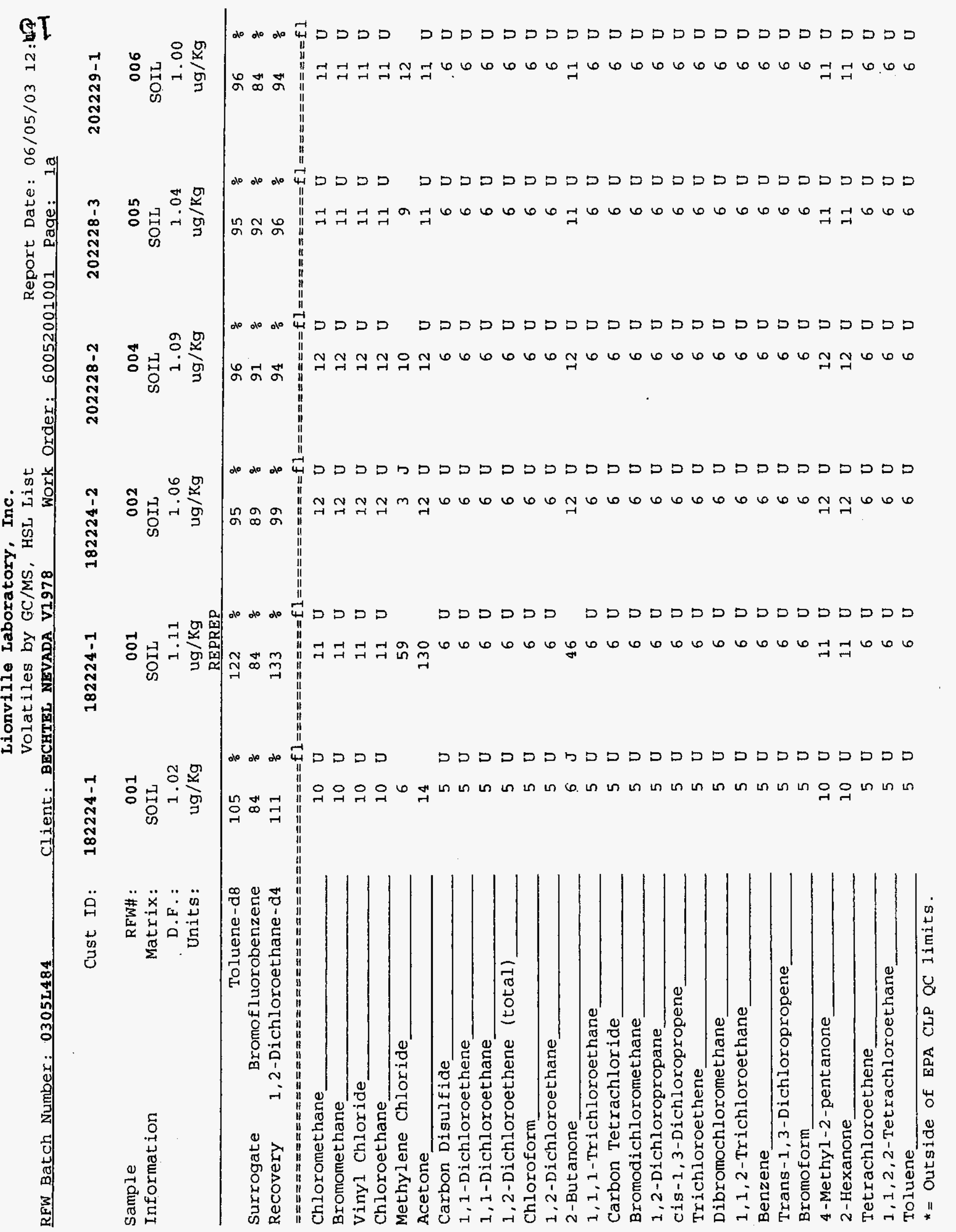




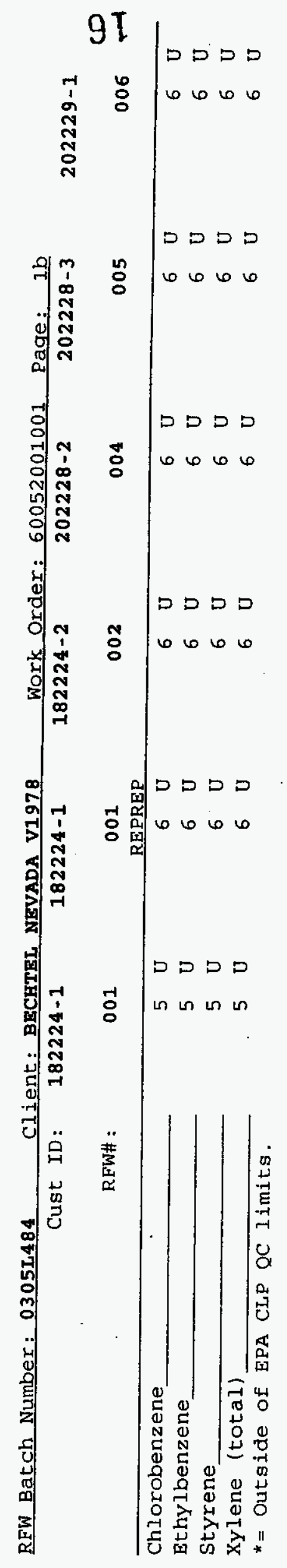




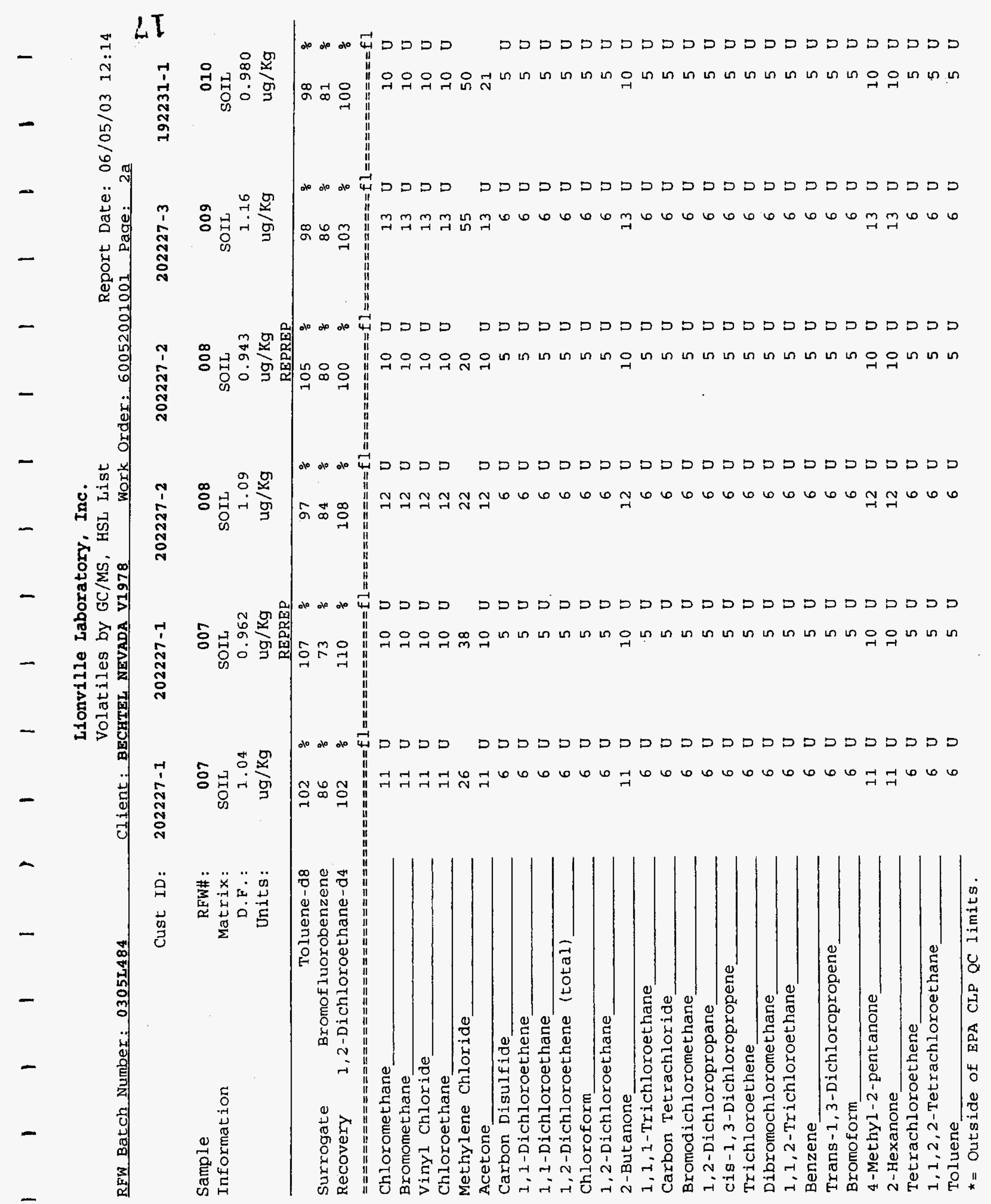




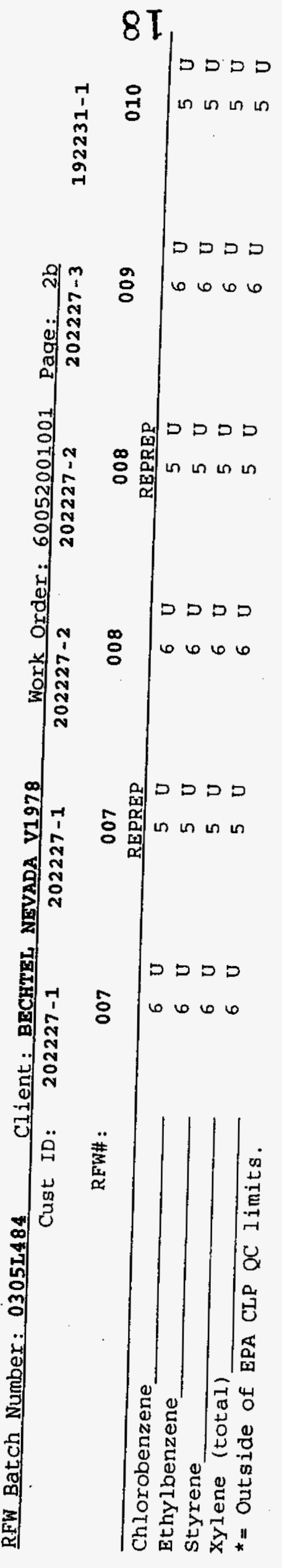




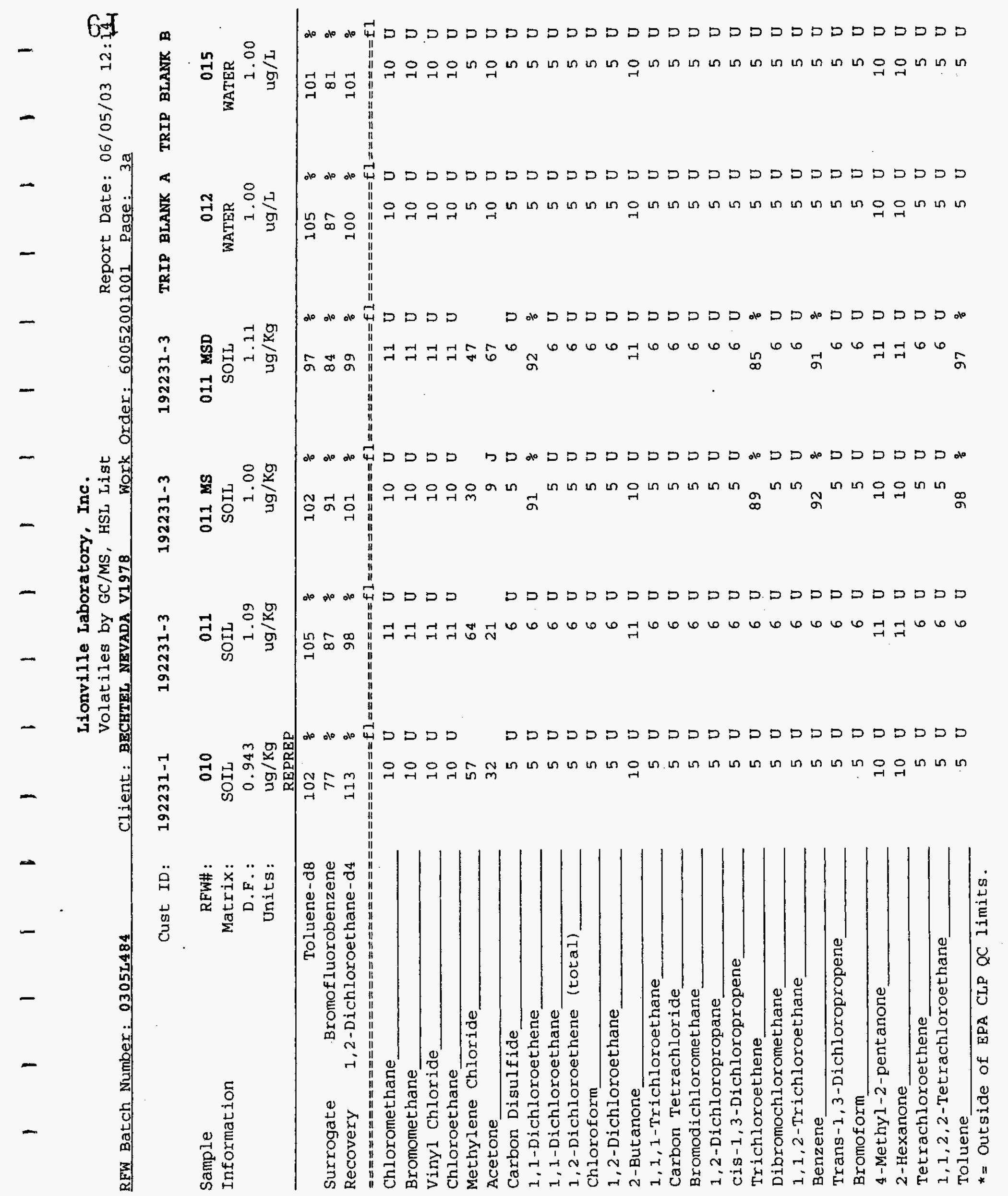




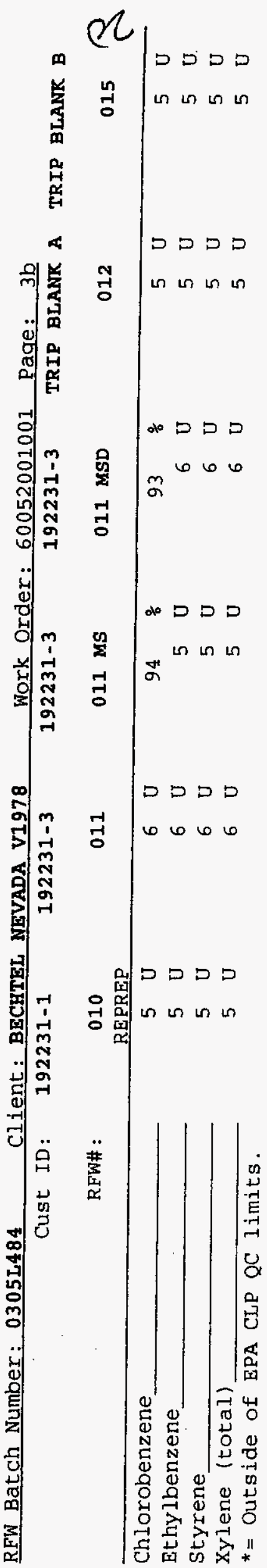




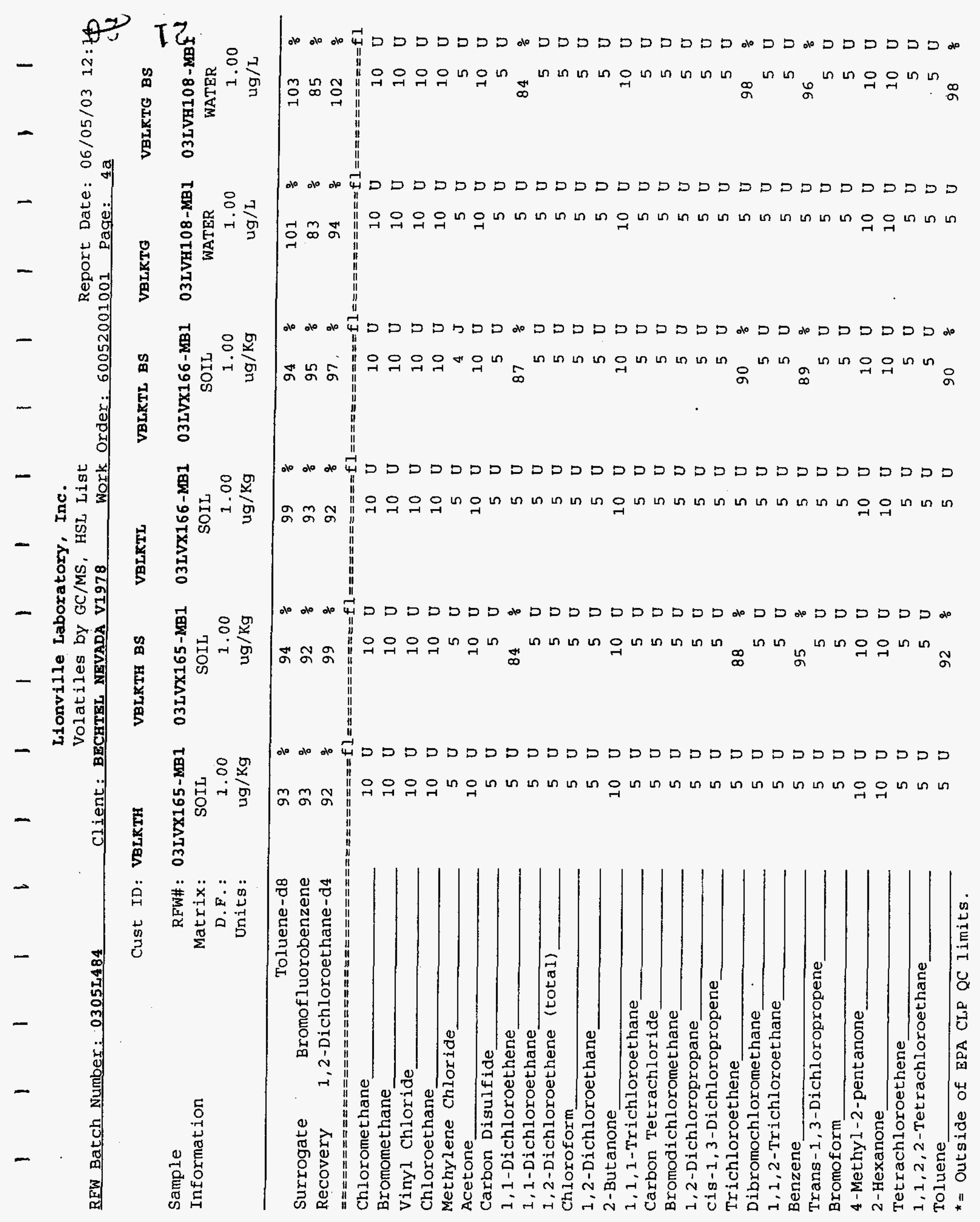




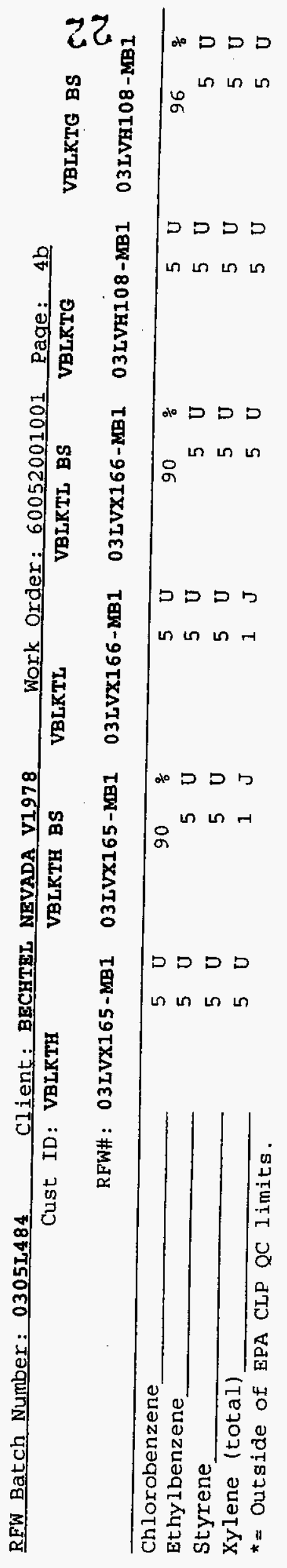


$-\quad B I$
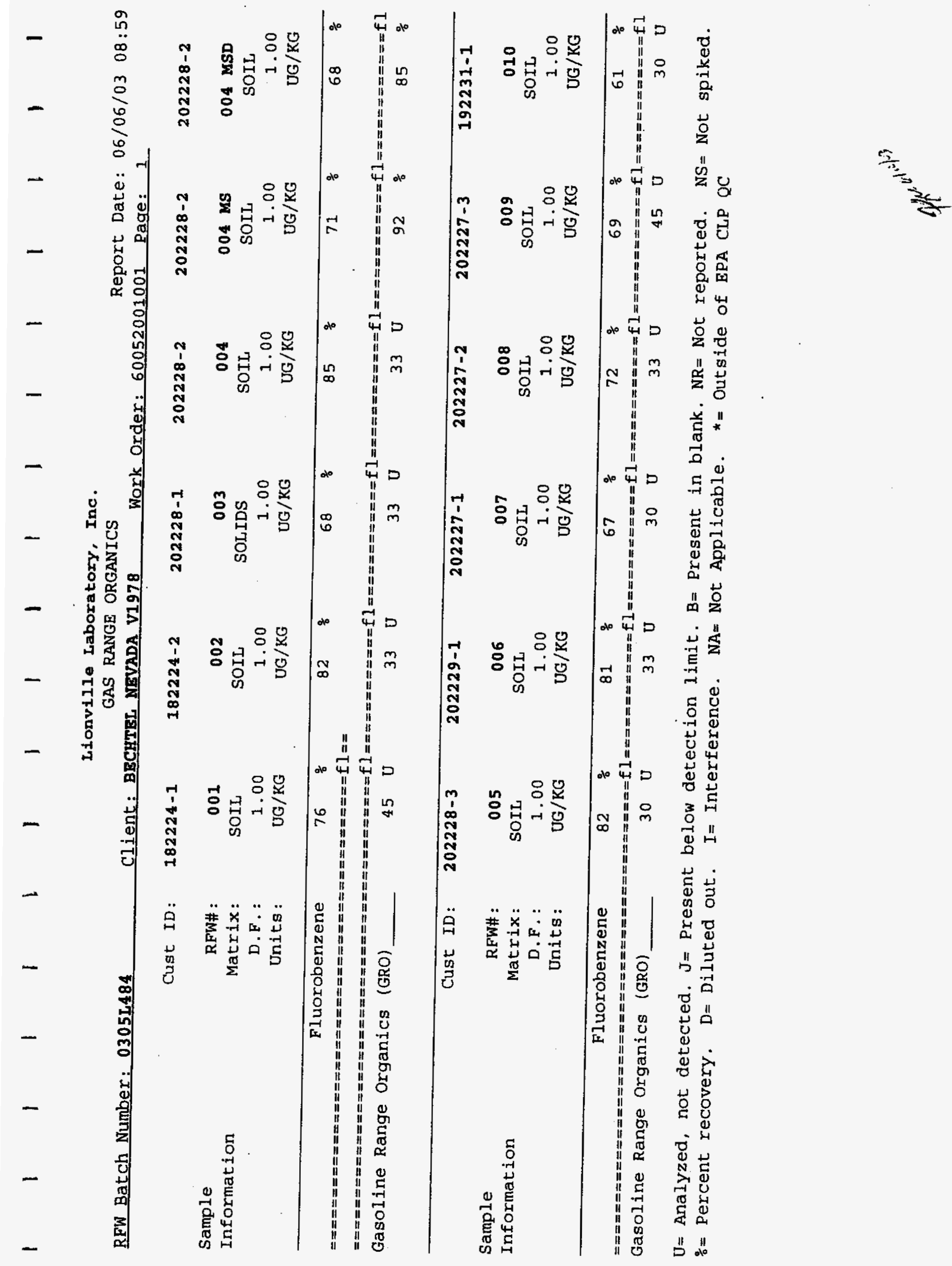
GI

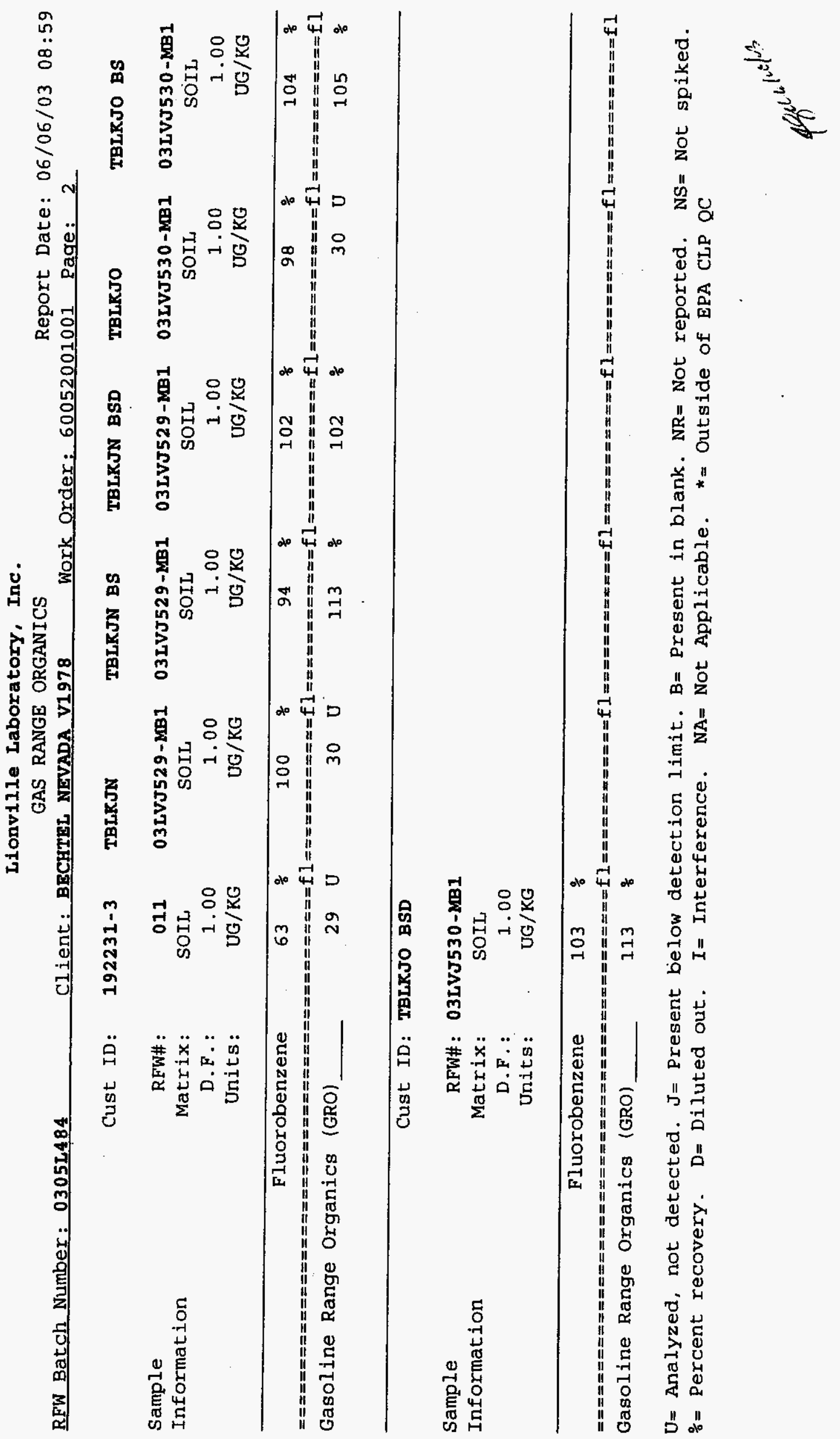




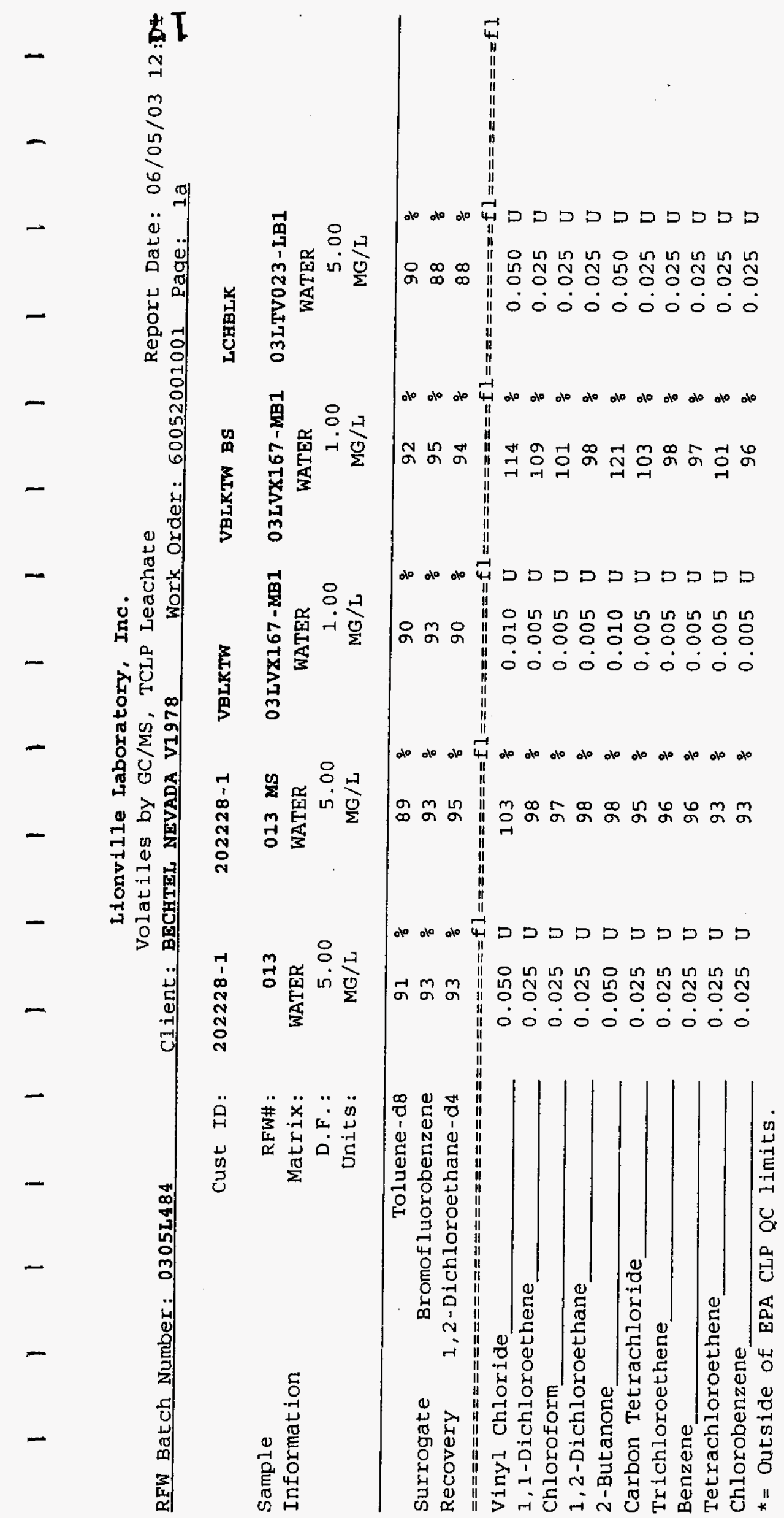




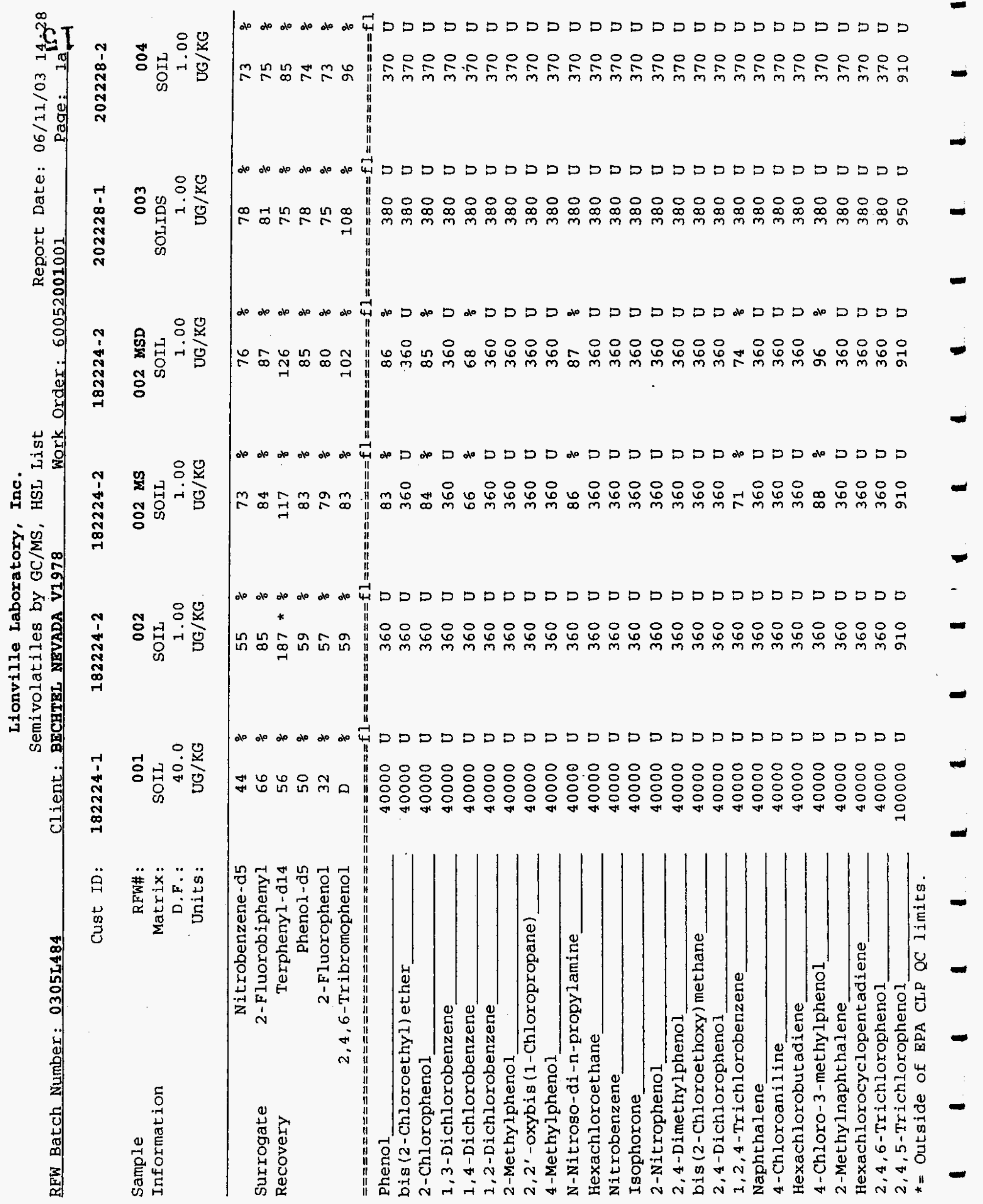




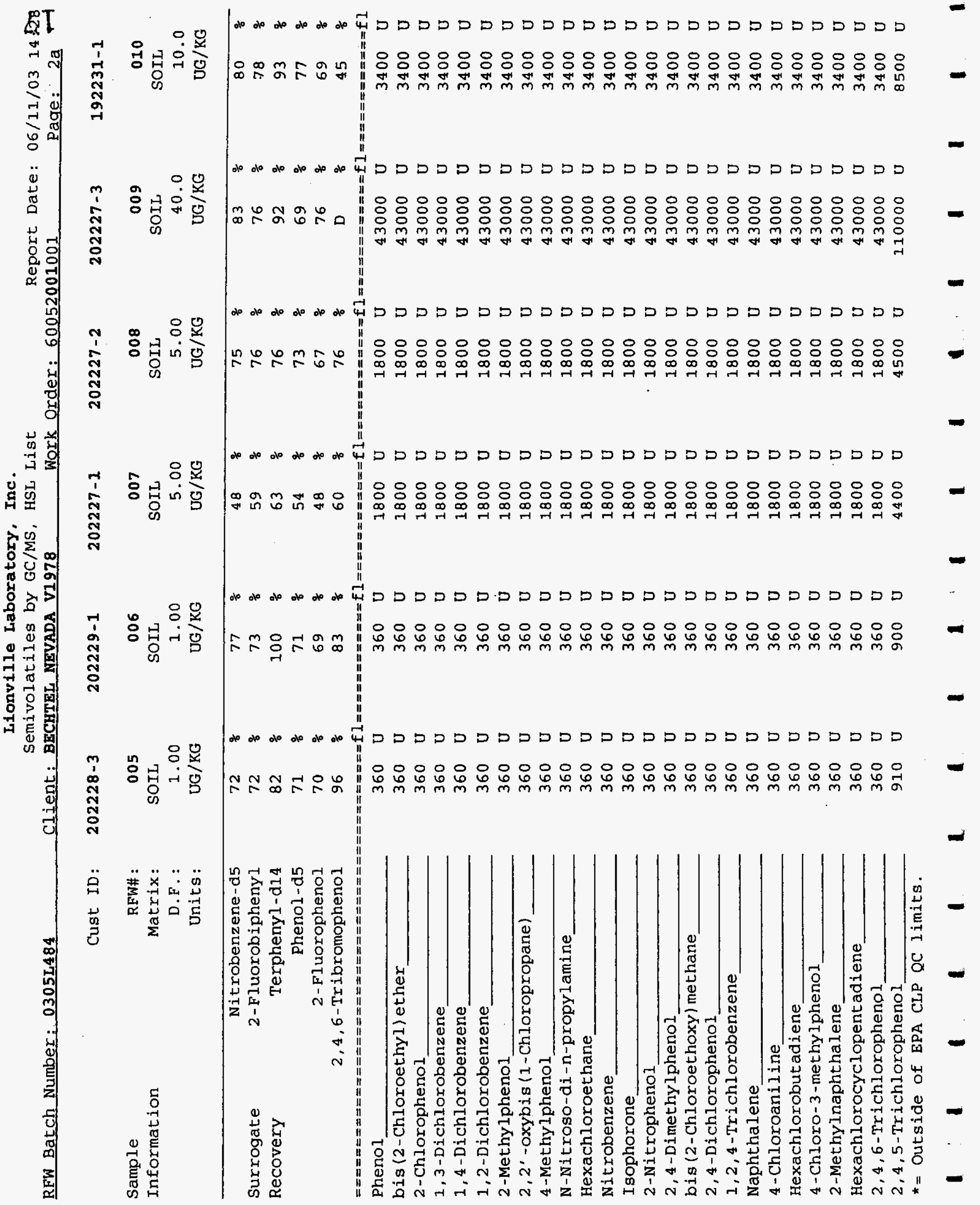




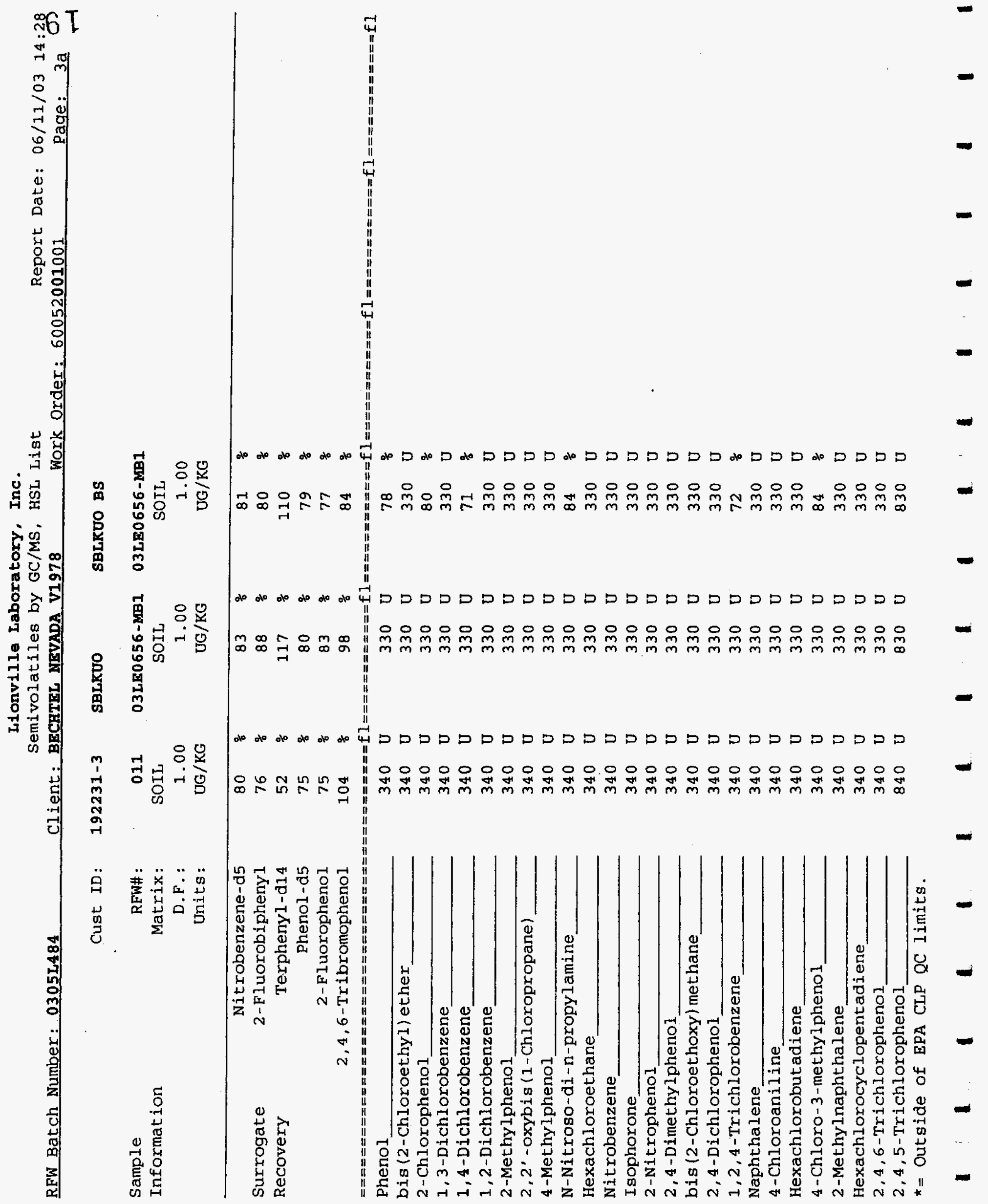




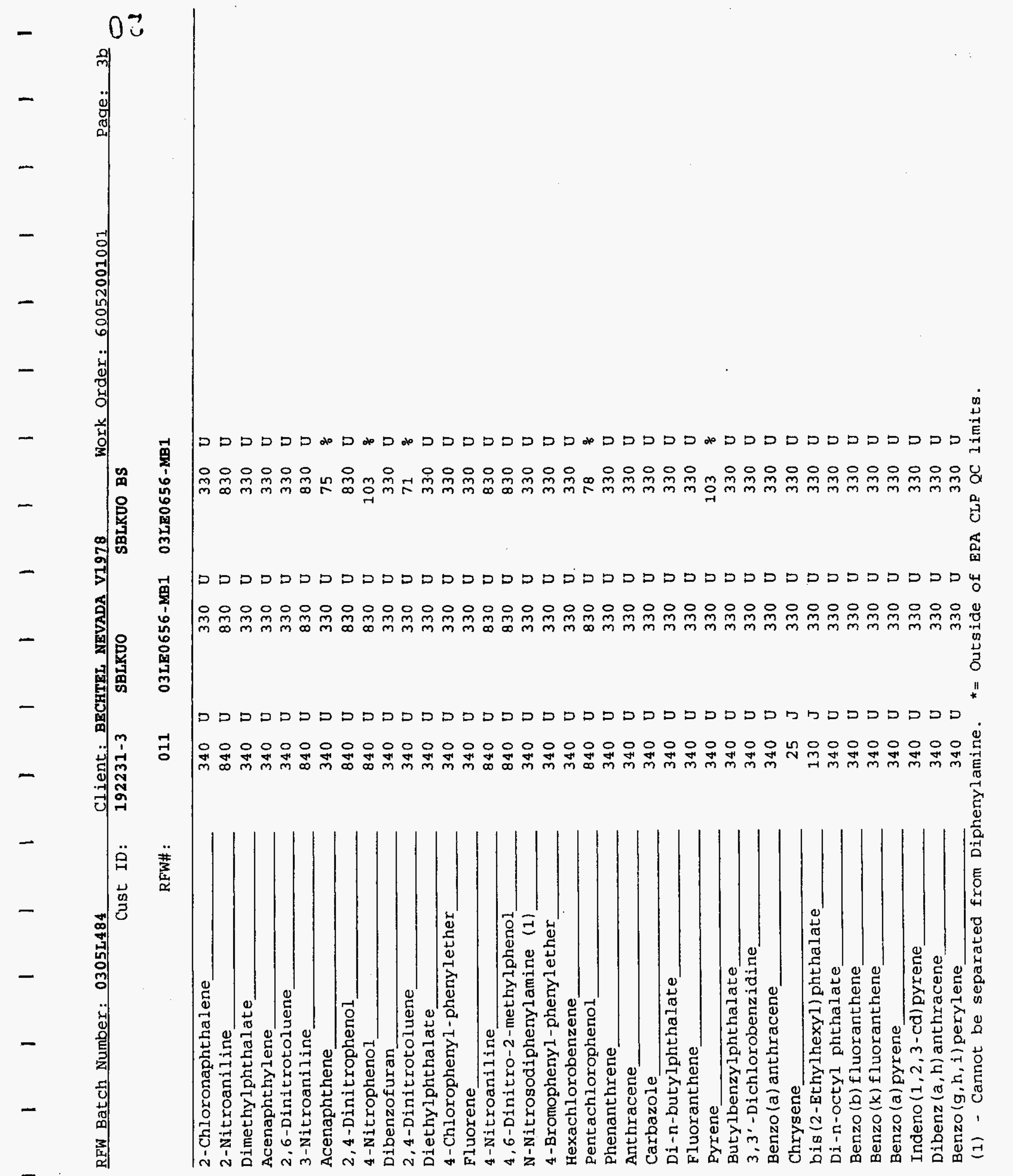


Closure Report - CAU 523

Section: Appendix A

Revision: 0

Date: November 2003
$-$

$-$

THIS PAGE INTENTIONALLY LEFT BLANK 
Closure Report - CAU 523

Section: Appendix A

Revision: 0

Date: November 2003

\section{SAMPLE DELIVERY GROUP}

V1979 
Date: November 2003 


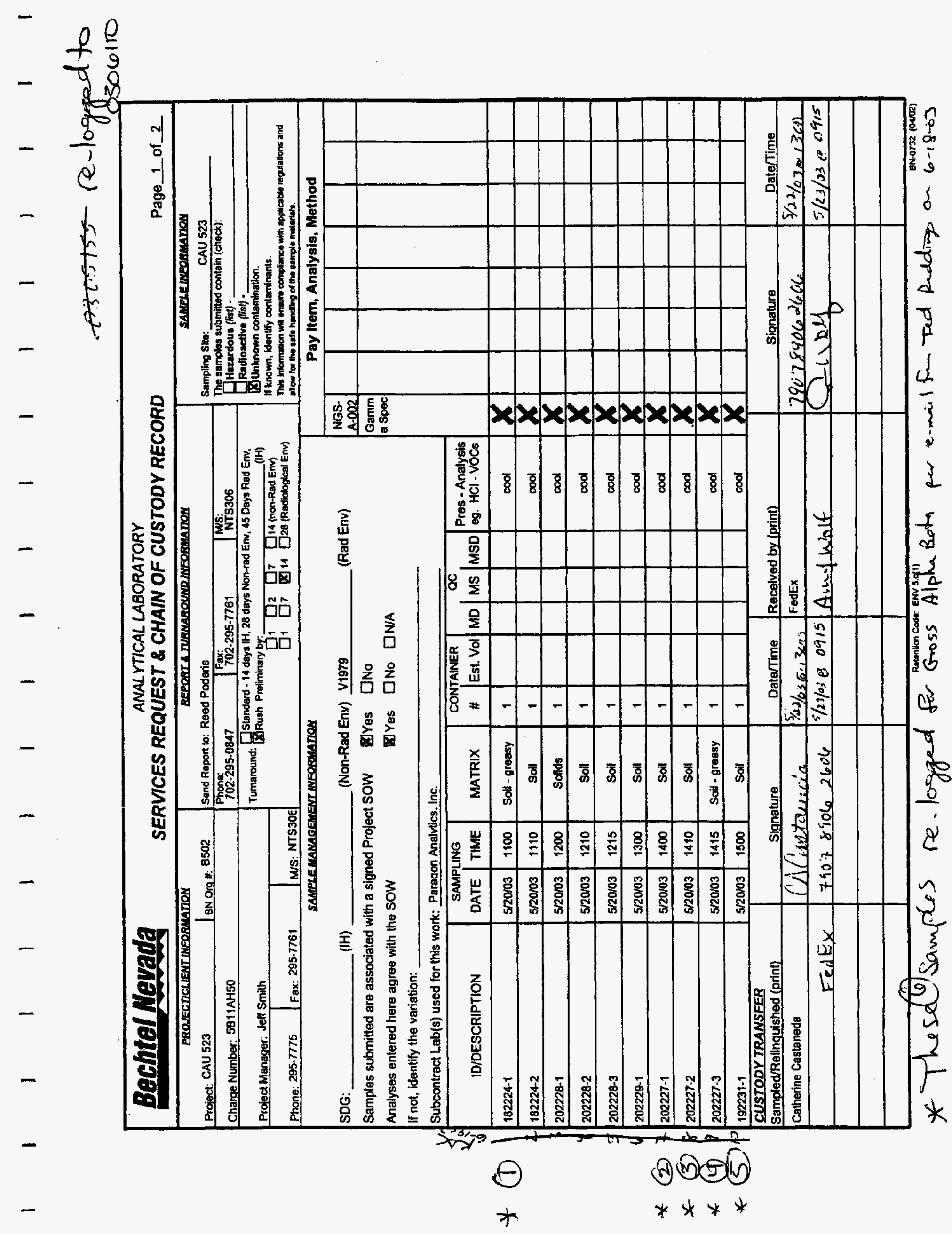



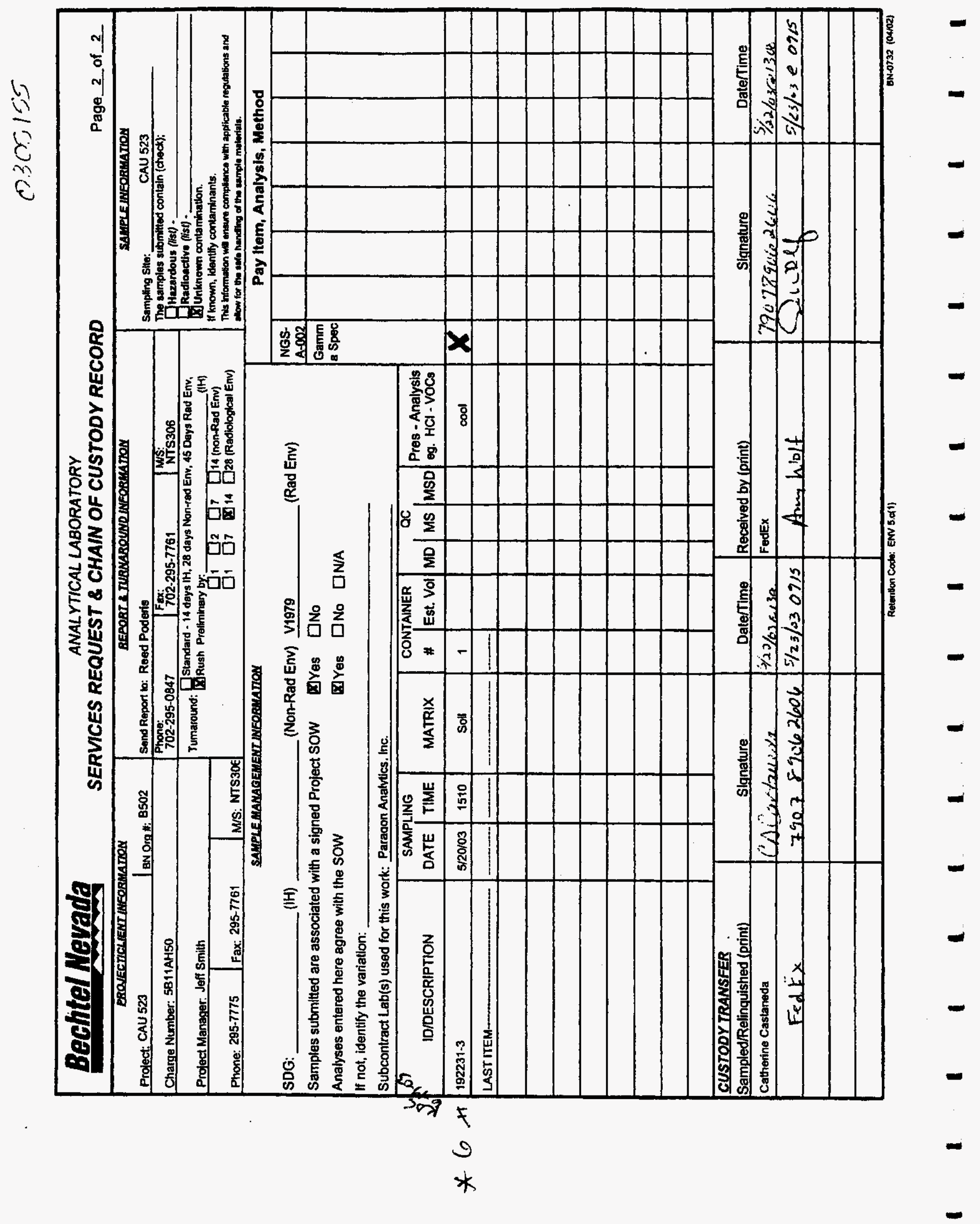


\section{Gamma Spectroscopy Results}

\section{Method PAl 713R8}

\section{Sample Results}

Client Name: Bechtel Nevada

Client Project Name: CAU 523

Client Project Number: V1979
Page: 1 of 22

Reported on: Thursday, May 29, 2003

10:41:44

Laboratory Name: Paragon Analytics, Inc.

PAl Work Order: 0305155
Field 10: 182224-1

Lab ID:0305155-1
Sample Matrix: Soil

Date Prepared: 28-May-03

Prep SOP: PAI 739R6

Prep Batch: GS02052
Date Collected: 20-May-03

Date Analyzed: 28-May-03

Analytical SOP: PAI 713R8

Spectrum Code: 030653D09A
Final Aliquot: $65.60 \mathrm{~g}$

Report Basis: Dry Woight Count Time (min.): 30

LIbrary: LNG_GAM-A-00

\begin{tabular}{|c|c|c|c|c|}
\hline Target Nuclide & Result +1- 2 s TPU & MDC & $\begin{array}{c}\text { Reporting } \\
\text { Units }\end{array}$ & Lab Qualifier \\
\hline Ac-228 & $0.90+/ \cdot 0.81$ & 1.2 & $\mathrm{pCi} / \mathrm{g}$ & $u$ \\
\hline Am-241 & $0.29+/-0.79$ & 1.4 & $\mathrm{pCl} / \mathrm{g}$ & u \\
\hline $\mathrm{Ce}-144$ & $-0.38+1-0.54$ & 1.1 & $\mathrm{pCi} / \mathrm{g}$ & $U$ \\
\hline Co-60 & $0.10+/-0.15$ & 0.24 & $\mathrm{pCl} / \mathrm{g}$ & $U$ \\
\hline Cs-134 & $-0.17+/-0.19$ & 0.38 & $\mathrm{pCi} / \mathrm{g}$ & $U$ \\
\hline Cs-137 & $0.32+1-0.21$ & 0.27 & $\mathrm{pCl} / \mathrm{g}$ & $T$ \\
\hline Eu-152 & $0.14+/-0.28$ & 0.38 & $\mathrm{pCi} / \mathrm{g}$ & $u$ \\
\hline Eu-154 & $0.26+/-0.92$ & 1.7 & $\mathrm{pCi} / \mathrm{g}$ & $\mathrm{u}$ \\
\hline Eu-155 & $-0.11+1-0.32$ & 0.61 & $\mathrm{pCl} / \mathrm{g}$ & $u$ \\
\hline$K-40$ & $7.7+/-4.2$ & 5.4 & $\mathrm{pCi} / \mathrm{g}$ & \\
\hline $\mathrm{Pb}-212$ & $0.55+/-0.28$ & 0.37 & $\mathrm{pCi} / \mathrm{g}$ & \\
\hline $\mathrm{Pm}-144$ & $-0.11+/-0.17$ & 0.35 & $\mathrm{pCi} / \mathrm{g}$ & $\bar{U}$ \\
\hline $\mathrm{Pm}-146$ & $0.09+1-0.15$ & 0.26 & $\mathrm{pCi} / \mathrm{g}$ & $u$ \\
\hline Ru-106 & $-0.4+1-1.1$ & 2.4 & $\mathrm{pCi} / \mathrm{g}$ & U \\
\hline $\mathrm{Sb}-125$ & $-0.06+1-0.33$ & 0.64 & $\mathrm{pCi} / \mathrm{g}$ & U \\
\hline Th-234 & $-1.7+1-1.8$ & 3.6 & $\mathrm{pCi} / \mathrm{g}$ & U \\
\hline U-235 & $-0.50+1-0.52$ & 1.1 & $\mathrm{pCl} / \mathrm{g}$ & U \\
\hline$Y-88$ & $0.07+/-0.19$ & 0.34 & $\mathrm{pCi} / \mathrm{g}$ & $\bar{U}$ \\
\hline
\end{tabular}

Data Package ID: GSS0305155-1 


\section{Gamma Spectroscopy Results}

\section{Method PAI 713R8}

\section{Sample Results}

Client Name: Bechtel Nevada

Client Project Name: CAU 523
Page: 2 of 22

Reported on: Thursday, May 29, 2003 10:41:44

Laboratory Name: Paragon Analytics, Inc. PAI Work Order: 0305155
Fiald ID: 182224-1

Lab ID:0305155-1
Sample Matrix: Soil

Date Prepared: 28-May-03

Prep SOP: PAI 739R6

Prep Batch: GS02052
Date Collected: 20-May-03

Date Analyzed: 28-May-03

Analytical SOP: PAI 713R8

Spectrum Code: 030653009A
Final Aliquot: $65.60 \mathrm{~g}$

Report Basis: Dry Weight

Count Time (min.): 30

Library: LNG_GAM-A-00

\begin{tabular}{|c|c|c|c|c|}
\hline Target Nuclide & Result +/- 2 s TPU & MDC & $\begin{array}{l}\text { Reporting } \\
\text { Units }\end{array}$ & Lab Quallfier \\
\hline
\end{tabular}

\section{Comments:}

Qualfions/Fisog:

$U$-Result is less than the sample specific MDC or less than the associated TPU.

Y1 - Chamical Yetd is in control at 100-110\%. Ouantitative Yield is assumed.

Y2 - Chemical Yield outside default imits.

LT - Result is less than Requested MDC, greater than sample specific MDC.

SQ - Spectral quality prevents accurate quentitation.

S1 - Nuclide idenlification and/or quantitation is tentative.

Tl - Nuclide identification is tentative.

$R$ - Nuclide has exceeded a halifives.
Abbreviations:

TPU - Total Propagated Uncertainty (see PAI SOP 743)

MDC - Minimum Delectable Concentration (see PAI SOP 709) 


\section{Gamma Spectroscopy Results}

\section{Method PAI 713R8}

\section{Sample Results}

Client Name: Bechtel Nevada

Client Project Name: CAU 523

Client Project Number: V1979
Page: 3 of 22

Reported on: Thursday, May 29, 2003 10:41:47

Laboratory Name: Paragon Anaiytics, Inc.

PAl Work Order: 0305155
Fieid ID: 182224-2

Lab ID:0305155-2
Sample Matrix: Soil

Date Prepared: 28-May-03

Prep SOP: PAI 739R6

Prep Batch: GS02051
Date Collected: 20-May-03

Date Analyzed: 28-May-03

Analytical SOP: PAI 713R8

Spectrum Code: 030630D06A
Final Aliquot: $381.2 \mathrm{~g}$

Report Basis: Dry Weight

Count Time (min.): 30

Library: LNG_GAM-A-00

\begin{tabular}{|c|c|c|c|c|}
\hline Target Nuclide & Result +1- 2 s TPU & MDC & $\begin{array}{l}\text { Reporting } \\
\text { Units }\end{array}$ & Lab Qualifier \\
\hline$A c-228$ & $2.58+1-0.59$ & 0.73 & $\mathrm{pCi} / \mathrm{g}$ & \\
\hline Am-241 & $0.42+/-0.46$ & 0.74 & $\mathrm{pCl} / \mathrm{g}$ & $\mathrm{U}$ \\
\hline$B \mathrm{i}-212$ & $3.0+/-1.8$ & 2.5 & $\mathrm{pCi} / \mathrm{g}$ & \\
\hline $\mathrm{Bi}-214$ & $1.38+/-0.39$ & 0.39 & $\mathrm{pCi} / \mathrm{g}$ & \\
\hline $\operatorname{Cd}-109$ & $5.9+/-2.5$ & 3.4 & $\mathrm{pCi} / \mathrm{g}$ & SI \\
\hline $\mathrm{C}_{\theta-144}$ & $-0.33+1-0.47$ & 0.83 & $\mathrm{pCi} / \mathrm{g}$ & $U$ \\
\hline Co-60 & $-0.07+1-0.11$ & 0.22 & $\mathrm{pCi} / \mathrm{g}$ & $U$ \\
\hline Cs-134 & $0.01+/-0.12$ & 0.21 & $\mathrm{pCl} / \mathrm{g}$ & $u$ \\
\hline Cs-137 & $0.126+/-0.094$ & 0.14 & $\mathrm{pCi} / \mathrm{g}$ & $\bar{U}$ \\
\hline Eu-152 & $0.26+/-0.48$ & 0.83 & $\mathrm{pCi} / \mathrm{g}$ & $U$ \\
\hline Eu-154 & $0.01+/-0.57$ & 1.0 & $\mathrm{pCl} / \mathrm{g}$ & $u$ \\
\hline Eu-155 & $0.18+1-0.18$ & 0.29 & $\mathrm{pCi} / \mathrm{g}$ & $\mathrm{u}$ \\
\hline$k-40$ & $27.9+1-5.6$ & 2.6 & $\mathrm{pCi} / \mathrm{g}$ & \\
\hline $\mathrm{Pb}-212$ & $3.00+1-0.57$ & 0.27 & $\mathrm{pCi} / \mathrm{g}$ & \\
\hline $\mathrm{Pb}-214$ & $1.49+1-0.34$ & 0.32 & $\mathrm{pCi} / \mathrm{g}$ & \\
\hline $\mathrm{Pm}-144$ & $-0.029+1-0.090$ & 0.17 & $\mathrm{pCi} / \mathrm{g}$ & $u$ \\
\hline $\mathrm{Pm}-146$ & $0.04+1-0.10$ & 0.18 & $\mathrm{pCi} / \mathrm{g}$ & U \\
\hline Ru-106 & $-0.42+1-0.77$ & 1.5 & $\mathrm{pCi} / \mathrm{g}$ & u \\
\hline Sb-125 & $0.09+1-0.22$ & 0.40 & $\mathrm{pCi} / \mathrm{g}$ & $v$ \\
\hline Th-234 & $4.4+/-2.2$ & 3.3 & $\mathrm{pCi} / \mathrm{g}$ & $T$ \\
\hline$T t-208$ & $0.78+/-0.20$ & 0.18 & $\mathrm{pCi} / \mathrm{g}$ & \\
\hline U-235 & $-0.08+1-0.45$ & 0.78 & $\mathrm{pCi} / \mathrm{g}$ & $\mathrm{u}$ \\
\hline $\mathrm{Y}-88$ & $-0.053+1-0.096$ & 0.18 & $\mathrm{pCl} / \mathrm{g}$ & $u$ \\
\hline
\end{tabular}

Data Package ID: GSS0305155-1

Paragon Analytics Inc. 


\section{Gamma Spectroscopy Results}

\section{Method PAI 713R8}

\section{Sample Results}

Client Name: Bechtel Nevada

Client Project Name: CAU 523 Client Project Number: V1979
Page: 4 of 22

Reported on: Thursday, May 29, 2003

10:41:47

Laboratory Name: Paragon Analytics, Inc.

PAl Work Order: 0305155
Field ID: 182224-2

Lab ID:0305155-2
Sample Matrix: Soil

Date Prepared: 28-May-03

Prep SOP: PAI 739R6

Prep Batch: GS02051
Date Collected: 20-May-03

Date Analyzed: 28-May-03

Analytical SOP: PAI 713R8

Spectrum Code: 030630D06A
Final Aliquot: $381.2 \mathrm{~g}$

Report Basis: Dry Weight Count Time (min.): 30

Library: LNG_GAM-A-00

\begin{tabular}{|c|c|c|c|c|}
\hline Target Nuclide & Result +1.2 s TPU & MDC & $\begin{array}{c}\text { Reporting } \\
\text { Units }\end{array}$ & Lab Qualifier \\
\hline
\end{tabular}

\section{Comments:}

\section{Qualiflars/Flags:}

$U$ - Result is less than the sample specific MOC or less than the associated TPU.

Y1 - Chemical Yiald is in control at 100-110\%. Quantitative Yield is assumed.

Y2 - Chemical Yield outside default limihs.

LT - Result is less than Requested MDC, grealer than sample specific MDC.

SQ - Spectral quality prevents accurate quantitation.

SI - Nuclide identification and/or quantitation is tentative.

TI - Nuclide identification is tentative.

$R$ - Nuclide has exceeded 8 halfives.
Abbreviations:

TPU - Totad Propagated Uncertainty (see PAI SOP 743)

MDC - Minimum Detectable Concentration (see PAI SOP 709)

Data Package ID: GSS0305155-1 


\title{
Gamma Spectroscopy Results
}

\section{Method PAI 713R8}

\author{
Sample Duplicate Results
}

Client Name: Bechtel Nevada

Client Project Name: CAU 523

Client Project Number: V1979

Field ID: $182224-2$

Lab ID:0305155-2-D1
Sample Matrix: Soil

Date Prepared: 28-May-03

Prep SOP: PAI 739R6

Prep Batch: GS02051

Page: 1 of 6

Reported on: Thursday, May 29, 2003

$10: 41: 47$

Laboratory Name: Paragon Analytics, Inc.

PAI Work Order: 0305155

\begin{tabular}{|c|c|c|c|c|}
\hline Target Nuclide & Result $+/-2$ s TPU & MDC & $\begin{array}{c}\text { Reporting } \\
\text { Units }\end{array}$ & Lab Qualifier \\
\hline Ac-228 & $2.68+/ \cdot 0.59$ & 0.64 & $\mathrm{pCi} / \mathrm{g}$ & \\
\hline Am-241 & $-0.23+/-0.64$ & 1.1 & $\mathrm{pCi} / \mathrm{g}$ & U \\
\hline $\mathrm{Bi}-212$ & $2.5+/-1.3$ & 1.6 & $\mathrm{pCi} / \mathrm{g}$ & \\
\hline $\mathrm{Bi}-214$ & $1.40+/-0.36$ & 0.31 & $\mathrm{pCi} / \mathrm{g}$ & \\
\hline $\mathrm{Ce}-144$ & $0.12+1-0.48$ & 0.82 & $\mathrm{pCi} / \mathrm{g}$ & $\mathrm{U}$ \\
\hline $\mathrm{Co}-60$ & $-0.011+/-0.094$ & 0.18 & $\mathrm{pCi} / \mathrm{g}$ & U \\
\hline Cs-134 & $0.018+1-0.082$ & 0.14 & $\mathrm{pCi} / \mathrm{g}$ & U \\
\hline Cs-137 & $0.25+/-0.41$ & 0.13 & $\mathrm{pCl} / \mathrm{g}$ & \\
\hline Eu-152 & $-0.08+1-0.42$ & 0.82 & $p \mathrm{Ci} / \mathrm{g}$ & $\mathrm{U}$ \\
\hline Eu-154 & $-0.21+/-0.41$ & 0.83 & $\mathrm{pCi} / \mathrm{g}$ & U \\
\hline Eu-155 & $0.09+/-0.28$ & 0.47 & $\mathrm{pCi} / \mathrm{g}$ & $U$ \\
\hline$K-40$ & $30.2+1-5.9$ & 1.4 & $\mathrm{pCi} / \mathrm{g}$ & \\
\hline $\mathrm{Pb}-212$ & $2.72+/-0.53$ & 0.27 & $\mathrm{pCi} / \mathrm{g}$ & \\
\hline $\mathrm{Pb}-214$ & $1.83+/=0.38$ & 0.27 & $\mathrm{pCi} / \mathrm{g}$ & \\
\hline$P m-144$ & $-0.014+/-0.079$ & 0.15 & $\mathrm{pCi} / \mathrm{g}$ & U \\
\hline$P m-146$ & $0.01+\% 0.10$ & 0.18 & $\mathrm{pCi} / \mathrm{g}$ & $U$ \\
\hline Ru-106 & $0.26+1-0.82$ & 1.4 & $\mathrm{pCi} / \mathrm{g}$ & $U$ \\
\hline Sb-125 & $0.03+/-0.19$ & 0.35 & $\mathrm{pCi} / \mathrm{g}$ & $U$ \\
\hline Th-234 & $2.5+/-1.9$ & 2.9 & $\mathrm{pCi} / \mathrm{g}$ & U \\
\hline TI-208 & $1.04+1-0.23$ & 0.15 & $\mathrm{pCi} / \mathrm{g}$ & \\
\hline $\mathrm{U}-235$ & $0.25+/-0.51$ & 0.85 & $\mathrm{pCi} / \mathrm{g}$ & U \\
\hline$Y-88$ & $0.037+/-0.096$ & 0.17 & $\mathrm{pCl} / \mathrm{g}$ & U \\
\hline
\end{tabular}

Data Package ID: GSS0305155-1 


\section{Gamma Spectroscopy Results}

\section{Method PAI 713R8}

Sample Duplicate Results

\begin{abstract}
Client Name: Bechtel Nevada
Client Project Name: CAU 523

Client Project Number: V1979
\end{abstract}

Page: 2 of 6

Reported on: Thursday, May 29, 2003 10:41:47

Laboratory Name: Paragon Analytics, Inc. PAI Work Order: 0305155

Fieid ID: $182224-2$

Lab ID:0305155-2-D1
Sample Matrix: Soil

Date Prepared: 28-May-03

Prep SOP: PAI $739 R 6$

Prep Batch: GS02051
Date Collected: 20-May-03

Date Analyzed: 28-May-03

Analytical SOP: PAI 713R8

Spectrum Code: 030513003A
Final Aliquot: 381.2

Aliquot Units: $\mathrm{g}$

Report Basis: Dry Weight Count Time (min.): 30

\begin{tabular}{|c|c|c|c|c|}
\hline Target Nuclide & Result $+/-2$ s TPU & MDC & $\begin{array}{c}\text { Reporting } \\
\text { Units }\end{array}$ & Lab Qualifier \\
\hline
\end{tabular}

\section{Comments:}

\section{Quatitions/Flags:}

$U$ - Result is less than the sample spectic MDC or less than the associated TPU.

Y1 - Chemical Yield is in control at 100-110\%. Quantitative yield is assumed.

Y2 - Chemical Yleid outside default limits.

- Duplicate DER not within control limits.

LT - Result is less than Requested MDC. greater than sample specific MDC.

SQ-Spectral quelity prevents eccurate quantitation.

SI - Nuclide identification and/or quantitation is lentative.

TI - Nuclide identification is tentative.

$R$ - Nuclide has exceeded 8 halfives.
Abbreviations:

TPU - Toial Propagated Uncenainty (see PAI SOP 743)

MDC - Minimum Delectable Concéntration (see PAI SOP 709)

Data Package ID: GSS0305155-1 


\section{Gamma Spectroscopy Results}

\section{Method PAI 713R8}

\section{Sample Results}

Client Name: Bechtel Nevada

Client Project Name: CAU 523

Client Project Number: V1979
Page: 5 of 22

Reported on: Thursday, May 29, 2003

10:41:49

Laboratory Name: Paragon Analytics, Inc.

PAI Work Order: 0305155
Field ID:202228-1

Lab ID:0305155-3
Sample Matrix: Solid

Date Prepared: 28-May-03

Prep SOP: PAl 739R6

Prep Batch: GS02053
Date Collected: 20-May-03

Date Analyzed: 28-May-03

Analytical SOP: PAI $713 R 8$

Spectrum Code: 030798D01A
Final Aliquot: $258.0 \mathrm{~g}$

Report Basis: Dry Weight Count Time (min.): 30

Library: LNG_GAM-A-00

\begin{tabular}{|c|c|c|c|c|}
\hline Target Nuclide & Result $+/-2$ s TPU & MDC & $\begin{array}{c}\text { Reporting } \\
\text { Units }\end{array}$ & Lab Qualifier \\
\hline Ac-228 & $0.22+1-0.22$ & 0.35 & $\mathrm{pCi} / \mathrm{g}$ & U \\
\hline$A m-241$ & $0.19+1-0.23$ & 0.38 & $\mathrm{pCl} / \mathrm{g}$ & $u$ \\
\hline Ce-144 & $0.01+/-0.26$ & 0.46 & $\mathrm{pCi} / 9$ & $U$ \\
\hline $\mathrm{Co}-60$ & $-0.039+1-0.068$ & 0.13 & $\mathrm{pCi} / \mathrm{g}$ & U \\
\hline Cs-134 & $0.003+/=0.091$ & 0.16 & $\mathrm{pCi} / \mathrm{g}$ & U \\
\hline Cs-137 & $0.025+/-0.057$ & 0.097 & $\mathrm{pCi} / \mathrm{g}$ & $U$ \\
\hline Eu-152 & $0.08+1-0.30$ & 0.53 & $\mathrm{pCi} / \mathrm{g}$ & $\mathrm{u}$ \\
\hline Eu-154 & $-0.17+1-0.28$ & 0.55 & $\mathrm{pCi} / \mathrm{g}$ & $U$ \\
\hline Eu-155 & $0.11+/-0.16$ & 0.27 & $\mathrm{pCi} / \mathrm{g}$ & $u$ \\
\hline$K-40$ & $5.9+1-1.6$ & 1.5 & $\mathrm{pCi} / \mathrm{g}$ & \\
\hline $\mathrm{Nb}-94$ & $0.063+\% \quad 0.066$ & 0.10 & $\mathrm{pCi} / \mathrm{g}$ & $u$ \\
\hline $\mathrm{Pb}-212$ & $0.33+/-0.13$ & 0.16 & $\mathrm{pCi} / \mathrm{g}$ & \\
\hline $\mathrm{Pb}-214$ & $0.32+/ .0 .14$ & 0.22 & $\mathrm{pCi} / \mathrm{g}$ & \\
\hline $\mathrm{Pm}-144$ & $-0.004+/-0.061$ & 0.11 & $\mathrm{pCi} / \mathrm{g}$ & $U$ \\
\hline $\mathrm{Pm}-146$ & $-0.019+1-0.067$ & 0.12 & $\mathrm{pCi} / \mathrm{g}$ & U \\
\hline Ru-106 & $0.03+1-0.54$ & 0.96 & $\mathrm{pCi} / \mathrm{g}$ & $\mathrm{u}$ \\
\hline Sb-125 & $-0.12+/-0.14$ & 0.27 & $\mathrm{pCi} / \mathrm{g}$ & 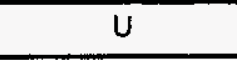 \\
\hline Th-234 & $0.77+/ .0 .92$ & 1.5 & $\mathrm{pCi} / \mathrm{g}$ & $u$ \\
\hline$U-235$ & $0.00+/-0.27$ & 0.48 & $\mathrm{pCi} / \mathrm{g}$ & $u$ \\
\hline$\gamma-88$ & $-0.034+1-0.062$ & 0.12 & $\mathrm{pCi} / \mathrm{g}$ & $u$ \\
\hline
\end{tabular}

Data Package ID: GSS0305155-1 


\section{Gamma Spectroscopy Results}

\section{Method PAl 713R8}

\section{Sample Results}

Client Name: Bechtel Nevada

Client Project Name: CAU 523 Client Project Number: V1979
Page: 6 of 22

Reported on: Thursday, May 29, 2003

10:41:49

Laboratory Name: Paragon Analytics, tnc.

PAI Work Order: 0305155
Field ID: 202228-1

Lab ID:0305155-3
Sample Matrix: Solid

Date Prepared: 28-May-03

Prep sOP: PAI 739R6

Prep Batch: . GS02053
Date Collected: 20-May-03

Date Analyzed: 28-May-03

Analytical SOP: PAI 713R8

Spectrum Code: 030798D01A
Final Aliquot: $258.0 \mathrm{~g}$

Report Basis: Dry Weight Count Time (min.): 30

Library: LNG_GAM-A-00

\begin{tabular}{|c|c|c|c|c|}
\hline Target Nuclide & Result +/- 2 s TPU & MDC & $\begin{array}{c}\text { Reporting } \\
\text { Units }\end{array}$ & Lab Qualifier \\
\hline
\end{tabular}

\section{Comments:}

\section{Oualifiera/Flags:}

$U$ - Result is less than the sample specific MDC or less than the associated TPU.

Y1 - Chemical Yleld is in control at 100-110\%. Quantitative Yletd is assumed.

Y2 - Chemical Yiald outside default iimits.

LT - Result is less than Requested MDC. greater than sample specific MDC.

SQ - Spectral quality prevents accurate quantitation.

SI - Nuclide identlication and/or quantitation is tentative.

$T$ - Nuctide identification is tentative

$R$ - Nuclide has exceeded B halfives.
Abbreviations:

TPU - Total Propagated Uncertainty (see PAI SOP 743)

MDC - Minimum Detectable Concentration (see PAI SOP 709)

Data Package ID: GSS0305155-1 


\title{
Gamma Spectroscopy Results
}

\section{Method PAI 713R8}

\author{
Sample Duplicate Results
}

Client Name: Bechtel Nevada

Client Project Name: CAU 523

Client Project Number: V1979
Page: 3 of 6

Reported on: Thursday, May 29, 2003 10:41:50

Laboratory Name: Paragon Analytics, Inc.

PAl Work Order: 0305155
Field ID: 202228-1

Lab ID:0305155-3-D1
Sample Matrix: Solid

Date Prepared: 28-May-03

Prep SOP: PAI 739R6

Prep Batch: GS02053
Date Collected: 20-May-03

Date Analyzed: 28-May-03

Analytical SOP: PAl 713R8

Spectrum Code: 030691D02A
Final Allquot: 258.0

Aliquot Units: $\mathrm{g}$

Report Basis: Dry Weight Count Time (min.): 30

\begin{tabular}{|c|c|c|c|c|}
\hline Target Nuclide & Result $+/-2$ s TPU & MDC & $\begin{array}{c}\text { Reporting } \\
\text { Units }\end{array}$ & Lab Qualifier \\
\hline Ac-228 & $0.21+/-0.29$ & 0.49 & $\mathrm{pCi} / \mathrm{g}$ & U \\
\hline Am-241 & $-0.15+/-0.40$ & 0.79 & $\mathrm{pCi} / \mathrm{g}$ & $\mathrm{U}$ \\
\hline $\mathrm{Ce}-144$ & $0.15+/-0.28$ & 0.48 & $\mathrm{pCi} / \mathrm{g}$ & $U$ \\
\hline $\mathrm{Co}-60$ & $0.026+1-0.068$ & 0.13 & $\mathrm{pCi} / \mathrm{g}$ & $U$ \\
\hline Cs-134 & $-0.006+/-0.054$ & 0.11 & $\mathrm{pCi} / \mathrm{g}$ & $U$ \\
\hline Cs-137 & $0.007+/-0.060$ & 0.12 & $\mathrm{pCi} / \mathrm{g}$ & $\mathrm{U}$ \\
\hline Eu-152 & $0.15+/-0.25$ & 0.43 & $\mathrm{pCi} / \mathrm{g}$ & $\mathrm{U}$ \\
\hline Eu-154 & $-0.36+/-0.36$ & 0.84 & $\mathrm{pCi} / \mathrm{g}$ & $\mathrm{U}$ \\
\hline Eu-155 & $0.00+/-0.15$ & 0.28 & $\mathrm{pCi} / \mathrm{g}$ & $U$ \\
\hline$K-40$ & $4.8+/-1.7$ & 1.2 & $\mathrm{pCi} / \mathrm{g}$ & \\
\hline $\mathrm{Pb}-212$ & $0.30+/-0.15$ & 0.21 & $\mathrm{pCi} / \mathrm{g}$ & \\
\hline $\mathrm{Pb}-214$ & $0.28+/-0.15$ & 0.26 & $\mathrm{pCi} / \mathrm{g}$ & \\
\hline$P m-144$ & $-0.069+1-0.071$ & 0.15 & $\mathrm{pCi} / \mathrm{g}$ & $\bar{U}$ \\
\hline$P m-146$ & $0.021+/-0.059$ & 0.11 & $\mathrm{pCi} / \mathrm{g}$ & $U$ \\
\hline Ru-106 & $0.23+1-0.44$ & 0.76 & $\mathrm{pCi} / \mathrm{g}$ & U \\
\hline Sb-125 & $-0.02+/-0.13$ & 0.26 & $\mathrm{pCi} / \mathrm{g}$ & $\mathrm{U}$ \\
\hline Th-234 & $0.33+1-0.93$ & 1.6 & $\mathrm{pCi} / \mathrm{g}$ & U \\
\hline U-235 & $-0.04+1-0.30$ & 0.55 & $\mathrm{pCi} / \mathrm{g}$ & $\mathrm{U}$ \\
\hline$Y-88$ & $-0.006+/-0.077$ & 0.15 & $\mathrm{pCi} / \mathrm{g}$ & $\mathrm{U}$ \\
\hline
\end{tabular}

Datı Package ID: GSS0305155-1 


\section{Gamma Spectroscopy Results}

\section{Method PAI 713R8}

\section{Sample Duplicate Results}

Client Name: Bechtel Nevada

Client Project Name: CAU 523

Page: 4 of 6

Reported on: Thursday, May 29, 2003

$$
\text { 10:41:50 }
$$

Laboratory Name: Paragon Analytics, Inc.

PAI Work Order: 0305155

Date Collected: 20-May-03

Date Analyzed: 28-May-03

Analytical SOP: PAI 713R8

Spectrum Code: 030691D02A
Final Aliquot: 258.0

Aliquot Units: 9

Report Basis: Dry Weight Count Time (min.): 30
Date Prepared: 28-May-03

Prep SOP: PAI 739R6

Prep Batch: GS02053

\section{MDC}

Reporting Units

\section{Lab Qualifier}

\section{Comments:}

\section{Qunlifiers/Flags:}

$U$ - Result is less than the sample specific MDC or less than the associated TPU.

Y1 - Chemical Yield is in control at 100-110\%. Quantitative yield is assumed.

Y2 - Chemical Yield outside default limits.

- Duplicate DER not within control limits.

LT - Result is less then Requested MOC, greater than semple speclfic MDC.

SQ - Spectrai quality prevents accurate quantitation.

SI - Nuclide identification and/or quantitation is tentative.

TI - Nuclide identification is lentative.

$R$ - Nuclide has exceeded 8 halfives.
Abbreviations:

TPU - Total Propagated Uncertainty (see PAI SOP 743)

MDC - Minimum Detectable Concentration (see PAI SOP 709)

Data Package ID: GSS0305155-1 


\section{Gamma Spectroscopy Results}

\section{Method PAI 713R8}

\section{Sample Results}

Client Name: Bechtel Nevada

Client Project Name: CAU 523

Client Project Number: V1979
Page: 7 of 22

Reported on: Thursday, May 29, 2003

10:41:47

Laboratory Name: Paragon Analytics, Inc.

PAI Work Order: 0305155
Fieid ID:202228-2

Lab ID:0305155-4
Sample Matrix: Soil

Date Prepared: 28-May-03

Prep SOP: PAI $739 R 6$

Prep Batch: GSO2051
Date Collected: 20-May-03

Date Analyzed: 28-May-03

Analytical SOP: PAI 713R8

Spectrum Code: 030638D07A
Final Aliquot: $375.8 \mathrm{~g}$

Report Basis: Dry Weight

Count Time (min.): 30

Lbrary: LNG_GAM-A-00

\begin{tabular}{|c|c|c|c|c|}
\hline Target Nuclide & Result $+/-2$ s TPU & MDC & $\begin{array}{c}\text { Reporting } \\
\text { Units }\end{array}$ & Lab Qualifier \\
\hline AC-228 & $2.18+/-0.54$ & 0.55 & $\mathrm{pCi} / \mathrm{g}$ & \\
\hline Am-241 & $0.00+/-0.14$ & 0.24 & $\mathrm{pCi} / \mathrm{g}$ & $U$ \\
\hline $\mathrm{Bi}-212$ & $2.1+1-1.3$ & 1.9 & $\mathrm{pCi} / \mathrm{g}$ & \\
\hline $\mathrm{Bi}-214$ & $1.00+/-0.31$ & 0.29 & $\mathrm{pCi} / \mathrm{g}$ & \\
\hline Cd-109 & $5.4+/-1.9$ & 2.4 & $\mathrm{pCi} / \mathrm{g}$ & SI \\
\hline $\mathrm{Ce}-144$ & $-0.38+1-0.39$ & 0.72 & $\mathrm{pCi} / \mathrm{g}$ & U \\
\hline $\mathrm{Co}-60$ & $-0.010+1-0.100$ & 0.19 & $\mathrm{pCi} / \mathrm{g}$ & $\mathrm{U}$ \\
\hline$C_{s-134}$ & $0.036+/-0.087$ & 0.15 & $\mathrm{pCi} / \mathrm{g}$ & $\mathrm{U}$ \\
\hline Cs-137 & $0.032+/-0.078$ & 0.14 & $\mathrm{pCl} / \mathrm{g}$ & $U$ \\
\hline Eu-152 & $0.10+/-0.42$ & 0.79 & $\mathrm{pCi} / \mathrm{g}$ & $\mathrm{U}$ \\
\hline Eu-154 & $-0.30+1-0.57$ & 1.1 & $\mathrm{pCi} / \mathrm{g}$ & $U$ \\
\hline$E u-155$ & $0.24+1-0.20$ & 0.32 & $\mathrm{pCi} / \mathrm{g}$ & $U$ \\
\hline$K-40$ & $36.8+/-7.2$ & 1.7 & $\mathrm{pCi} / \mathrm{g}$ & \\
\hline $\mathrm{Pb}-212$ & $2.58+/-0.50$ & 0.18 & $\mathrm{pCi} / \mathrm{g}$ & \\
\hline $\mathrm{Pb}-214$ & $1.13+/-0.27$ & 0.26 & $\mathrm{pCi} / \mathrm{g}$ & \\
\hline$P m-144$ & $0.000+/-0.087$ & 0.16 & $\mathrm{pCi} / \mathrm{g}$ & $\mathrm{U}$ \\
\hline Pm-146 & $-0.055+/ \cdot 0.099$ & 0.19 & $\mathrm{pCl} / \mathrm{g}$ & $\mathrm{U}$ \\
\hline Ru-106 & $-0.37+1-0.73$ & 1.4 & $\mathrm{pCi} / \mathrm{g}$ & $\mathrm{U}$ \\
\hline Sb-125 & $-0.02+/-0.18$ & 0.34 & $\mathrm{pCi} / \mathrm{g}$ & $\mathrm{U}$ \\
\hline Th-234 & $1.55+/-0.82$ & 1.6 & $\mathrm{pCi} / \mathrm{g}$ & $\underline{U}$ \\
\hline $71-208$ & $0.83+/-0.20$ & 0.14 & $\mathrm{pCi} / \mathrm{g}$ & \\
\hline U-235 & $-0.20+/-0.41$ & 0.74 & $\mathrm{pCi} / \mathrm{g}$ & U \\
\hline$Y-88$ & $0.006+1-0.089$ & 0.17 & $\mathrm{pCi} / \mathrm{g}$ & $U$ \\
\hline
\end{tabular}

Data Package ID: GSS0305155-1 


\section{Gamma Spectroscopy Results}

\section{Method PAI 713R8}

\section{Sample Results}

Client Name: Bechtel Nevada

Client Project Name: CAU 523

Client Project Number: V1979
Page: 8 of 22

Reported on: Thursday, May 29, 2003

10:41:47

Laboratory Name: Paragon Analytics, Inc.

PAI Work Order: 0305155
Field ID:202228-2

Lab ID:0305155-4
Sample Matrix: Soil

Date Prepared: 28-May-03

Prep SOP: PAI 739R6

Prep Batch: GS02051
Date Collected: 20-May-03

Date Analyzed: 28-May-03

Analytical SOP: PAI 713R8

Spectrum Code: 030638D07A
Final Allquot: $\mathbf{3 7 5 . 8 \mathrm { g }}$

Report Basis: Dry Weight

Count Time (min.): 30

Library: LNG_GAM-A-00

\begin{tabular}{|l|l|l|l|l|}
\hline Target Nuclide & Result +/- 2 s TPU & MDC & $\begin{array}{c}\text { Reporting } \\
\text { Units }\end{array}$ & Lab Qualifier \\
\hline
\end{tabular}

\section{Comments:}

\section{Quallfier:/Fiags:}

$U$ - Result is less than the sample specific MDC or less than the associated TPU.

Y1 - Chemical Yield is in control at 100-110\%. Quantitative Yield is assumed.

Y2 - Chemical Yield outside default limits.

LT - Result is less than Requested MDC. greater than sample specific MDC.

SQ - Spectral quality prevents accurate quantitation.

S1 - Nuctide idenlification and/or quanttation is tentative.

TI - Nuclide identfication is tentative.

$R$ - Nuctide has exceeded 8 halfives.
Abbreviations:

TPU - Total Propagated Uncertainty (soe PAI SOP 743)

MDC - Minimum Detectable Concentration (see PAI SOP 709)

Data Package ID: GSS0305155-1 


\section{Gamma Spectroscopy Results}

\section{Method PAI 713R8}

\section{Sample Results}

Client Name: Bechtel Nevada

Cllent Project Name: CAU 523

Client Project Number: V1979
Page: 9 of 22

Reported on: Thursday, May 29, 2003 10:41:48

Laboratory Name: Paragon Analytics, Inc.

PAl Work Order: 0305155
Field tD:202228-3

Lab ID:0305155-5
Sample Matrix: Soil

Date Prepared: 28-May-03

Prep SOP: PAI 739R6

Prep Batch: GS02051
Date Collected: 20-May-03

Date Analyzed: 28-May-03

Analytical SOP: PAI 713R8

Spectrum Code: 030741D08A
Final Aliquot: $366.4 \mathrm{~g}$

Report Basis: Dry Weight

Count Time (min.): 30

Library: LNG_GAM-A-00

\begin{tabular}{|c|c|c|c|c|}
\hline Target Nuclide & Result +1- 2 s TPU & MDC & $\begin{array}{c}\text { Reporting } \\
\text { Units }\end{array}$ & Lab Qualifier \\
\hline Ac-228 & $2.48+/-0.57$ & 0.54 & $\mathrm{pCi} / \mathrm{g}$ & \\
\hline$A m-241$ & $-0.18+/-0.16$ & 0.29 & $\mathrm{pCi} / \mathrm{g}$ & $U$ \\
\hline $\mathrm{Bi}-212$ & $3.1+/-1.6$ & 2.0 & $\mathrm{pCi} / \mathrm{g}$ & \\
\hline $\mathrm{Bi}-214$ & $1.14+/-0.33$ & 0.32 & $\mathrm{pCi} / \mathrm{g}$ & \\
\hline Cd-109 & $4.4+/-1.8$ & 2.4 & $\mathrm{pCi} / \mathrm{g}$ & $\mathrm{SI}$ \\
\hline $\mathrm{Ce}-144$ & $0.14+/-0.42$ & 0.71 & $\mathrm{pCl} / \mathrm{g}$ & U \\
\hline Co-60 & $-0.032+1-0.095$ & 0.19 & $\mathrm{pCi} / \mathrm{g}$ & $u$ \\
\hline Cs-134 & $0.000+/-0.073$ & 0.13 & $\mathrm{pCi} / \mathrm{g}$ & $u$ \\
\hline Cs-137 & $0.048+/-0.087$ & 0.15 & $\mathrm{pCi} / \mathrm{g}$ & $U$ \\
\hline Eu-152 & $0.00+1-0.36$ & 0.70 & $\mathrm{pCi} / \mathrm{g}$ & U \\
\hline Eu-154 & $-0.39+1-0.46$ & 0.94 & $\mathrm{pCi} / \mathrm{g}$ & $u$ \\
\hline Eu-155 & $0.10+1-0.23$ & 0.38 & $\mathrm{pCi} / \mathrm{g}$ & U \\
\hline $\mathrm{K}-40$ & $34.0+1-6.6$ & 1.7 & $\mathrm{pCi} / \mathrm{g}$ & \\
\hline $\mathrm{Pb}-212$ & $2.63+/-0.50$ & 0.22 & $\mathrm{pCi} / \mathrm{g}$ & \\
\hline $\mathrm{Pb}-214$ & $1.24+/-0.29$ & 0.25 & $\mathrm{pCi} / \mathrm{g}$ & \\
\hline $\mathrm{Pm}-144$ & $-0.006+/-0.087$ & 0.16 & $\mathrm{pCi} / \mathrm{g}$ & $u$ \\
\hline $\mathrm{Pm}-146$ & $0.145+/-0.099$ & 0.14 & $\mathrm{pCi} / \mathrm{g}$ & $\mathrm{TI}$ \\
\hline Ru-106 & $0.18+/-0.65$ & 1.1 & $\mathrm{pCi} / \mathrm{g}$ & $U$ \\
\hline Sb-125 & $0.11+/-0.16$ & 0.31 & $\mathrm{pCi} / \mathrm{g}$ & $\bar{U}$ \\
\hline Th-234 & $2.1+/-1.1$ & 2.3 & $\mathrm{pCi} / \mathrm{g}$ & $u$ \\
\hline $\mathrm{TI}-208$ & $0.91+/-0.21$ & 0.13 & $\mathrm{pCi} / 9$ & \\
\hline U-235 & $0.16+/-0.41$ & 0.70 & $\mathrm{pCi} / \mathrm{g}$ & U \\
\hline$Y-88$ & $0.033+/-0.082$ & 0.14 & $\mathrm{pCi} / \mathrm{g}$ & U \\
\hline
\end{tabular}

Data Package ID: GSS0305155-1 


\section{Gamma Spectroscopy Results}

\section{Method PAI 713R8}

\section{Sample Results}

Client Name: Bechtel Nevada

Client Project Name: CAU $\mathbf{5 2 3}$

Client Project Number: V1979
Page: 10 of 22

Reported on: Thursday, May 29, 2003

10:41:48

Laboratory Name: Paragon Analytics, Inc.

PAI Work Order: 0305155
Field ID:202228-3

Lab ID:0305155-5
Sample Matrix: Soil

Date Prepared: 28-May-03

Prep SOP: PAI $739 R 6$

Prep Batch: GS02051
Date Collected: 20-May-03

Date Analyzed: 28-May-03

Analytical SOP: PAI 713R8

Spectrum Code: 030741D08A
Final Aliquot: $366.4 \mathrm{~g}$

Report Basis: Dry Weight

Count Time (min.): 30

Library: LNG_GAM-A-D0

Target Nuclide

Result +/- 2 s TPU

\section{MDC}

Reporting Units
Lab Qualifier

\section{Comments:}

\section{Qualifiers/Flags:}

U - Result is less than the sample specific MDC or less than the associated TPU.

$Y 1$ - Chemical Yield is in control at 100-110\%. Quantitative Yield is assumed.

Y2 - Chemical Yield outside defaull limits.

LT - Result is less than Requeated MDC, greater than sample specific MDC.

SQ - Spectral quality prevents accurate quantltation.

SI - Nuclide identification and/or quantitation is tentative.

TI - Nuclide identification is tentabve.

$R$ - Nuclide has exceeded 8 halfives.
Abbraviations:

TPU - Total Propagated Uncertainty (see PAI SOP 743)

MDC - Minimum Deleclable Concentration (see PAI SOP 709)

Data Package ID: GSSO305155-1 


\section{Gamma Spectroscopy Results}

\section{Method PAI 713R8}

\section{Sample Results}

Client Name: Bechtel Nevada Client Project Name: CAU 523

Client Project Number: V1979
Page: 11 of 22

Reported on: Thursday, May 29, 2003 10:41:48

Laboratory Name: Paragon Analytics, Inc.

PAI Work Order: 0305155
Field ID:202229-1

Lab ID:0305155-6
Sample Matrix: Soil

Date Prepared: 28-May-03

Prep SOP: PAI 739R6

Prep Batch: GS02051
Date Collected: 20-May-03

Date Analyzed: 28-May-03

Analytical SOP: PAI 713R8

Spectrum Code: 030631D06A
Final Aliquot: $367.8 \mathrm{~g}$

Report Basis: Dry Weight

Count Time (min.): 30

Library: LNG_GAM-A-00

\begin{tabular}{|c|c|c|c|c|}
\hline Target Nuclide & Result $+1-2$ s TPU & MDC & $\begin{array}{l}\text { Reporting } \\
\text { Units }\end{array}$ & Lab Qualifier \\
\hline AC-228 & $1.92+/-0.53$ & 0.85 & $\mathrm{pCi} / \mathrm{g}$ & \\
\hline$A m-241$ & $-0.08+1-0.41$ & 0.73 & $\mathrm{pCi} / \mathrm{g}$ & U \\
\hline $\mathrm{Bi}-214$ & $1.12+/-0.32$ & 0.29 & $\mathrm{pCl} / \mathrm{g}$ & \\
\hline Cd-109 & $4.3+1-2.3$ & 3.4 & $\mathrm{pCi} / 9$ & SI \\
\hline $\mathrm{Ce}-144$ & $0.09+1-0.4 .1$ & 0.70 & $\mathrm{pCi} / \mathrm{g}$ & $u$ \\
\hline Co-60 & $0.033+/-0.093$ & 0.16 & $\mathrm{pCi} / \mathrm{g}$ & $u$ \\
\hline Cs-134 & $0.034+/-0.079$ & 0.13 & $\mathrm{pCi} / \mathrm{g}$ & $u$ \\
\hline Cs-137 & $0.084+1-0.097$ & 0.16 & $\mathrm{pCi} / \mathrm{g}$ & $u$ \\
\hline Eu-152 & $0.09+/-0.45$ & 0.82 & $\mathrm{pCi} / \mathrm{g}$ & $U$ \\
\hline Eu-154 & $0.24+1-0.50$ & 0.86 & $\mathrm{pCi} / \mathrm{g}$ & $\bar{u}$ \\
\hline Eu-155 & $0.10+/-0.22$ & 0.38 & $\mathrm{pCi} / \mathrm{g}$ & $u$ \\
\hline $\mathrm{K}-40$ & $29.4+1-5.8$ & 2.3 & $\mathrm{pCi} / \mathrm{g}$ & \\
\hline $\mathrm{Pb}-212$ & $1.90+/-0.39$ & 0.25 & $\mathrm{pCi} / \mathrm{g}$ & \\
\hline $\mathrm{Pb}-214$ & $1.25+/-0.31$ & 0.36 & $\mathrm{pCi} / \mathrm{g}$ & \\
\hline $\mathrm{Pm}-144$ & $-0.027+/-0.097$ & 0.18 & - $\mathrm{pCi} / \mathrm{g}$ & $u$ \\
\hline $\mathrm{Pm}-146$ & $-0.067+/-0.096$ & 0.18 & $\mathrm{pCi} / \mathrm{g}$ & u \\
\hline Ru-106 & $-0.48+1-0.72$ & 1.4 & $\mathrm{pCi} / \mathrm{g}$ & $u$ \\
\hline $\mathrm{Sb}-125$ & $0.18+/-0.20$ & 0.36 & $\mathrm{pCi} / \mathrm{g}$ & $u$ \\
\hline Th-234 & $2.3+/-1.4$ & 2.1 & $\mathrm{pCi} / \mathrm{g}$ & $T 1$ \\
\hline $\mathrm{TI}-208$ & $0.50+/-0.16$ & 0.16 & $\mathrm{pCi} / \mathrm{g}$ & \\
\hline U-235 & $-0.37+/-0.40$ & 0.73 & $\mathrm{pCi} / \mathrm{g}$ & $u$ \\
\hline $\mathrm{Y}-88$ & $-0.078+1-0.087$ & 0.18 & $\mathrm{pCi} / \mathrm{g}$ & $u$ \\
\hline
\end{tabular}

Data Paskage ID: GSS0305155-1 


\section{Gamma Spectroscopy Results}

\section{Method PAI 713R8}

\section{Sample Results}

Client Name: Bechtel Nevada

Client Project Name: CAU 523

Client Project Number: V1979
Page: 12 of 22

Reported on: Thursday, May 29, 2003 10:41:48

Laboratory Name: Paragon Analytics, Inc.

PAl Work Order: 0305155
Field ID:202229-1

Lab ID:0305155-6
Sample Matrix: Soil

Date Prepared: 28-May-03

Prep SOP: PAI 739R6

Prep Batch: GS02051
Date Collected: 20-May-03

Date Analyzed: 28-May-03

Analytical SOP: PAI 713R8

Spectrum Code: 030631D06A
Final Aliquot: $367.8 \mathrm{~g}$

Report Basis: Dry Weight

Count Time (min.): 30

Library: LNG_GAM-A-00

Target Nuclide

Result +1- 2 s TPU

MDC

Reporting

Lab Qualifier Units

\section{Comments:}

\section{Qualfiers/Flags:}

$U$ - Result is less than the sample specific MDC or less than the associated TPU

Y1 - Chemical Yield is in control at 100-110\%. Quantitative Yield is assumed.

Y2 - Chemical Yield outside default limits.

LT - Result is less than Requested MDC, greater than sample specific MDC.

SQ - Spectral quality prevents accurate quantitation.

SI - Nuclide identification and/or quantitation is tentative.

$\mathrm{Tf}$ - Nuclide identification is lentalive.

$R$ - Nuctide has exceeded 8 hallives.
Abbreviations:

TPU - Total Propagated Uncertainty (see PAI SOP 743)

MDC - Minimum Detectable Concentration (see PAI SOP 709) 


\section{Gamma Spectroscopy Results}

\section{Method PAI 713R8}

\section{Sample Results}

- Client Name: Bechtel Nevada

Client Project Name: CAU 523

Client Project Number: V1979
Page: 13 of 22

Reported on: Thursday, May 29, 2003

10:41:48

Laboratory Name: Paragon Analytics, Inc.

PAI Work Order: 0305155
Field ID: $202227-1$

Lab ID:0305155-7
Sample Matrix: Soil

Date Prepared: 28-May-03

Prep SOP: PAI $739 R 6$

Prep Batch: GS02051
Date Collected: 20-May-03

Date Analyzed: 28-May-03

Analytical SOP: PAI 713R8

Spectrum Code: 030639D07A
Final Aliquot: $339.9 \mathrm{~g}$

Report Basis: Dry Weight Count Time (min.): 30

Library: LNG_GAM-A-00

\begin{tabular}{|c|c|c|c|c|}
\hline Target Nuclide & Result +1- 2 s TPU & MDC & $\begin{array}{c}\text { Reporting } \\
\text { Units }\end{array}$ & Lab Qualifier \\
\hline Ac-228 & $1.80+/-0.50$ & 0.70 & $\mathrm{pCi} / 9$ & \\
\hline Am-241 & $0.01+/-0.13$ & 0.24 & $\mathrm{pCi} / \mathrm{g}$ & U \\
\hline $\mathrm{Bi}-214$ & $1.21+/-0.34$ & 0.30 & $\mathrm{pCi} / \mathrm{g}$ & \\
\hline Cd-109 & $6.4+/-2.2$ & 2.8 & $\mathrm{pCi} / \mathrm{g}$ & SI \\
\hline $\mathrm{Ce}-144$ & $-0.01+1-0.40$ & 0.70 & $\mathrm{pCi} / \mathrm{g}$ & $u$ \\
\hline Co-60 & $0.00+/-0.11$ & 0.21 & $\mathrm{pCi} / \mathrm{g}$ & u \\
\hline Cs-134 & $0.023+/-0.078$ & 0.14 & $\mathrm{pCi} / \mathrm{g}$ & U \\
\hline Cs-137 & $0.22+/-0.12$ & 0.14 & $\mathrm{pCi} / \mathrm{g}$ & \\
\hline Eu-152 & $0.44+/-0.45$ & 0.66 & $\mathrm{pCi} / \mathrm{g}$ & $u$ \\
\hline Eu-154 & $-0.19+/-0.43$ & 0.90 & $\mathrm{pCi} / \mathrm{g}$ & $u$ \\
\hline Eu-155 & $0.24+/-0.20$ & 0.31 & $\mathrm{pCi} / \mathrm{g}$ & $U$ \\
\hline$K-40$ & $24.8+/-5.3$ & 1.9 & $\mathrm{pCi} / \mathrm{g}$ & \\
\hline $\mathrm{Pb}-212$ & $2.47+/-0.49$ & 0.22 & $\mathrm{pCi} / \mathrm{g}$ & \\
\hline $\mathrm{Pb}-214$ & $1.25+/-0.31$ & 0.33 & $\mathrm{pCi} / \mathrm{g}$ & \\
\hline $\mathrm{Pm}-144$ & $-0.032+1-0.088$ & 0.17 & $\mathrm{pCi} / \mathrm{g}$ & $u$ \\
\hline $\mathrm{Pm}-146$ & $0.015+1-0.090$ & 0.16 & $\mathrm{pCi} / \mathrm{g}$ & U \\
\hline Ru-106 & $-0.23+1-0.76$ & 1.5 & $\mathrm{pCi} / \mathrm{g}$ & $u$ \\
\hline Sb-125 & $0.01+1-0.20$ & 0.40 & $\mathrm{pCi} / \mathrm{g}$ & $u$ \\
\hline Th-234 & $3.2+/-1.4$ & 1.9 & $\mathrm{pCi} / \mathrm{g}$ & $\mathrm{TI}$ \\
\hline TH-208 & $0.73+/-0.20$ & 0.17 & $\mathrm{pCi} / \mathrm{g}$ & \\
\hline $\mathrm{U}-235$ & $0.12+1-0.40$ & 0.68 & $\mathrm{pCi} / 9$ & $u$ \\
\hline$Y-88$ & $0.089+/-0.096$ & 0.15 & $\mathrm{pCi} / \mathrm{g}$ & $\bar{U}$ \\
\hline
\end{tabular}

Data Package ID: GSS0305155-1 


\section{Gamma Spectroscopy Results}

\section{Method PAI 713R8}

\section{Sample Results}

Client Name: Bechtel Nevada

Client Project Name: CAU 523
Page: 14 of 22

Reported on: Thursday, May 29, 2003 10:41:48

Laboratory Name: Paragon Analytics, Inc.

PAI Work Order: 0305155
Final Aliquot: $339.9 \mathrm{~g}$

Report Basis: Dry Weight

Date Prepared: 28-May-03

Date Collected: 20-May-03

Date Analyzed: 28-May-03

Analytical SOP: PAI 713R8

Count Time (min.): 30

Spectrum Code: 030639D07A

Library: LNG_GAM-A-00

\begin{tabular}{|l|l|l|l|l|}
\hline Target Nuclide & Result $+1-2$ s TPU & MDC & $\begin{array}{c}\text { Reporting } \\
\text { Units }\end{array}$ & Lab Qualifier \\
\hline
\end{tabular}

\section{Comments:}

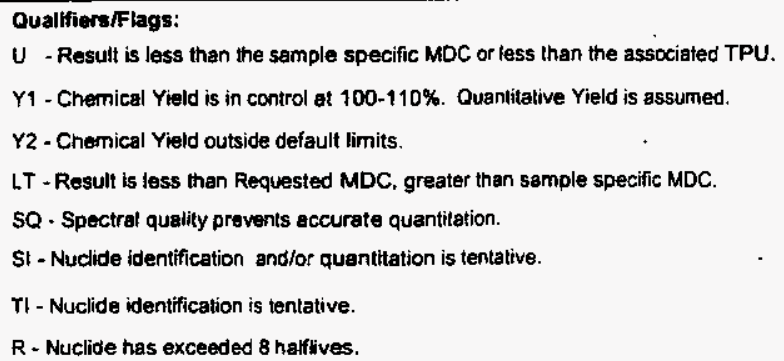

\section{Abbreviations:}

TPU - Total Propagated Uncertainty (see PAI SOP 743)

MDC - Minimum Detectable Concentration (see PAI SOP 709)

\section{Paragon Analytics Inc.}




\section{Gamma Spectroscopy Results}

\section{Method PAI 713R8}

\section{Sample Results}

Client Name: Bechtel Nevada

Client Project Name: CAU 523

Client Project Number: V1979
Page: 15 of 22

Reported on: Thursday, May 29, 2003 10:41:44

Laboratory Name: Paragon Analytics, Inc.

PAl Work Order: 0305155
Field ID:202227-2

Lab ID:0305155-8
Sample Matrix: Soil

Date Prepared: 28-May-03

Prep SOP: PAI 739R6

Prep Batch: GS02052
Date Collected: 20-May-03

Date Analyzed: 28-May-03

Analytical SOP: PAI $713 R 8$

Spectrum Code: 030263004A
Final Aliquot: $82.70 \mathrm{~g}$

Report Basis: Dry Weight

Count Time (min.): 30

Library: LNG_GAM-A-00

\begin{tabular}{|c|c|c|c|c|}
\hline Target Nuclide & Result +/- 2 s TPU & MDC & $\begin{array}{c}\text { Reporting } \\
\text { Units }\end{array}$ & Lab Qualifier \\
\hline Ac-228 & $2.19+1-0.68$ & 0.98 & $\mathrm{pCi} / \mathrm{g}$ & \\
\hline$A m-241$ & $-0.06+/-0.51$ & 0.92 & $\mathrm{pCi} / \mathrm{g}$ & $\mathrm{U}$ \\
\hline $\mathrm{Bi}-214$ & $0.99+1-0.37$ & 0.39 & $\mathrm{pCi} / \mathrm{g}$ & \\
\hline Ce-144 & $0.20+/-0.51$ & 0.88 & $\mathrm{pCi} / \mathrm{g}$ & $\vec{U}$ \\
\hline $\mathrm{Co-60}$ & $-0.04+/-0.11$ & 0.23 & $\mathrm{pCi} / \mathrm{g}$ & $u$ \\
\hline Cs-134 & $-0.01+/-0.11$ & 0.21 & $\overline{\mathrm{pCi} / \mathrm{g}}$ & $\mathrm{U}$ \\
\hline Cs-137 & $0.34+\% \quad 0.17$ & 0.21 & $\mathrm{pCi} / \mathrm{g}$ & \\
\hline Eu-152 & $0.05+1 / 0.49$ & 1.00 & $\mathrm{pCi} / \mathrm{g}$ & $\bar{U}$ \\
\hline Eu-154 & $-0.12+/-0.52$ & 1.1 & $\mathrm{pCi} / \mathrm{g}$ & $u$ \\
\hline Eu-155 & $-0.13+/-0.29$ & 0.54 & $\mathrm{pCi} / \mathrm{g}$ & $u$ \\
\hline$K-40$ & $21.5+/-5.3$ & 2.7 & $\mathrm{pCi} / \mathrm{g}$ & \\
\hline $\mathrm{Pb}-212$ & $1.68+/-0.41$ & 0.34 & $\mathrm{pCi} / \mathrm{g}$ & \\
\hline $\mathrm{Pb}-214$ & $1.24+1-0.33$ & 0.41 & $\mathrm{pCi} / \mathrm{g}$ & \\
\hline $\mathrm{Pm}-144$ & $-0.07+1-0.13$ & 0.25 & $\mathrm{pCi} / \mathrm{g}$ & $u$ \\
\hline$P m-146$ & $0.09+1-0.14$ & 0.23 & $\mathrm{pCi} / \mathrm{g}$ & $u$ \\
\hline Ru-106 & $-0.60+/-0.99$ & 2.0 & $\mathrm{pCi} / \mathrm{g}$ & $u$ \\
\hline Sb-125 & $0.15+1-0.29$ & 0.56 & $\mathrm{pCi} / \mathrm{g}$ & U \\
\hline Th-234 & $2.5+1-1.9$ & 2.8 & $\mathrm{pCi} / \mathrm{g}$ & $u$ \\
\hline $\mathrm{TI}-208$ & $0.63+/-0.21$ & 0.18 & $\mathrm{pCi} / \mathrm{g}$ & \\
\hline U-235 & $-0.28+/-0.47$ & 0.88 & $\mathrm{pCi} / \mathrm{g}$ & $u$ \\
\hline$Y-88$ & $0.03+/-0.13$ & 0.24 & $\mathrm{pCi} / \mathrm{g}$ & $u$ \\
\hline
\end{tabular}

Data Package ID: GSS0305155-1 


\section{Gamma Spectroscopy Results}

\section{Method PAI 713R8}

\section{Sample Results}

Client Name: Bechtel Nevada

Client Project Name: CAU 523

Client Project Number: V1979
Page: 16 of 22

Reported on: Thursday, May 29, 2003

10:41:45

Laboratory Name: Paragon Analytics, Inc.

PAI Work Order: 0305155
Field ID:202227-2

Lab 1D:0305155-8
Sample Matrix: Soil

Date Prepared: 28-May-03

Prep SOP: PAI 739R6

Prep Batch: GS02052
Date Collected: 20-May-03

Date Analyzed: 28-May-03

Analytical SOP: PAI 713R8

Spectrum Code: 030263D04A
Final Aliquot: $82.70 \mathrm{~g}$

Report Basis: Dry Weight

Count Time (min.): 30

Library: LNG_GAM-A-00

\begin{tabular}{|l|l|l|l|l|}
\hline Target Nuclide & Result +1.2 s TPU & MDC & $\begin{array}{c}\text { Reporting } \\
\text { Units }\end{array}$ & Lab Qualifier \\
\hline
\end{tabular}

\section{Comments:}

Qualifiers/Flags:

U - Result is less than the sample specific MDC or less than the associated TPU

Y1 - Chemical Yield is in control at 100-110\%. Quantitalive Yield is assumed

Y2 - Chemical Yield outside defaull limits.

LT - Resull is less than Requested MDC. greater than sample specific MDC.

SQ - Spectral quality prevents accurate quantitation.

Si - Nuclide identification andor quantitation is tentative

$\mathrm{TI}$ - Nuclide identification is tentative.

$R$ - Nuctide has exceeded $\theta$ halflives.
Abbreviations:

TPU - Total Propagaled Uncertainty (see PAI SOP 743)

MOC - Minimum Detectable Concentration (see PAI SOP 709)

Data Package ID: GSS0305155-1 


\title{
Gamma Spectroscopy Results
}

\author{
Method PAI 713R8
}

\section{Sample Duplicate Results}

Client Name: Bechtel Nevada

Client Project Name: CAU 523

Client Project Number: V1979
Page: 5 of 6

Reported on: Thursday, May 29, 2003 10:41:45

Laboratory Name: Paragon Analytics, Inc.

PAl Work Order: 0305155
Field ID:202227-2

Lab ID:0305155-8-D1
Sample Matrix: Soil

Date Prepared: 28-May-03

Prep SOP: PAI 739R6

Prep Batch: GS02052
Date Collected: 20-May-03

Date Analyzed: 28-May-03

Analytical SOP: PAI 713R8

Spectrum Code: 030654009A
Final Aliquot: 76.50

Aliquot Units: $\mathrm{g}$

Report Basis: Dry Weight Count Time (min.): 30

\begin{tabular}{|c|c|c|c|c|}
\hline Target Nuclide & Result +1- 2 s TPU & MDC & $\begin{array}{c}\text { Reporting } \\
\text { Units }\end{array}$ & Lab Qualiffer \\
\hline AC-228 & $1.46+1-0.65$ & 0.88 & $\mathrm{pCi} / \mathrm{g}$ & \\
\hline$A m-241$ & $0.49+/-0.97$ & 1.6 & $\mathrm{pCi} / \mathrm{g}$ & $\mathrm{U}$ \\
\hline $\mathrm{Bi}-214$ & $1.14+/-0.50$ & 0.59 & $\mathrm{pCi} / \mathrm{g}$ & \\
\hline $\mathrm{Ce}-144$ & $0.23+1-0.62$ & 1.1 & $\mathrm{pCi} / \mathrm{g}$ & U \\
\hline $\mathrm{Co}-60$ & $0.13+/ .0 .16$ & 0.26 & $\mathrm{pCi} / \mathrm{g}$ & $U$ \\
\hline Cs-134 & $-0.07+/-0.24$ & 0.45 & $\mathrm{pCi} / \mathrm{g}$ & $U$ \\
\hline Cs-137 & $0.12+/-0.21$ & 0.36 & $\mathrm{pCi} / \mathrm{g}$ & U \\
\hline Eu-152 & $-0.20+1-0.83$ & 1.8 & $\mathrm{pCi} / \mathrm{g}$ & $U$ \\
\hline Eu-154 & $-0.2+/-1.0$ & 2.0 & $\mathrm{pCi} / \mathrm{g}$ & $U$ \\
\hline Eu-155 & $-0.14+1-0.42$ & 0.76 & $\mathrm{pCi} / \mathrm{g}$ & U \\
\hline$K-40$ & $27.0+1-7.2$ & 4.7 & $\mathrm{pCi} / \mathrm{g}$ & \\
\hline $\mathrm{Pb}-212$ & $1.77+1-0.48$ & 0.45 & $\mathrm{pCi} / \mathrm{g}$ & \\
\hline $\mathrm{Pb}-214$ & $1.12+/-0.40$ & 0.55 & $\mathrm{pCi} / \mathrm{g}$ & \\
\hline$P m-144$ & $0.00+1-0.17$ & 0.31 & $\mathrm{pCi} / \mathrm{g}$ & U \\
\hline $\mathrm{Pm}-146$ & $0.01+/-0.17$ & 0.31 & $\mathrm{pCi} / \mathrm{g}$ & U \\
\hline Ru-106 & $-0.5+/-1.4$ & 2.7 & $\mathrm{pCi} / \mathrm{g}$ & U \\
\hline Sb-125 & $-0.14+/-0.40$ & 0.76 & $\mathrm{pCi} / \mathrm{g}$ & U \\
\hline Th-234 & $1.1+/-2.5$ & 4.2 & $\mathrm{pCi} / \mathrm{g}$ & U \\
\hline TI-208 & $0.45+/-0.27$ & 0.38 & $\mathrm{pCi} / \mathrm{g}$ & \\
\hline U-235 & $0.19+1-0.60$ & 1.0 & $\mathrm{pCi} / \mathrm{g}$ & U \\
\hline$Y-88$ & $-0.11+/-0.16$ & 0.36 & $\mathrm{pCi} / \mathrm{g}$ & $U$ \\
\hline
\end{tabular}

Data Package ID: GSS\$0305155-1

Paragon Analytics Inc. 


\section{Gamma Spectroscopy Results}

\section{Method PAI 713R8}

\section{Sample Duplicate Results}

Client Name: Bechtel Nevada

Client Project Name: CAU 523 Client Project Number: V1979
Page: 6 of 6

Reported on: Thursday, May 29, 2003

$$
\text { 10:41:45 }
$$

Laboratory Name: Paragon Analytics, Inc. PAI Work Order: 0305155
Field ID:202227-2

Lab 10:0305155-8-D1
Sampie Matrix: Soil

Date Prepared: 28-May-03

Prep SOP: PAI 739R6

Prep Batch: GS02052
Date Collected: 20-May-03

Date Analyzed: 28-May-03

Analytical SOP: PAI 713R8

Spectrum Code: 030654D09A
Final Aliquot: 76.50

Aliquot Units: $g$

Report Basis: Dry Weight Count Time (min.): 30

\begin{tabular}{|l|l|l|l|l|}
\hline Target Nuclide & Result +/- 2 s TPU & MDC & $\begin{array}{c}\text { Reporting } \\
\text { Units }\end{array}$ & Lab Qualifier \\
\hline
\end{tabular}

\section{Comments:}

\section{Quallifers/Flags:}

$U$ - Result is less than the sample specific MDC or less than the associated TPU

Y1 - Chemical Yield is in control at 100-110\%. Quantitalive yield is assumed.

Y2 - Chemical Yield outside default timits.

- - Duplicate DER not within control limits.

LT - Result is less than Requested MDC, greater than sample specific MDC.

SQ - Spectral quality prevents accurate quantitation.

SI - Nuclide identification and/or quantitation is tentative.

$T i$ - Nuclide identification is tentative.

$R$ - Nuclide has exceeded 8 halflives.
Abbreviations:

TPU - Total Propagated Uncertainty (see PAI SOP 743)

MDC - Minimum Detectable Concentration (see PAI SOP 709)

Data Package ID: GSS0305155-1 


\section{Gamma Spectroscopy Results}

\section{Method PAI 713R8}

\section{Sample Results}

Client Name: Bechtel Nevada Client Project Name: CAU 523 Client Project Number: V1979
Page: 17 of 22

Reported on: Thursday, May 29, 2003 10:41:45

Laboratory Name: Paragon Analytics, Inc.

PAI Work Order: 0305155
Field ID:202227-3

Lab ID:0305155-9
Sample Matrix: Soil Date Prepared: 28-May-03 Prep SOP: PAl 739R6 Prep Batch: GS02052
Date Collected: 20-May-03

Date Analyzed: 28-May-03

Analytical SOP: PAI 713R8

Spectrum Code: 030514D03A
Final Aliquot: $56.40 \mathrm{~g}$

Report Basis: Dry Weight Count Time (min.): 30

Library: LNG_GAM-A-00

\begin{tabular}{|c|c|c|c|c|}
\hline Target Nuclide & Result + 1.2 s TPU & MDC & $\begin{array}{l}\text { Reporting } \\
\text { Units }\end{array}$ & Lab Qualifier \\
\hline Ac-228 & $1.47+/-0.97$ & 1.3 & $\mathrm{pCl} / \mathrm{g}$ & $\mathrm{TI}$ \\
\hline Am-241 & $-0.05+1-0.90$ & 1.6 & $\mathrm{pCl} / \mathrm{g}$ & $\bar{U}$ \\
\hline $8 \mathrm{Bi}-214$ & $0.70+/-0.49$ & 0.70 & $\mathrm{pCl} / \mathrm{g}$ & \\
\hline $\mathrm{Ce}-144$ & $0.09+/-0.67$ & 1.2 & $\mathrm{pCi} / \mathrm{g}$ & $U$ \\
\hline Co-60 & $0.04+/-0.24$ & 0.45 & $\mathrm{pCl} / \mathrm{g}$ & $u$ \\
\hline Cs-134 & $0.10+/-0.18$ & 0.30 & $\mathrm{pCi} / 9$ & $U$ \\
\hline Cs-137 & $-0.05+/-0.21$ & $\overline{0.41}$ & $\mathrm{pCl} / \mathrm{g}$ & $\bar{U}$ \\
\hline Eu-152 & $0.49+/-0.80$ & 1.4 & $\mathrm{pCi} / \mathrm{g}$ & $u$ \\
\hline Eu-154 & $0.00+/-1.00$ & 2.0 & $\mathrm{pCi} / \mathrm{g}$ & $U$ \\
\hline Eu-155 & $0.10+/-0.42$ & 0.74 & $\mathrm{pCi} / \mathrm{g}$ & $U$ \\
\hline $\mathrm{K}-40$ & $17.7+/-5.5$ & 3.7 & $\mathrm{pCi} / \mathrm{g}$ & \\
\hline $\mathrm{Pb}-212$ & $1.41+/-0.46$ & 0.49 & $\mathrm{pCi} / \mathrm{g}$ & \\
\hline$P m-144$ & $-0.02+/-0.17$ & 0.32 & $\mathrm{pCi} / \mathrm{g}$ & U \\
\hline $\mathrm{Pm}-146$ & $0.10+/-0.21$ & 0.36 & $\mathrm{pCi} / \mathrm{g}$ & U \\
\hline Ru-106 & $-0.1+/-1.5$ & 2.9 & $\mathrm{pCi} / \mathrm{g}$ & $\mathrm{U}$ \\
\hline Sb-125 & $-0.48+/-0.43$ & 0.91 & $\mathrm{pCi} / \mathrm{g}$ & $U$ \\
\hline Th-234 & $1.4+/-2.5$ & 4.1 & $\mathrm{pCi} / \mathrm{g}$ & $\bar{U}$ \\
\hline$U-235$ & $0.17+/-0.74$ & 1.3 & $\mathrm{pCi} / \mathrm{g}$ & $\bar{U}$ \\
\hline$Y-88$ & $0.10+/-0.16$ & 0.28 & $\mathrm{pCi} / \mathrm{g}$ & 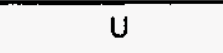 \\
\hline
\end{tabular}

Data Package ID: GSS0305155-1 


\section{Gamma Spectroscopy Results}

\section{Method PAI 713R8}

\section{Sample Results}

Client Name: Bechtel Nevada

Client Project Name: CAU 523

Client Project Number: V1979
Page: 18 of 22

Reported on: Thursday. May 29, 2003 10:41:45

Laboratory Name: Paragon Analytics, Inc.

PAI Work Order: 0305155
Field 10:202227-3

Lab ID:0305155-9
Sample Matrix: Soil

Date Prepared: 28-May-03

Prep SOP: PAI 739R6

Prep Batch: GS02052
Date Collected: 20-May-03

Date Analyzed: 28-May-03

Analytical SOP: PAI 713R8

Spectrum Code: 030514003A
Final Aliquot: $56.40 \mathrm{~g}$

Report Basis: Dry Weight

Count Time (min.): 30

Library: LNG_GAM-A-O0

\begin{tabular}{|c|c|c|c|c|}
\hline Target Nuclide & Result +/- 2 s TPU & MDC & $\begin{array}{c}\text { Reporting } \\
\text { Units }\end{array}$ & Lab Qualifier \\
\hline
\end{tabular}

\section{Comments:}

Qualifiers/Flegs:

$U$ - Result is less than the sample specific MDC or less than the associated TPU.

Y9-Chemical Yield is in control at 100-110\%. Quantitative Yietd is assumed.

Y2 - Chemical Yield outside detault limits.

LT - Result is less than Requested MDC, greater than sample specific MDC.

SQ - Spectral quality prevents accurale quantitation.

SI - Nuclide identification and/or quantilation is lentative.

$\mathrm{TI}$ - Nuclide identification is tentative.

$R$ - Nuclide has exceeded 8 hattlives.
Abbreviations:

TPU - Total Propagated Uncertainty (see PAI SOP 743)

MDC - Minimum Detectable Concentration (see PAI SOP 709)

Data Package ID: GSS0305155-1 


\section{Gamma Spectroscopy Results}

\section{Method PAI 713R8}

\section{Sample Results}

Client Name: Bechtel Nevada

Client Project Name: CAU 523

Client Project Number: V1979
Page: 19 of 22

Reported on: Thursday, May 29, 2003 10:41:46

Laboratory Name: Paragon Analytics, Inc.

PAI Work Order: 0305155
Field ID: 192231-1

Lab ID:0305155-10
Sample Matrix: Soil

Date Prepared: 28-May-03

Prep SOP: PAI 739R6

Prep Batch: GS02051
Date Collected: 20-May-03

Date Analyzed: 28-May-03

Analytical SOP: PAI 713R8

Spectrum Code: 030742D08A
Final Aliquot: $417.5 \mathrm{~g}$

Report Basis: Dry Weight Count Time (min.): 30

Library: LNG_GAM-A-00

\begin{tabular}{|c|c|c|c|c|}
\hline Target Nuclide & Result $+1-2$ s TPU & MDC & $\begin{array}{l}\text { Reporting } \\
\text { Units }\end{array}$ & Lab Qualifier \\
\hline Ac-228 & $2.24+/-0.50$ & 0.47 & $\mathrm{pCi} / \mathrm{g}$ & \\
\hline$A m-241$ & $0.03+/-0.13$ & 0.22 & $\mathrm{pCl} / \mathrm{g}$ & $U$ \\
\hline $\mathrm{Bi}-212$ & $2.0+/-1.1$ & 1.4 & $\mathrm{pCl} / \mathrm{g}$ & . \\
\hline $\mathrm{Bi}-214$ & $1.14+1-0.30$ & 0.25 & $\mathrm{pCl} / \mathrm{g}$ & \\
\hline Cd-109 & $4.8+1-1.8$ & 2.4 & $\mathrm{pCi} / \mathrm{g}$ & SI \\
\hline $\mathrm{Ce}-144$ & $-0.07+1-0.37$ & 0.66 & $\mathrm{pCi} / \mathrm{g}$ & $U$ \\
\hline $\mathrm{Co}-60$ & $0.000+1-0.075$ & 0.14 & $\mathrm{pCi} / \mathrm{g}$ & $U$ \\
\hline Cs-134 & $-0.023+/-0.055$ & 0.11 & $\mathrm{pCi} / \mathrm{g}$ & $U$ \\
\hline Cs-137 & $0.36+1-0.13$ & 0.16 & $\mathrm{pCl} / \mathrm{g}$ & \\
\hline Eu-152 & $0.00+/-0.36$ & 0.69 & $\mathrm{pCl} / \mathrm{g}$ & $u$ \\
\hline Eu-154 & $-0.16+/-0.43$ & 0.83 & $\mathrm{pCl} / \mathrm{g}$ & $u$ \\
\hline Eu-155 & $-0.11+/-0.20$ & 0.35 & $\mathrm{pCi} / \mathrm{g}$ & $\bar{U}$ \\
\hline$K-40$ & $26.2+1-5.1$ & 1.4 & $\mathrm{pCi} / \mathrm{g}$ & \\
\hline $\mathrm{Pb}-212$ & $2.18+1-0.42$ & 0.20 & $\mathrm{pCl} / \mathrm{g}$ & \\
\hline $\mathrm{Pb}-214$ & $1.38+1-0.30$ & 0.22 & $\mathrm{pCi} / \mathrm{g}$ & \\
\hline $\mathrm{Pm}-144$ & $0.045+/-0.064$ & 0.11 & $\mathrm{pClig}$ & $u$ \\
\hline$P_{m-146}$ & $0.018+/-0.078$ & 0.14 & $\mathrm{pCi} / \mathrm{g}$ & $u$ \\
\hline Ru-106 & $0.24+1-0.56$ & 0.96 & $\mathrm{pCi} / \mathrm{g}$ & $u$ \\
\hline Sb-125 & $-0.12+/-0.15$ & 0.29 & $\mathrm{pCi} / \mathrm{g}$ & U \\
\hline Th-234 & $3.6+1-1.4$ & 1.9 & $\mathrm{pCi} / \mathrm{g}$ & $T$ \\
\hline TI-208 & $0.62+1-0.16$ & 0.14 & $\mathrm{pCi} / \mathrm{g}$ & \\
\hline U-235 & $-0.18+/-0.39$ & 0.70 & $\mathrm{pCi} / \mathrm{g}$ & $U$ \\
\hline$\overline{Y-88}$ & $0.006+/-0.081$ & 0.15 & $\mathrm{pCi} / \mathrm{g}$ & $U$ \\
\hline
\end{tabular}

Data Package ID: GSS0305155-1

Paragon Analytics Inc. 


\section{Gamma Spectroscopy Results}

\section{Method PAI 713R8}

\section{Sample Results}

Client Name: Bechtel Nevada

Client Project Name: CAU 523

Client Project Number: V1979
Page: 20 of 22

Reported on: Thursday, May 29, 2003 $10: 41: 46$

Laboratory Name: Paragon Analytics, inc.

PAI Work Order: 0305155
Field ID: $192231-1$

Lab ID: 0305155-10
Sample Matrix: Soil

Date Prepared: 28-May-03

Prep SOP: PAI 739R6

Prep Batch: GS02051
Date Collected: 20-May-03

Date Analyzed: 28-May-03

Analytical SOP: PAI 713R8

Spectrum Code: 030742D08A
Final Aliquot: $417.5 \mathrm{~g}$

Report Basis: Dry Weight

Count Time (min.): 30

Library: LNG_GAM-A-DO

\section{Target Nuclide}

Result +1- 2 s TPU

\section{MDC}

Reporting Units

\section{Lab Qualifier}

Abbreviations:

TPU - Total Propagated Uncertainty (see PAI SOP 743)

$U$ - Resutt is less than the sample specific MDC or less than the associated TPU.

MDC - Minimum Detectable Concentration (see PAI SOP 709)

$Y_{1}-$ Chemical Yield is in control at $100-110 \%$. Quantitative Yield is assumed.

Y2 - Chemical Yieid outside default lintils.

LT - Result is less than Requested MDC, greater than sample specific MDC.

$S Q$ - Spectral quality prevents accurate quantitation.

$\mathrm{SI}$ - Nuclide identification and/or quantitation is tentative.

$\mathrm{TI}$ - Nuclide identfication is tentative.

$R$ - Nuctide has exceeded 8 halfives. 


\section{Gamma Spectroscopy Results}

\section{Method PAI 713R8}

\section{Sample Results}

Client Name: Bechtel Nevada

Client Project Name: CAU 523

Client Project Number: V1979

Field ID: $192231-3$

Lab ID:0305155-11
Sample Matrix: Soil

Date Prepared: 28-May-03

Prep SOP: PAI $739 R 6$

Prep Batch: GS02051
Page: 21 of 22

Reported on: Thursday, May 29, 2003

10:41:46

Laboratory Name: Paragon Analytics, Inc.

PAI Work Order: 0305155

\begin{tabular}{|c|c|c|c|c|c|}
\hline & Target Nuclide & Result +1- 2 s TPU & MDC & $\begin{array}{l}\text { Reporting } \\
\text { Units }\end{array}$ & Lab Qualifier \\
\hline & $A C-228$ & $1.70+/-0.45$ & 0.69 & $\mathrm{pCi} / \mathrm{g}$ & \\
\hline & Am-241 & $-0.22+/-0.44$ & 0.78 & $\mathrm{pCi} / \mathrm{g}$ & U \\
\hline & $\mathrm{Bi}-214$ & $1.30+/-0.35$ & 0.32 & $\mathrm{pCi} / \mathrm{g}$ & \\
\hline & Cd-109 & $3.0+/-1.6$ & 2.3 & $\mathrm{pCi} / \mathrm{g}$ & SI \\
\hline & $\mathrm{Ce}-144$ & $0.29+1-0.42$ & 0.70 & $\mathrm{pCi} / \mathrm{g}$ & $\vec{U}$ \\
\hline & Co-60 & $0.03+/-0.11$ & 0.20 & $\mathrm{pCi} / \mathrm{g}$ & $U$ \\
\hline & Cs-134 & $-0.063+1-0.099$ & 0.19 & $\mathrm{pCi} / \mathrm{g}$ & $\mathrm{U}$ \\
\hline & Cs-137 & $0.24+/-0.15$ & 0.22 & $\mathrm{pCi} / \mathrm{g}$ & \\
\hline & Eu-152 & $0.04+/-0.46$ & 0.85 & $\mathrm{pCi} / \mathrm{g}$ & $u$ \\
\hline & Eu-154 & $0.43+/-0.51$ & 0.83 & $\mathrm{pCi} / \mathrm{g}$ & $U$ \\
\hline & Eu-155 & $0.28+/-0.23$ & 0.35 & $\mathrm{pCl} / \mathrm{g}$ & U \\
\hline & $\mathrm{K}-40$ & $27.1+1-5.4$ & 1.9 & $\mathrm{pCi} / \mathrm{g}$ & \\
\hline & $\mathrm{Pb}-212$ & $2.22+1-0.43$ & 0.22 & $\mathrm{pCi} / \mathrm{g}$ & \\
\hline \multirow{9}{*}{$\cdot$} & $\mathrm{Pb}-214$ & $1.32+/-0.31$ & 0.29 & $\mathrm{pCi} / \mathrm{g}$ & \\
\hline & $P m-144$ & $-0.017+/-0.082$ & 0.15 & $\mathrm{pCi} / \mathrm{g}$ & $U$ \\
\hline & $\mathrm{Pm}-146$ & $-0.042+/-0.088$ & 0.16 & $\mathrm{pCi} / \mathrm{g}$ & $U$ \\
\hline & Ru-106 & $0.28+/-0.66$ & 1.1 & $\mathrm{pCi} / \mathrm{g}$ & $U$ \\
\hline & Sb-125 & $0.13+/-0.16$ & 0.26 & $\mathrm{pCi} / \mathrm{g}$ & U \\
\hline & Th-234 & $2.8+/-1.8$ & 2.7 & $\mathrm{pCi} / \mathrm{g}$ & $\mathrm{TI}$ \\
\hline & TI-208 & $0.56+/-0.17$ & 0.17 & $\mathrm{pCi} / \mathrm{g}$ & \\
\hline & U-235 & $0.17+/-0.40$ & 0.67 & $\mathrm{pCi} / \mathrm{g}$ & U \\
\hline & $Y-88$ & $-0.038+1-0.092$ & 0.17 & $\mathrm{pCi} / \mathrm{g}$ & $\mathrm{U}$ \\
\hline
\end{tabular}

Date Collected: 20-May-03

Date Analyzed: 28-May-03

Analytical SOP: PAI 713R8

Spectrum Code: 030632D06A
Final Aliquot: $403.2 \mathrm{~g}$

Report Basis: Dry Weight Count Time (min.): 30

Library: LNG_GAM-A-00

Data Package ID: GSS0305155-1 


\section{Gamma Spectroscopy Results}

\section{Method PAI 713R8}

\section{Sample Results}

Client Name: Bechtel Nevada

Client Project Name: CAU 523

Client Project Number: V1979
Page: 22 of 22

Reported on: Thursday, May 29, 2003 10:41:46

Laboratory Name: Paragon Analytics, Inc.

PAl Work Order: 0305155
Field ID: 192231-3

Lab ID:0305155-11
Sample Matrix: Soil

Date Prepared: 28-May-03

Prep SOP: PAI 739R6

Prep Batch: GS02051
Date Collected: 20-May-03

Date Analyzed: 28-May-03

Analytical SOP: PAI 713R8

Spectrum Code: 030632D06A
Final Aliquot: $403.2 \mathrm{~g}$

Report Basis: Dry Weight

Count Time (min.): 30

Library: LNG_GAM-A-00

\begin{tabular}{|c|c|c|c|c|}
\hline Target Nuclide & Result $+/ .2$ s TPU & MDC & $\begin{array}{c}\text { Reporting } \\
\text { Units }\end{array}$ & Lab Qualifier \\
\hline
\end{tabular}

\section{Comments:}

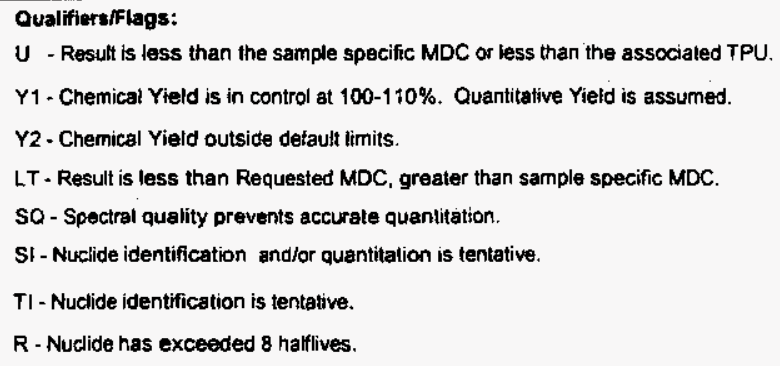

Abbreviations:

TPU - Total Propagated Uncertainty (see PAI SOP 743)

MDC - Minimum Detectable Concentration (see PAI SOP 709)

Data Package ID: GSS0305155-1 


\section{Gross Alpha/Beta Analysis by GFPC}

\section{PAl 724 Rev 8 \\ Sample Results}

Lab Name: Paragon Analytics, Inc.

Work Order Number: 0306110

Client Name: Bechtel Nevada

ClientProject ID: CAU 523 V1992

Field ID: 182224-1

Lab ID: 0306110-1
Sample Matrix: SOIL

Prep SOP: PAI 702 Rev 16

Date Collected: 20-May-03

Date Prepared: 24-Jun-03

Date Analyzed: 30-Jun-03
Prep Batch: AB030624-3

QCBatchiD: AB030624-3-2

Run ID: AB030624-3a

Count Time: 60 minutes
Final Aliquot: $0.500 \mathrm{~g}$

Result Units: $\mathrm{pCi} / \mathrm{g}$

File Name: abb0630b

Basis: As Received

\begin{tabular}{|c|c|c|c|c|}
\hline CASNO & Target Nuclide & Result +/- 2 s TPU & MDC & Lab Qualifier \\
\hline $14127-62-9$ & GROSS ALPHA & $9.1 \mathrm{E}-01+/-8.3 \mathrm{E}-01$ & $1.51 \mathrm{E}+00$ & $\mathrm{U}$ \\
\hline $12587-47-2$ & GROSS BETA & $3.3 \mathrm{E}+00+/-1.1 \mathrm{E}+00$ & $1.7 \mathrm{E}+00$ & $\mathrm{LT}$ \\
\hline
\end{tabular}

\section{Comments:}

\section{Quallfiers/Flags:}

$U$ - Result is less than the sample specific MDC.

Y1 - Chernical Yield is in control at 100-110\%. Quantitative Yield is assumed.

Y2 - Chernical Yield outside defautl limits.

LT - Result is less than Requesled MDC, greater than sample specific MDC.

\section{Abbreviations:}

TPU - Total Propagated Uncertainty (see PAI SOP 743)

MDC - Minimum Detectable Concentration (see PAI SOP 709)

M3 - The requested MDC was not met, but the reported activity is greater than the reported MDC.

$M$. The requested MDC was nol met.

Data Package ID: ABS0306110-1 


\section{Gross Alpha/Beta Analysis by GFPC}

\section{PAI 724 Rev 8}

\section{Sample Results}

Lab Name: Paragon Analytics, Inc.

Work Order Number: 0306110

Client Name: Bechtel Nevada

ClientProject ID: CAU 523 V1992

Fleld ID: 202227-1

Lab 1D: 0306110-2
Sample Matrix: SOHL

Prep SOP: PAI 702 Rev 16

Date Collected: 20-May-03

Date Prepared: 24-Jun-03

Date Analyzed: $30-J u n-03$
Prep Batch: AB030624-3

QCBatchID: AB030624-3-2

Run ID: AB030624-3a

Count Time: 60 minutes

Basis: Dry Weight
Final Aliquot: $0.502 \mathrm{~g}$

Result Units: pCi/g

File Name: abb0630b

\begin{tabular}{|c|c|c|c|c|}
\hline CASNO & Target Nuclide & Result +/- 2 s TPU & MDC & Lab Qualifier \\
\hline $14127-62-9$ & GROSS ALPHA & $7.2 \mathrm{E}+00+/-1.9 \mathrm{E}+00$ & $1.0 \mathrm{E}+00$ & \\
\hline $12587-47-2$ & GROSS BETA & $8.0 \mathrm{E}+00+/-1.8 \mathrm{E}+00$ & $1.8 \mathrm{E}+00$ & LT \\
\hline
\end{tabular}

\section{Comments:}

Qualiflers/Flags:
U - Result is less than the sample specific MDC.
Y1 - Chemical Yield is in control at 100-110\%. Quantitalive Yield is assumed.
Y2 - Chemical Yield outside default limits.
LT - Result is less than Requested MDC, greater than sample specific MDC.

Abbreviations:

TPU - Total Propagated Uncertainty (see PAI SOP 743)

MDC - Minimum Deteclable Concentrątion (see PAI SOP 709)

M3 - The requested MDC was not mel, but the reported activity is grealer than the reported MDC.

$M$ - The requested MDC was not met.

Data Package ID: ABS0306110-1

Date Printed: Monday, July 14, 2003 


\title{
Gross Alpha/Beta Analysis by GFPC
}

\author{
PAl 724 Rev 8 \\ Sample Results
}

Lab Name: Paragon Analytics, Inc.

Work Order Number: 0306110

Client Name: Bechtel Nevada

ClientProject ID: CAU 523 V 1992

Field 1D: 202227-2

Lab ID: $0306110-3$
Sample Matrix: SOIL

Prep SOP: PAl 702 Rev 16

Date Collected: 20-May-03

Date Prepared: 24-Jun-03

Date Analyzed: 30-Jun-03
Prep Batch: AB030624-3

QCBatchID: AB030624-3-2

Run ID: AB030624-3a

Count Time: 120 minutes

Basis: Dry Weight
Final Aliquot: $0.301 \mathrm{~g}$

Result Units: $\mathrm{pCi} / \mathrm{g}$

File Name: abb0630a

\begin{tabular}{|c|c|c|c|c|}
\hline CASNO & Target Nuclide & Result $+/-2$ s TPU & MDC & Lab Qualifier \\
\hline $14127-62-9$ & GROSS ALPHA & $8.6 \mathrm{E}+00+/-2.2 \mathrm{E}+00$ & $1.5 \mathrm{E}+00$ & \\
\hline $12587-47-2$ & GROSS BETA & $7.9 \mathrm{E}+00+/-1.8 \mathrm{E}+00$ & $2.0 \mathrm{E}+00$ & LT \\
\hline
\end{tabular}

\section{Comments:}

Quatifiers/Flags:

$U$ - Result is less than the sample specific MDC.

$Y 1$ - Chernical Yield is in controt at 100-110\%. Quantitative Yield is assumed.

Y2 - Cherrical Yieid outside default limits.

$L T$ - Result is less than Requested MDC. grealer than sample specific MDC.
Abbreviations:

TPU - Total Propagated Uncertainty (see PAI SOP 743)

MDC - Minimum Detectable Concentration (see PAI SOP 709)

\footnotetext{
$M 3$ - The requested MDC was not met, Dut the reported activity is greater than the reported MDC.

$M$ - The requesied MDC was not met.
}

Data Package ID: ABS0306110-1 


\section{Gross Alpha/Beta Analysis by GFPC}

PAl 724 Rev 8

Sample Results

Lab Name: Paragon Analytics, inc.

Work Order Number: 0306110

Client Name: Bechtel Nevada

ClientProject ID: CAU 523 V 1992

Field ID: 202227-3

Lab ID: $0306110-4$
Sample Matrix: SOIL

Prep SOP: PAI 702 Rev 16

Date Collected: 20-May-03

Date Prepared: 24-Jun-03

Date Analyzed: 30-Jun-03
Prep Batch: AB030624-3

QCBatchID: AB030624-3-2

Run ID: AB030624-3a

Count Time; 60 minutes

Basis: As Received
Final Aliquot: $0.403 \mathrm{~g}$

Result Units: $\mathrm{pCi} / \mathrm{g}$

Flle Name: abb0630b

\begin{tabular}{|c|c|c|c|c|}
\hline CASNO & Target Nuclide & Result +/- 2 s TPU & MDC & Lab Qualifier \\
\hline $14127-62-9$ & GROSS ALPHA & $3.3 E+00+/-1.5 E+00$ & $1.5 E+00$ & LT \\
\hline $12587-47-2$ & GROSS BETA & $3.4 E+00+/-1.3 E+00$ & $2.1 E+00$ & $L T$ \\
\hline
\end{tabular}

\section{Comments:}

\section{Qualifters/Flags:}

$U$ - Resull is less than the sample specific MDC.

Y1 - Chemical Yeld is in control at 100-110\%. Quantitative Yield is assumed.

Y2 - Chemical Yield outside defaull limits.

LT - Result is less than Requested MDC, greater than sample specific MDC.

\section{Abbreviations:}

TPU - Total Propagated Uncertainty (see PAI SOP 743)

MDC - Minimum Detectable Concentration (see PAI SOP 709)

M3 - The requested MDC was not met, but the reported activity is greater than the reported MOC

$M$ - The requested MOC was not met.

Data Package ID: ABS0306110-1 


\title{
Gross Alpha/Beta Analysis by GFPC
}

\author{
PAl 724 Rev 8
}

Sample Results

Lab Name: Paragon Analytics, Inc.

Work Order Number: 0306110

Client Name: Bechtel Nevada

ClientProject ID: CAU 523 V1992

\begin{tabular}{|l|}
\hline Field ID: $192231-1$ \\
Lab ID: $0306110-5$ \\
\hline
\end{tabular}

Sample Matrix: SOIL Prep SOP: PAI 702 Rev 16

Date Collected: 20-May-03

Date Prepared: 24-Jun-03

Date Analyzed: 30-Jun-03
Prep Batch: AB030624-3

QCBatchID: AB030624-3-2

Run ID: AB030624-3a

Count Time: 60 minutes

Basis: Dry Weight
Final Aliquot: $1.00 \mathrm{~g}$

Result Units: $\mathrm{pCi} / \mathrm{g}$

Flle Name: abb0630b

\begin{tabular}{|c|c|c|c|c|}
\hline CASNO & Target Nuclide & Result $+/-2$ s TPU & MDC & Lab Qualifier \\
\hline $14127-62-9$ & GROSS ALPHA & $5.3 E+00+/-1.3 E+00$ & $7.0 E-01$ & \\
\hline $12587-47-2$ & GROSS BETA & $7.0 E+00+/-1.4 E+00$ & $1.0 E+00$ & LT \\
\hline
\end{tabular}

\section{Comments:}

\section{Qualfilers/Flags:}

$U$ - Result is less than the sample specific MOC

Y1 - Cherrical Yieto is in control at 100- $\mathbf{1 0 \%}$. Quantitative Yield is assumed.

Y2 - Chemical Yield outside default limits.

LT - Result is less than Requested MDC. grealer than sample specific MDC.

\section{Abbreviations:}

TPU - Total Propagated Uncertainly (see PAI SOP 743)

MOC - Minimum Delectabie Concentration (see PAI SOP 709)

M3 - The requested MDC was not met, but the reported activity is greater than the reported MDC.

$M$ - The requested MDC was not met.

\section{Data Package ID: ABS0306110-1}




\title{
Gross Alpha/Beta Analysis by GFPC
}

\author{
PAI 724 Rev 8 \\ Sample Duplicate Results
}

Lab Name: Paragon Analytics, Inc.

Work Order Number: 0306110

Cllent Name: Bechtel Nevada

ClientProject ID: CAU 523 V1992

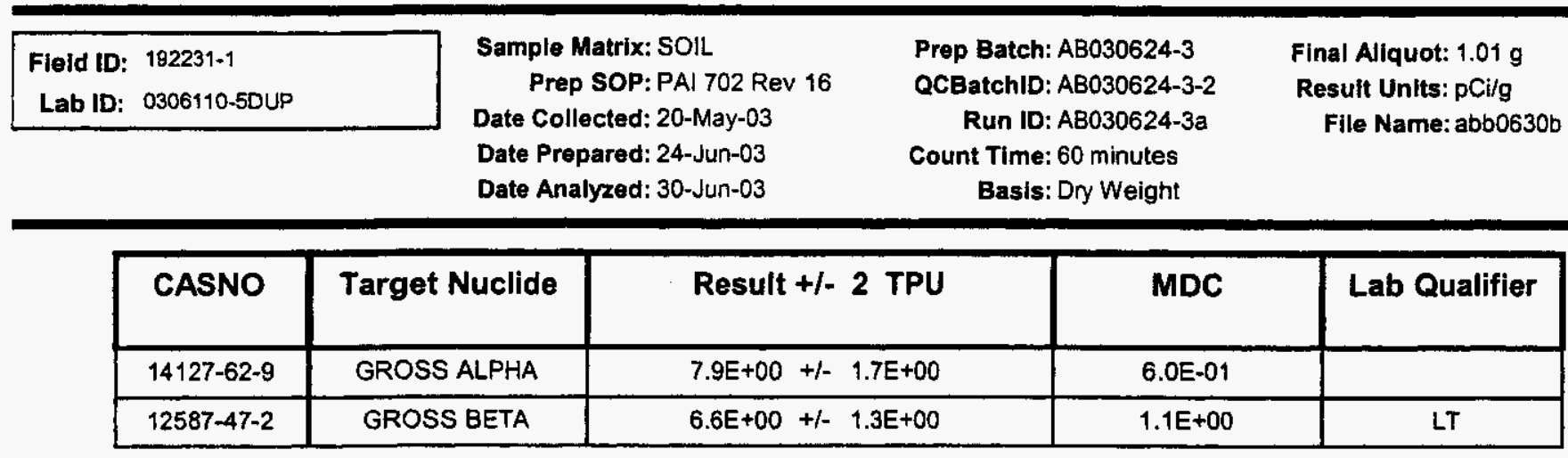

\section{Comments:}

\section{Qualifiera/Flags:}

$U$ - Result is less than the sample specific MDC.

$Y_{1}$ - Chemical Yield is in control at $100-110 \%$. Quantitative yield is assumed.

Y2 - Chemical Yield ouiside default timits.

LT - Resull is less than Requesled MDC, greater than sample specific MDC

$M$ - The requested MDC was not met.

M3 - The requested MDC was nol met, but thereported activily is greater than the reported MDC.

\section{Abbreviations:}

TPU - Tolai Propagated Uncertainty (see PAI SOP 743)

MDC - Mirimum Deteclabie Concentration (see PAI SOP 709)

Data Package ID: ABS0306110-1

Date Printed: Monday, July 14, 2003

Page 1 of 1 


\section{Gross Alpha/Beta Analysis by GFPC}

\section{PAl 724 Rev 8 \\ Sample Results}

Lab Name: Paragon Analytics, Inc.

Work Order Number: 0306110

Client Name: Bechtel Nevada

ClientProject ID: CAU 523 V 1992

Field ID: $192231-3$

Lab ID: $0306110-6$
Sample Matrix: SOIL

Prep SOP: PAl 702 Rev 16

Date Collected: 20-May-03

Date Prepared: 24-Jun-03

Date Analyzed: $30-\mathrm{Jun}-03$
Prep Batch: AB030624-3

QCBatchID: AB030624-3-2

Run ID: AB030624-3a

Count Time: 60 minutes

Basis: Dry Weight
Final Aliquot: $1.00 \mathrm{~g}$

Result Units: $p C i / g$

Flle Name: abb0630b

\begin{tabular}{|c|c|c|c|c|}
\hline CASNO & Target Nuclide & Result $+/-2$ s TPU & MDC & Lab Qualifier \\
\hline $14127-62-9$ & GROSS ALPHA & $6.1 \mathrm{E}+00+/-1.4 \mathrm{E}+00$ & $6.0 E-01$ & \\
\hline $12587-47-2$ & GROSS BETA & $8.1 \mathrm{E}+00+\% 1.6 \mathrm{E}+00$ & $1.0 \mathrm{E}+00$ & LT \\
\hline
\end{tabular}

\section{Comments:}

\section{Qualfiers/Flags:}

$U$ - Result is less than the sartple specific MDC.

Y1 - Chemical Yield is in control at 100-110\%. Quantitative Yield is assumed.

Y2 - Chemical Yield outside default limits.

LT - Result is less than Requested MDC, greater than sample specific MDC
Abbreviations:

TPU - Total Propagaled Uncertainty (see PAI SOP 743)

MOC - Minimum Detectable Concentration (see PAI SOP 709)

M3 - The requested MDC was not met, but the reported activity is greater than the reported MDC.

$M$ - The requested MDC was not met.

Data Package ID: ABS0306110-1

Date Printed: Monday, July 14, 2003 
Date: November 2003

\section{THIS PAGE INTENTIONALLY LEFT BLANK}


Closure Report - CAU 523

Section: Appendix A

Revision: 0

Date: November 2003

\section{SAMPLE DELIVERY GROUP}

\section{V2004}




\section{THIS PAGE INTENTIONALLY LEFT BLANK}




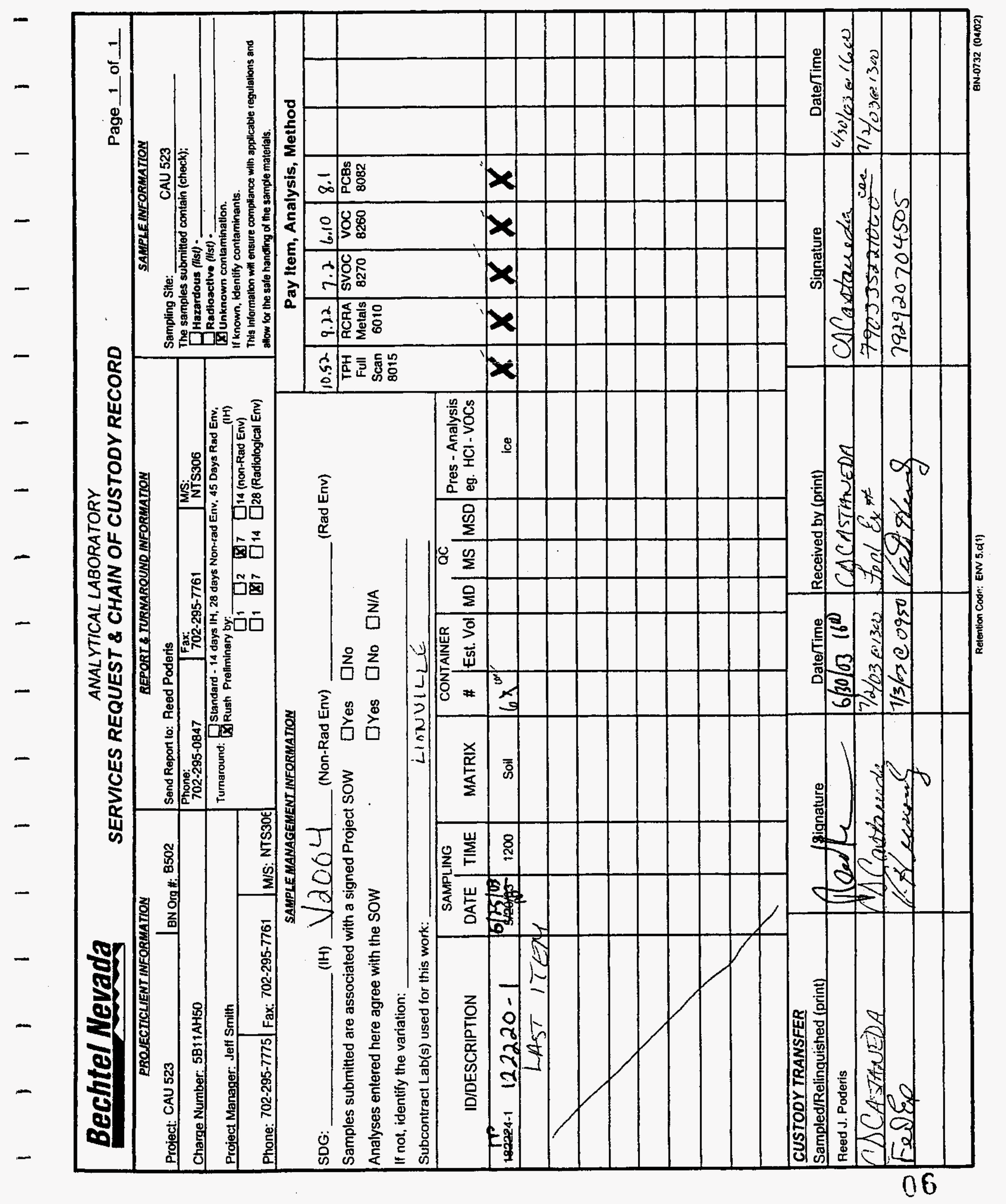


Lionville Laboratory, Inc.

INORGANICS DATA SUMMARY REPORT 07/10/03

LIEIT: BECHTEL NEVADA V2004

MORI: ORDER: 60052-001-001-0001-00
LVL LOT \#: $0307 L 738$

\begin{tabular}{|c|c|c|c|}
\hline RESULT & UNITS & $\begin{array}{l}\text { REPORTING } \\
\text { LIMIT }\end{array}$ & $\begin{array}{l}\text { DILUTION } \\
\text { FACTOR }\end{array}$ \\
\hline $\ln 2 x=x=0$ & $= \pm=x=x$ & 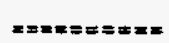 & 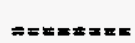 \\
\hline $0.12 \mathrm{u}$ & MG/KG & 0.12 & 1.0 \\
\hline 1.8 & MG/KG & 0.33 & 1.0 \\
\hline 78.6 & WG/KG & 0.02 & 1.0 \\
\hline 0.05 & MG/KG & 0.04 & 1.0 \\
\hline 3.3 & MG/KG & 0.10 & 1.0 \\
\hline $0.02 \mathrm{u}$ & MG/KG & 0.02 & 1.0 \\
\hline 17.2 & $M G / K G$ & 0.23 & 1.0 \\
\hline $0.42 u$ & MG/KG & 0.42 & 1.0 \\
\hline
\end{tabular}




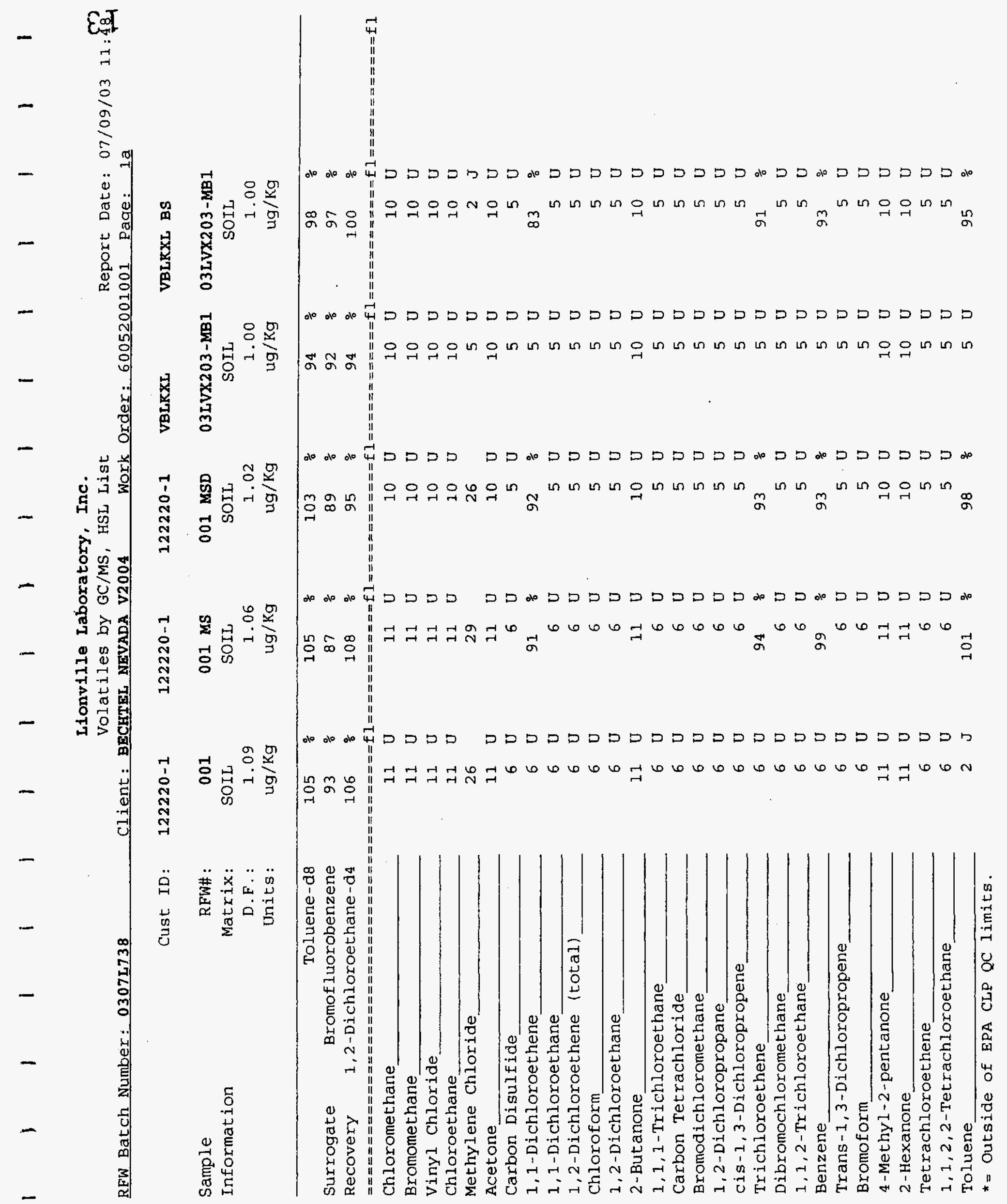




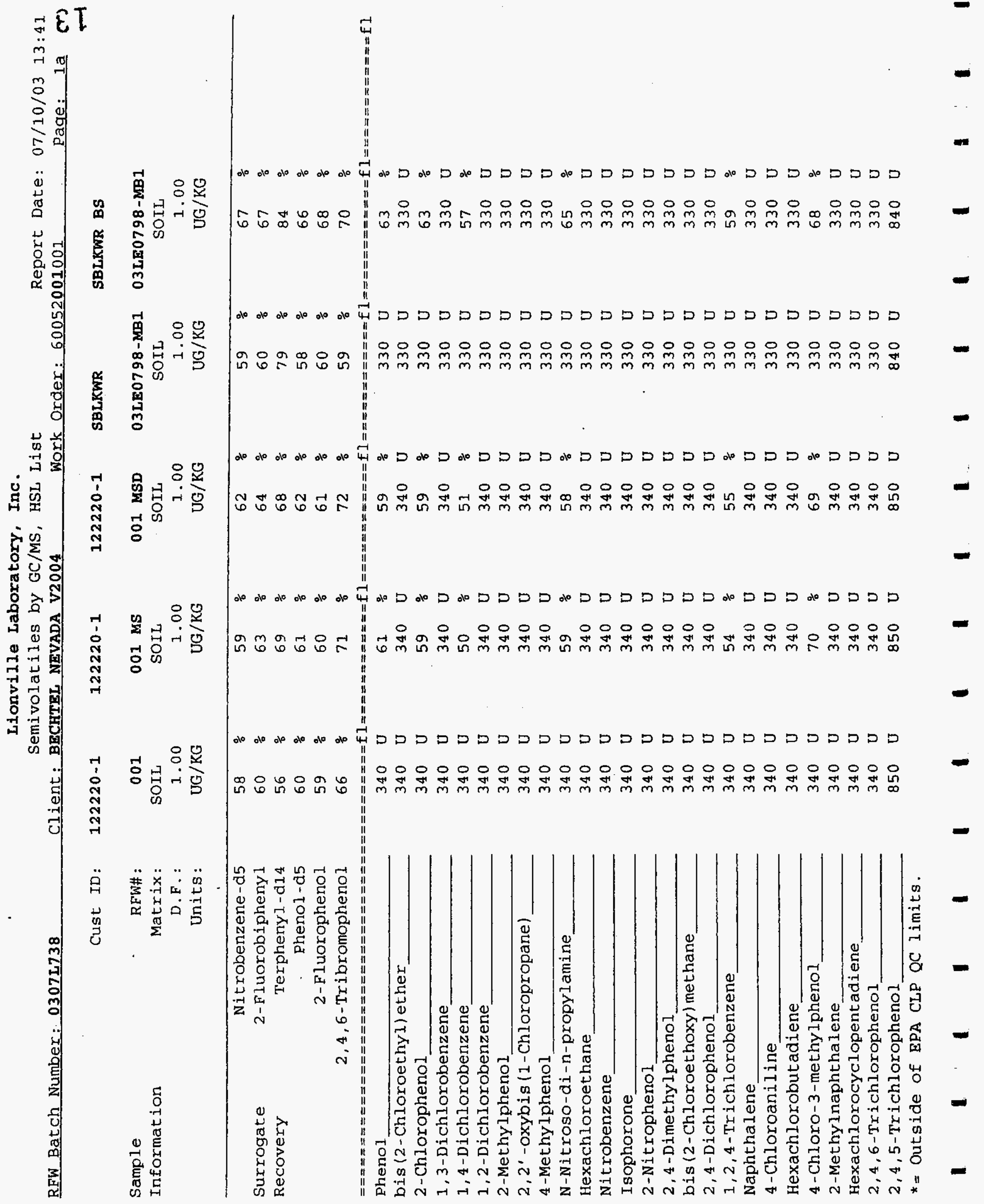




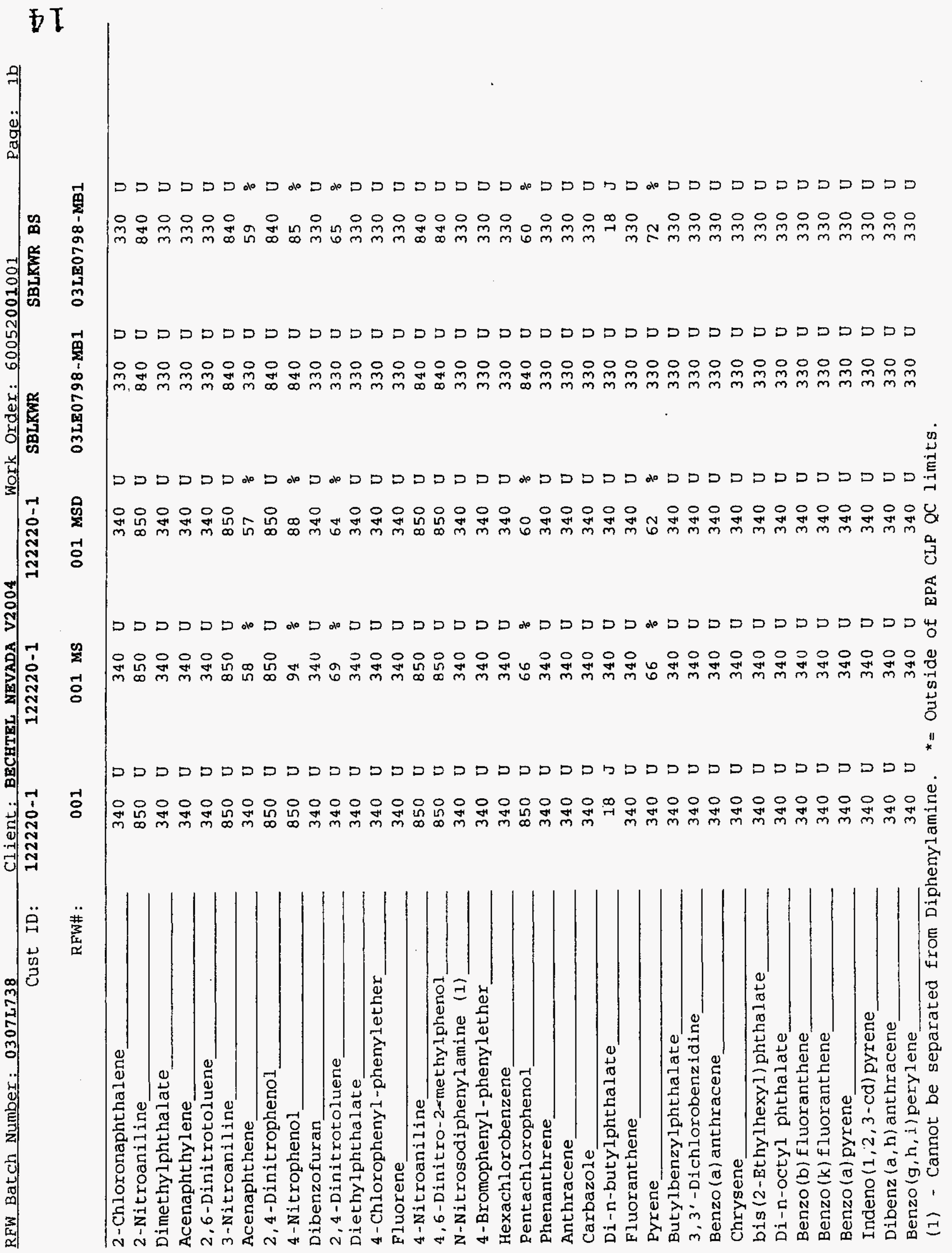




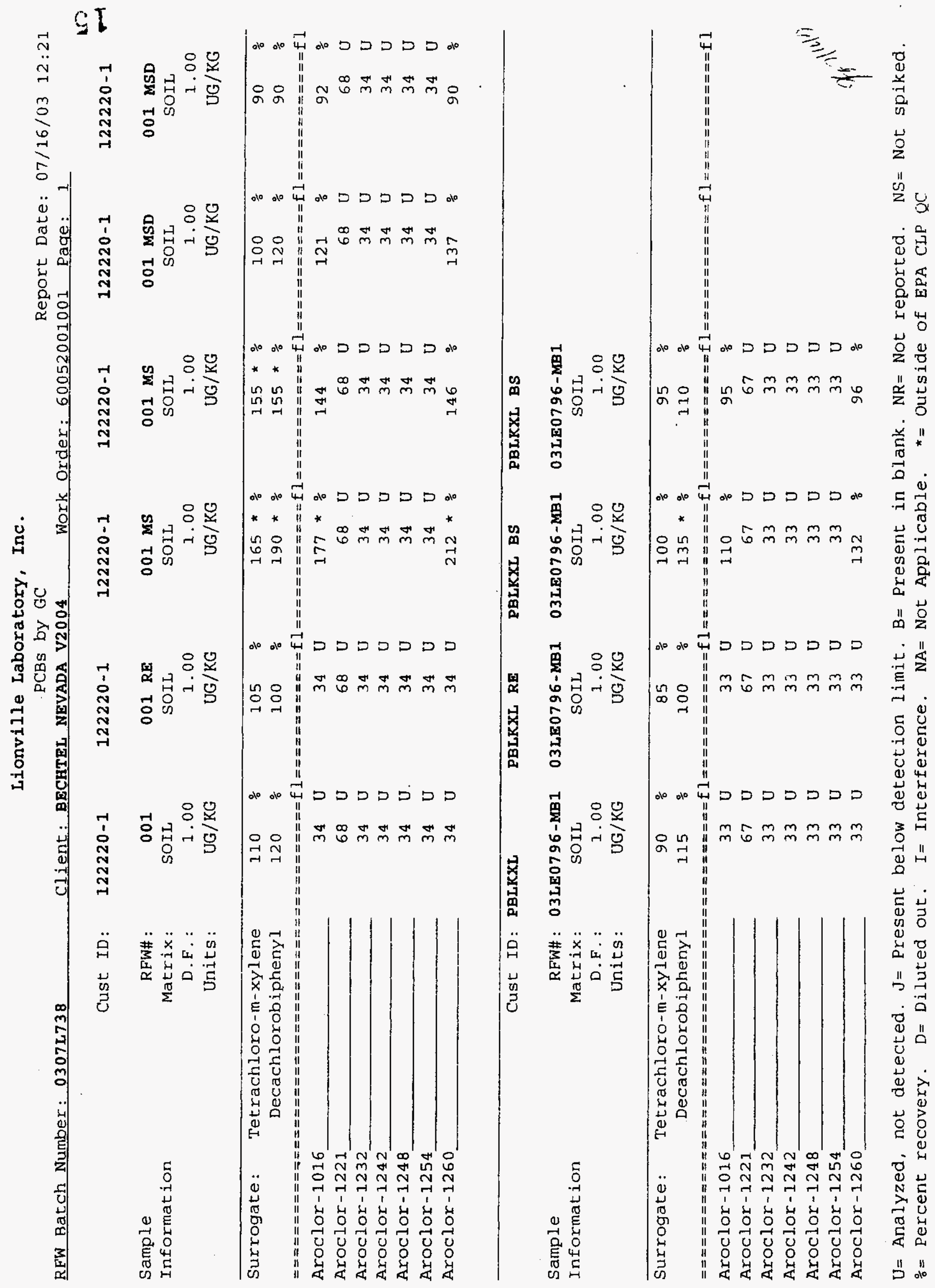




\section{T}
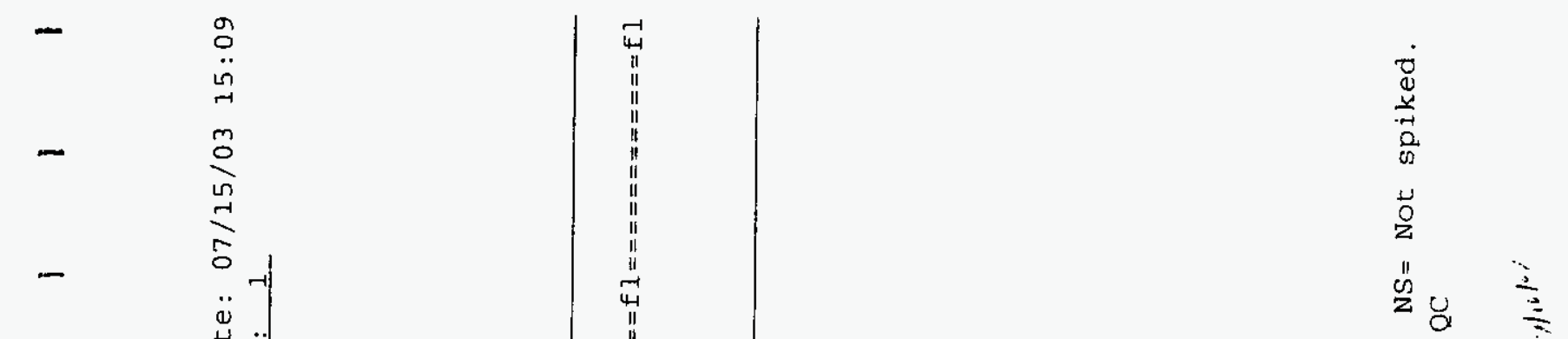

क्ष है

4

迥

4

苍 ญ

垈骂

节 *

동

- $\frac{1}{\pi}$

苨完

के

क

藏范

近苔

E

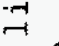

58

年

艺幽

(1) 4

包袋

3

吕

莸苛

क व

II

.

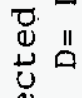

()

ชั

잉

.

क्र

늘

덩

엉

"11 


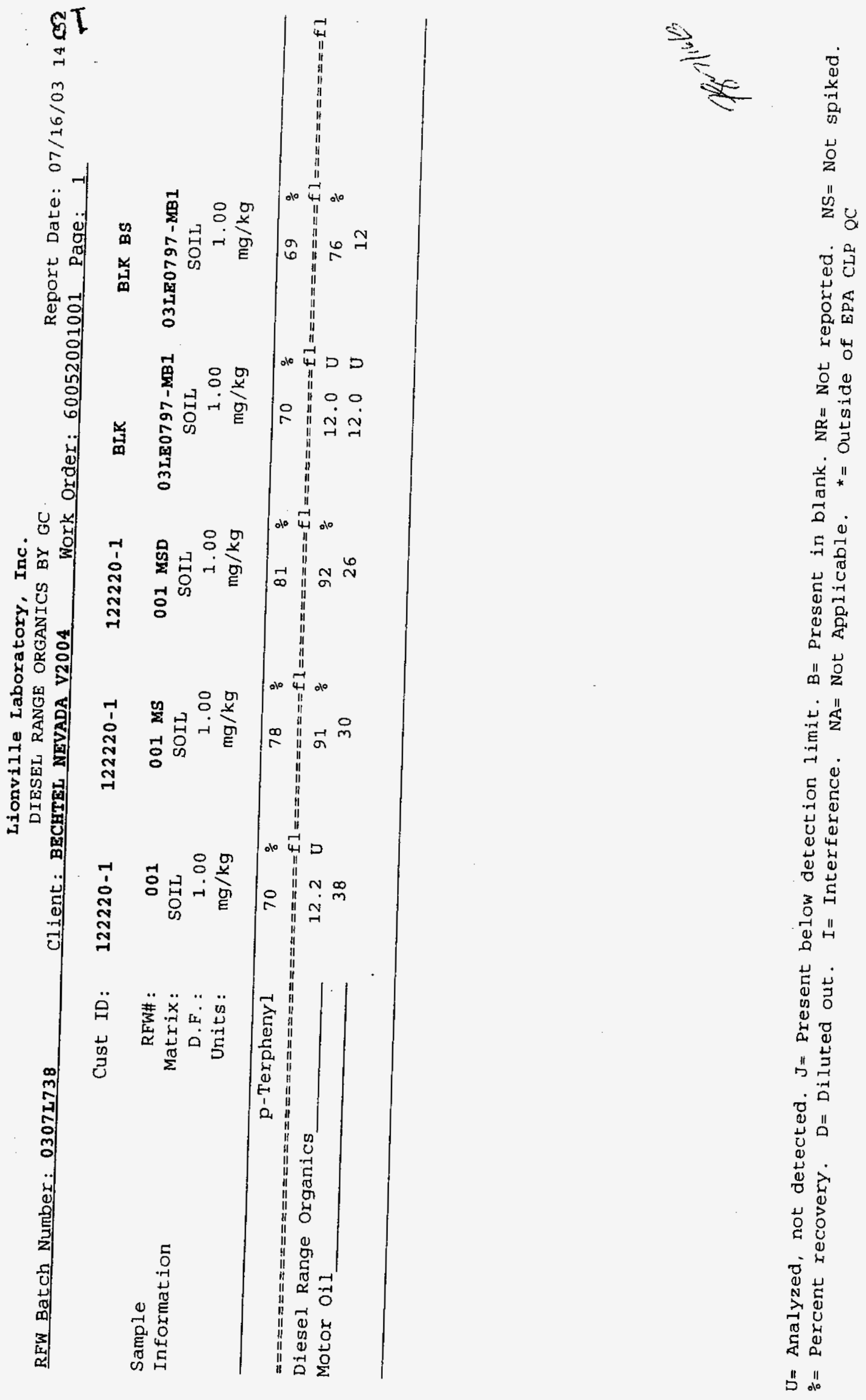


Closure Report - CAU 523

Section: Appendix A

Revision: 0

Date: November 2003

\section{SAMPLE DELIVERY GROUP}

V2029 


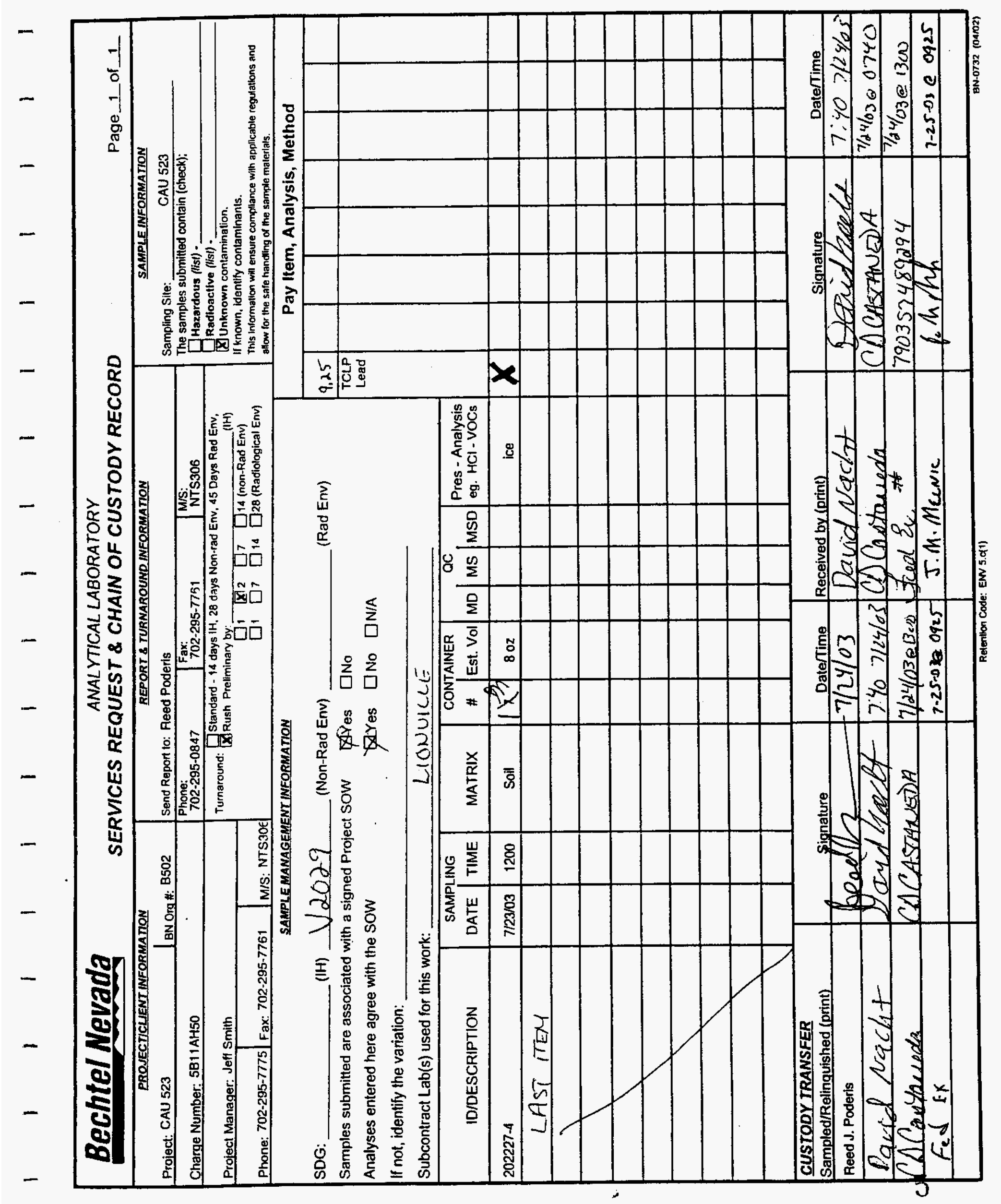


Lionville Laboratory, Inc.

INORGANICS DATA SUMMARY REPORT 07/29/03

CLIENT: BECHTEL NEVADA V2029

LVL LOT \#: 03071.937

WORK ORDER: 60052-001-001-0001-00

\begin{tabular}{|c|c|c|}
\hline SAMPLE & SITE ID & ANALYTE \\
\hline
\end{tabular}

\begin{tabular}{|c|c|c|c|}
\hline & & REPORTING & DILUTION \\
\hline RESULT & UNITS & LIMIT & FACTOR \\
\hline$\Rightarrow==\approx=x=$ & $m x=x=x$ & 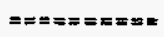 & $x=x=x=x=$ \\
\hline 38.5 & UG/L & 38.5 & 1 \\
\hline
\end{tabular}


Closure Report - CAU 523

Section: Appendix A

Revision: 0

Date: November 2003

\section{SAMPLE DELIVERY GROUP}

\section{V2038}


Date: November 2003

THIS PAGE INTENTIONALLY LEFT BLANK 


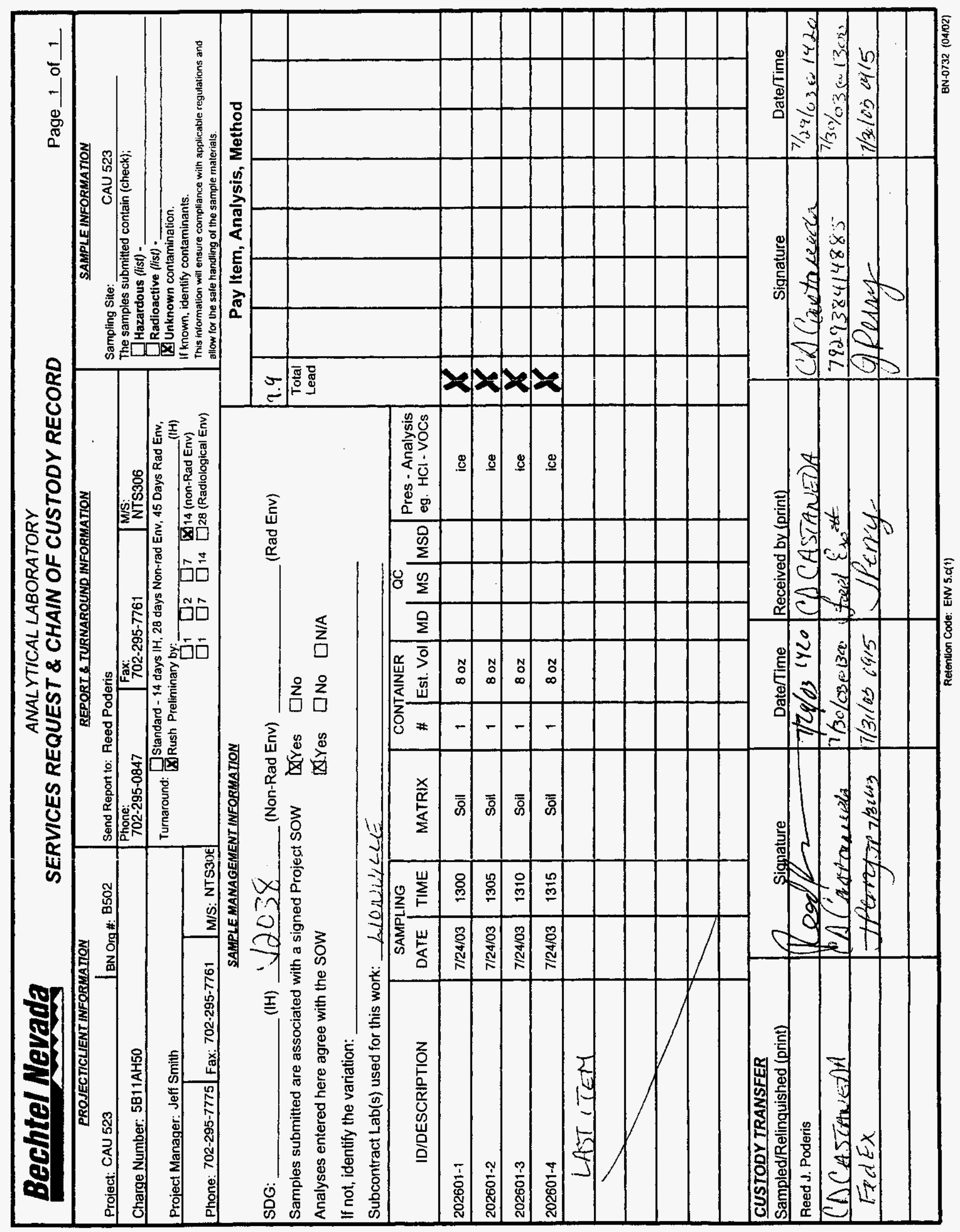


Lionville Laboratory, Inc.

INORGANICS DATA. SUMMARY REPORT 08/13/03

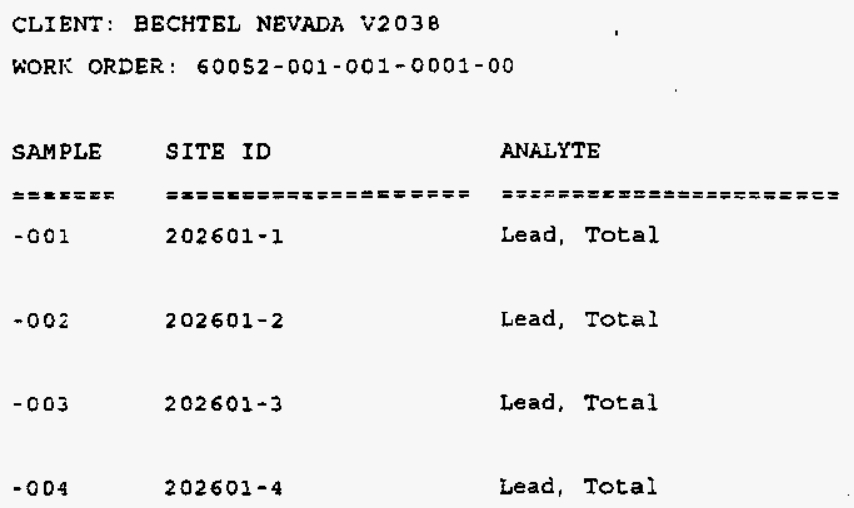

LVL LOT \#: $0307 \mathrm{L018}$

\begin{tabular}{|c|c|c|c|}
\hline & & REPORTING & DILUTION \\
\hline RESULT & UNITS & LIMIT & FACTOR \\
\hline$== \pm= \pm x=0$ & $x x=x==$ & $===\pi==x=m=$ & $x== \pm===\Rightarrow$ \\
\hline 6.6 & $M G / K G$ & 0.22 & 1.0 \\
\hline B. 6 & MG/KG & 0.23 & 1.0 \\
\hline 6.4 & MG/KG & 0.23 & 1.0 \\
\hline 7.2 & $M G / K G$ & 0.18 & 1.0 \\
\hline
\end{tabular}


Date: November 2003

\section{SAMPLE DELIVERY GROUP}

\section{V2040}




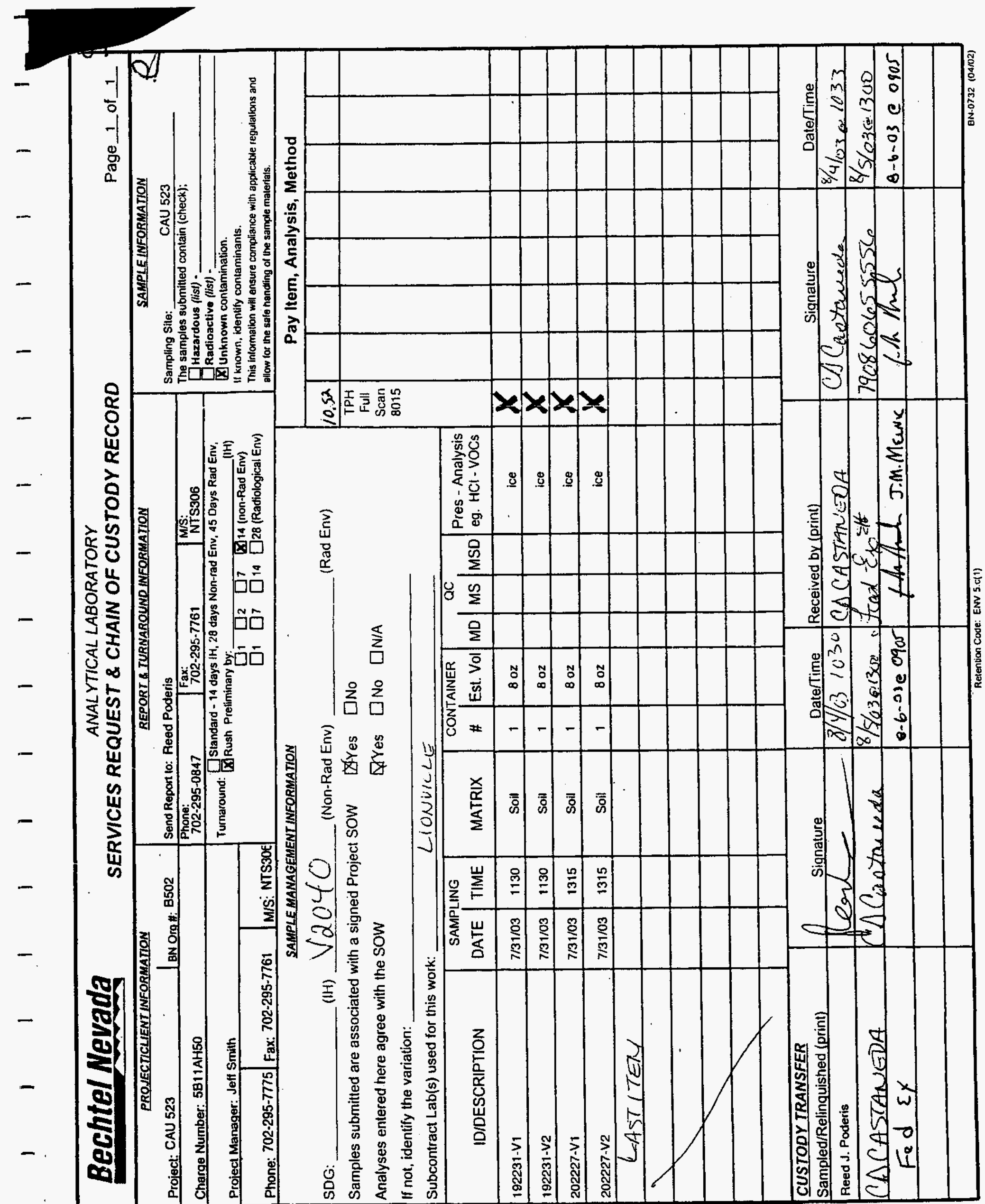


$E T$
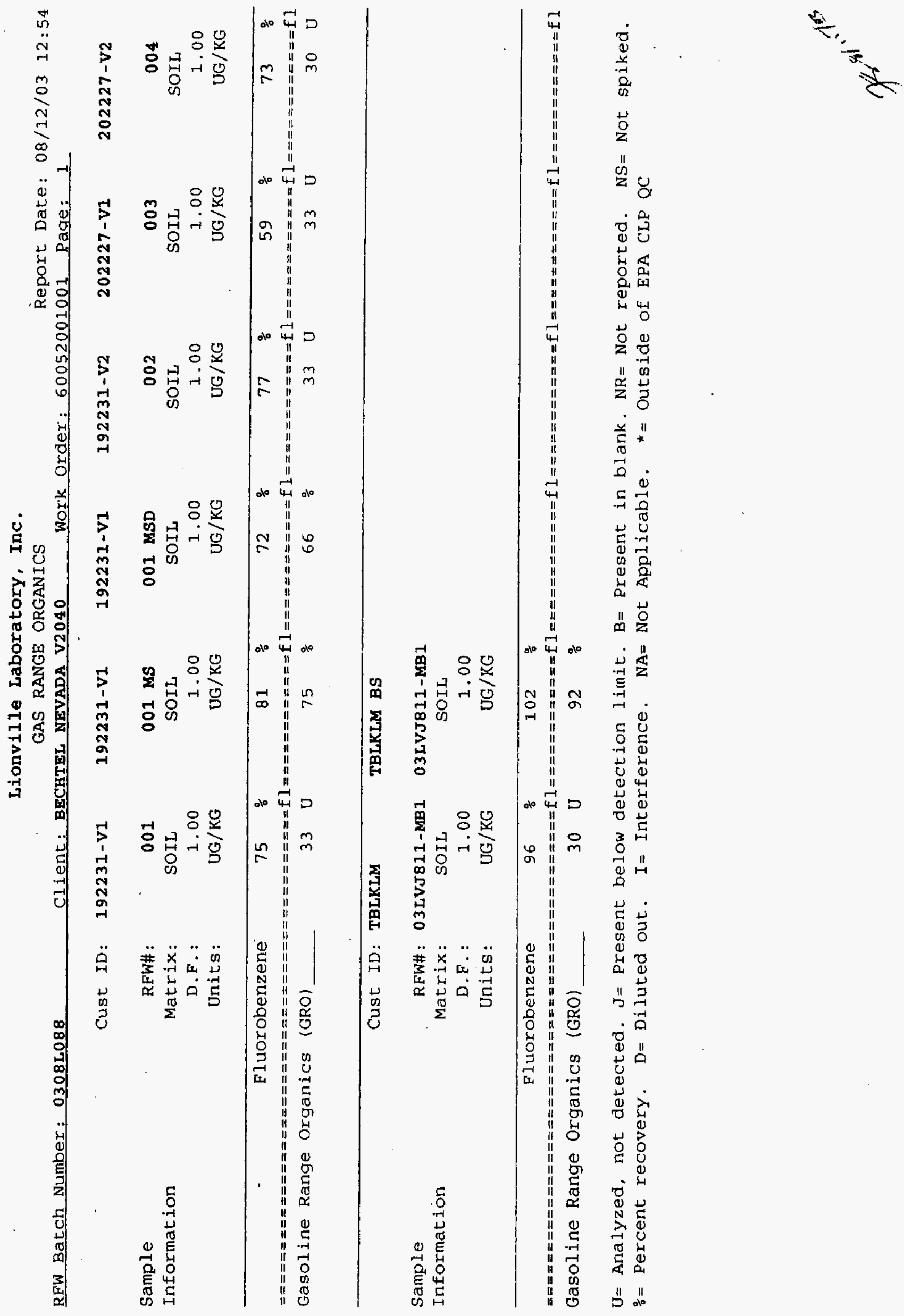
Date: November 2003

THIS PAGE INTENTIONALLY LEFT BLANK

$-$ 


\section{APPENDIX B}

\section{SECTORED HOUSEKEEPING SITE CLOSURE VERIFICATION FORMS}




\section{Sectored Housekeeping Site Closure Verification Form}

Closure Verification Date: 6/25/2003

CAU Number: 523

CAS Number: 04-22-04

CAS Description: Drums

Sector Designation: NTS Sector E

Housekeeping Site General Location: Nevada Test Site, U-4h

Elevation: 1,249 meters $(\mathrm{m})$

Northing: 4,107,156 m (UTM Zone 11) Easting: 584,045 m (UTM Zone 11)

Latitude: 37.10884

Longitude: -116.0541

Coordinate/Elevation Data Obtained from: North American Datum, 1927.

Site Access Route: Take Mercury Highway north to 4-04 Road. Cross 4-04 Road and continue north on Mercury Highway 0.9 mile to a dirt power line road. Turn right (east) on the power line road and proceed 0.8 mile. The site is on the north side of the road.

\begin{tabular}{|l|l|}
\hline \multicolumn{1}{|c|}{ Waste Item(s) Originally at Site } & \multicolumn{1}{c|}{ Apparent Waste Type* } \\
\hline Five Drums Inside the U-4h Crater & None, waste was previously removed. \\
\hline
\end{tabular}

* Ordinary, Scrap Metal, Asbestos, PCB, Salvageable, Hazardous, Radioactive, Mixed, Unknown, Other

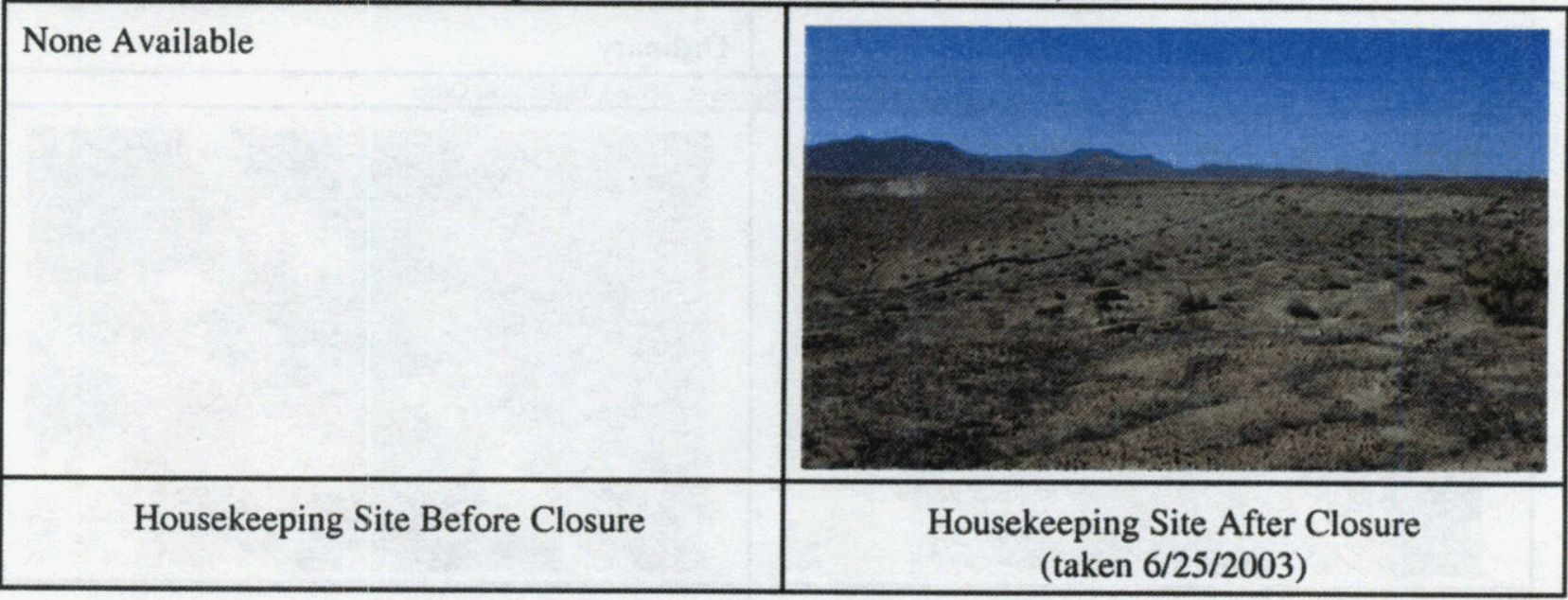

Current Site Description/Observations: No waste remained at this site during the closure activities. The site is currently clear of all waste material.

X No Further Action Required at Housekeeping Site

\begin{tabular}{lll} 
Reed J. Poderis, CEM & $11-6-03$ \\
\hline Corrective Action Coordinator/Designee & Signature & Date
\end{tabular}




\section{Sectored Housekeeping Site Closure Verification Form}

Closure Verification Date: 7/31/2003

CAU Number: 523

CAS Number: $12-22-20$

CAS Description: Drum

Sector Designation: NTS Sector F

Housekeeping Site General Location: Nevada Test Site, T-Tunnel Pond \#4

Elevation: 1,682 meters $(\mathrm{m})$

Northing: 4,119,103 m (UTM Zone 11) Easting: 574,387 m (UTM Zone 11)

Latitude: $37.21726 \quad$ Longitude: -116.16162

Coordinate/Elevation Data Obtained from: North American Datum, 1927.

Site Access Route: Take Mercury Highway north to Rainier Mesa Road. Turn left (northwest) on Rainier Mesa Road and proceed to 12-01 Road. Turn right (north) on 12-01 Road and follow the signs to the T-Tunnel portal. There is a locked barricade just as you reach the portal area. The I, J, and KTunnels are located on the ridge northeast of the barricade. Note: The site is located in a radioactive contamination area. Contact NNSAE/OCC at 5-4015 and Rad Safe for entry requirements.

\begin{tabular}{|l|l|}
\hline \multicolumn{1}{|c|}{ Waste Item(s) Originally at Site } & \multicolumn{1}{c|}{ Apparent Waste Type* } \\
\hline One empty 55-gallon antifreeze drum & Ordinary \\
\hline
\end{tabular}

* Ordinary, Scrap Metal, Asbestos, PCB, Salvageable, Hazardous, Radioactive, Mixed, Unknown, Other

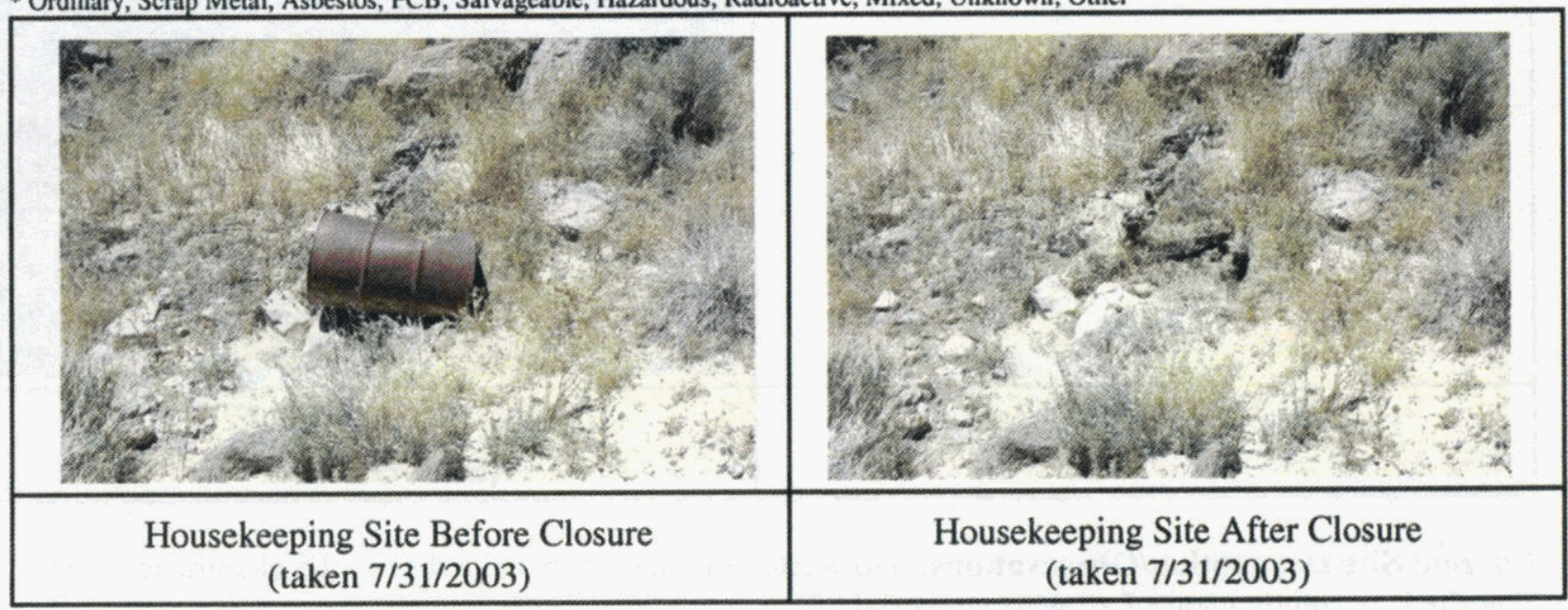

Current Site Description/Observations: The empty drum was disposed of in the Area 9 U-10c Landfill as ordinary waste. The site is currently clear of all waste material.

\section{No Further Action Required at Housekeeping Site}

$\begin{array}{ll}\mathrm{X} \text { No Further Action Required at Housekeeping Site } & 11-6.03 \\ \text { Reed J. Poderis, CEM } & \text { Signature }\end{array}$




\section{Sectored Housekeeping Site Closure Verification Form}

Closure Verification Date: 7/31/2003

CAU Number: 523

CAS Number: $16-22-29$

CAS Description: Drum

Sector Designation: NTS Sector D

Housekeeping Site General Location: Nevada Test Site, Area 16 Runway

Elevation: 1,652 meters $(\mathrm{m})$

Northing: 4,096,458 m (UTM Zone 11)

Latitude: 37.01335

Easting: $571,651 \mathrm{~m}$ (UTM Zone 11)

Longitude: -116.19455

Coordinate/Elevation Data Obtained from: North American Datum, 1927.

Site Access Route: Take Mercury Highway north to Pahute Mesa Road. Turn left (west) and continue to 16-02 Road. Turn left (south) on 16-02 Road and continue to the U-16a access road. Take the U-16a access road to the barricade. The site is $60 \mathrm{ft}$ east of the barricade, on the north side of the road.

\begin{tabular}{|c|l|}
\hline Waste Item(s) Originally at Site & \multicolumn{1}{|c|}{ Apparent Waste Type* } \\
\hline One empty 55-gallon drum. & None, waste was previously removed. \\
\hline
\end{tabular}

* Ordinary, Scrap Metal, Asbestos, PCB, Salvageable, Hazardous, Radioactive, Mixed, Unknown, Other

\begin{tabular}{|l|c|}
\hline None Available. & (taken 10/16/2003) \\
\hline Housekeeping Site Before Closure & $\begin{array}{c}\text { Housekeeping Site After Closure } \\
\text { (taks }\end{array}$ \\
\hline
\end{tabular}

Current Site Description/Observations: No waste remained at this site during the closure activities.

The site is currently clear of all waste material.

X No Further Action Required at Housekeeping Site

Reed J. Poderis, CEM

leed $\quad 11-6-03$

Corrective Action Coordinator/Designee

Signature

Date 


\section{Sectored Housekeeping Site Closure Verification Form}

Closure Verification Date: 7/24/2003

CAU Number: 523

CAS Number: $18-22-24$

CAS Description: Bottles - Lithium Bromide

Sector Designation: NTS Sector G

Housekeeping Site General Location: Nevada Test Site, Area 18 Water Sump Shack

Elevation: 1,719 meters $(\mathrm{m})$

Northing: 4,113,349 m (UTM Zone 11) Easting: 551,070 m (UTM Zone 11)

Latitude: 37.167

Longitude: -116.42478

Coordinate/Elevation Data Obtained from: North American Datum, 1927.

Site Access Route: Take Mercury Highway north to Tippipah Highway. Turn left (west) on Tippipah Highway and proceed to Pahute Mesa Road. Turn left (west) on Pahute Mesa Road and continue to Airport Road. Turn left (west) on Airport Road and proceed to Buckboard Mesa Road. Follow Buckboard Mesa Road north to RadSafe Marker (RSM) 104. The site is located $210 \mathrm{ft}$ south of the guard rail.

\begin{tabular}{|l|l|}
\hline \multicolumn{1}{|c|}{ Waste Item(s) Originally at Site } & \multicolumn{1}{c|}{ Apparent Waste Type* } \\
\hline $\begin{array}{l}\text { Lithium Bromide Bottles (not present). One soil- } \\
\text { filled 3-gallon bucket. }\end{array}$ & Ordinary \\
\hline
\end{tabular}

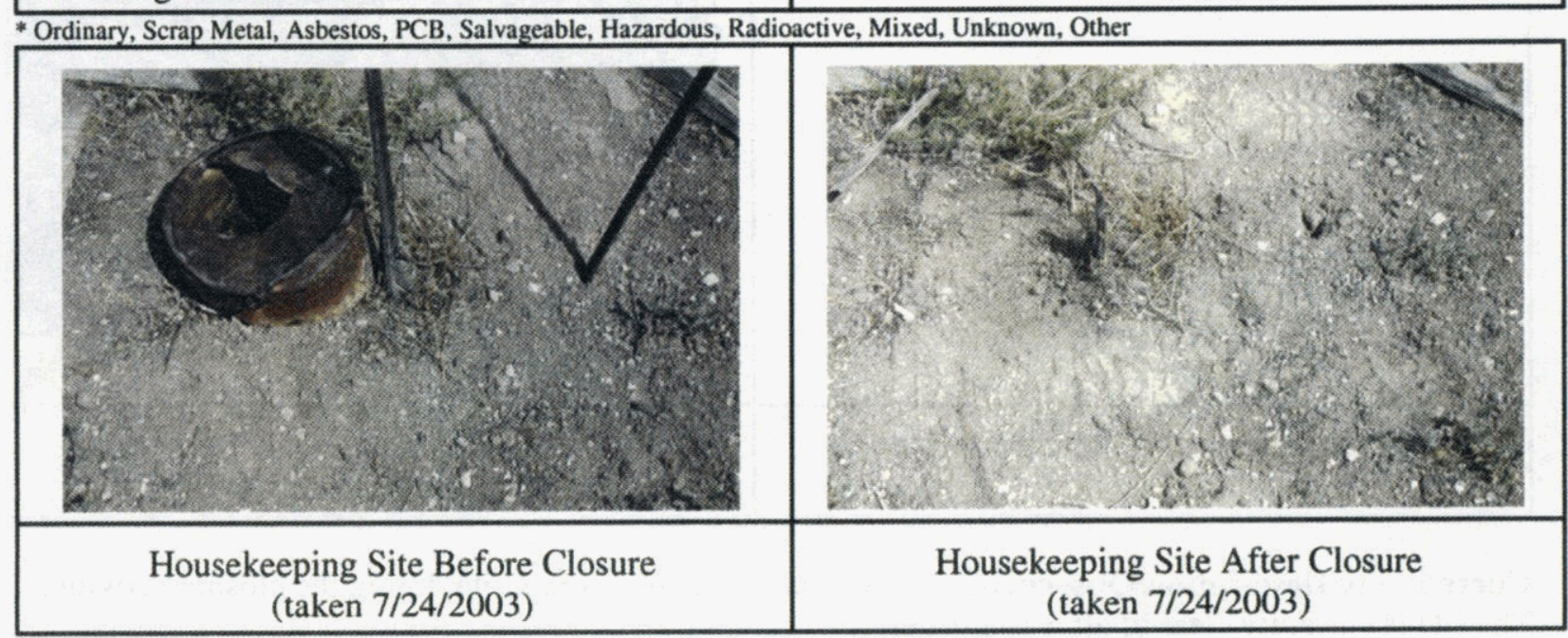

Current Site Description/Observations: After characterization sampling, the empty bucket was disposed of in the Area $9 \mathrm{U}-10 \mathrm{c}$ Landfill as ordinary waste.

$\mathrm{X}$ No Further Action Required at Housekeeping Site

Corrective Action Coordinator/Designee

Signature

$11-6-03$

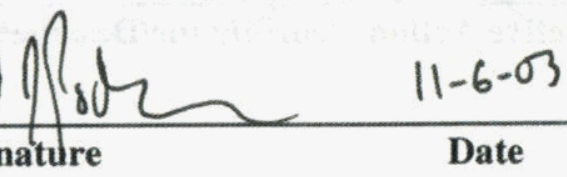




\section{Sectored Housekeeping Site Closure Verification Form}

Closure Verification Date: 7/24/2003

CAU Number: 523

CAS Number: 19-22-02

CAS Description: Drums (3)

Sector Designation: NTS Sector G

Housekeeping Site General Location: Nevada Test Site, U-19ar

Elevation: 2,042 meters (m)

Northing: 4,125,714 m (UTM Zone 11) Easting: 556,944 m (UTM Zone 11)

Latitude: $37.27811 \quad$ Longitude: -116.35768

Coordinate/Elevation Data Obtained from: North American Datum, 1927.

Site Access Route: Take Mercury Highway north to Tippipah Highway. Turn left (west) on Tippipah Highway and continue to Pahute Mesa Road. Turn left (west) on Pahute Mesa Road and proceed to Area 19. Travel to the semi-circle drive on the northwest side of Pahute Mesa Road and continue $600 \mathrm{ft}$ northwest to the U-19ar access road on the southeast side of the road. The U-19ar Crater is west and below the graded area. The site is located $300 \mathrm{ft}$ southwest of ground zero.

\begin{tabular}{|l|l|}
\hline \multicolumn{1}{|c|}{ Waste Item(s) Originally at Site } & \multicolumn{1}{c|}{ Apparent Waste Type* } \\
\hline Three drums & None, waste was previously removed. \\
\hline
\end{tabular}

* Ordinary, Scrap Metal, Asbestos, PCB, Salvageable, Hazardous, Radioactive, Mixed, Unknown, Other

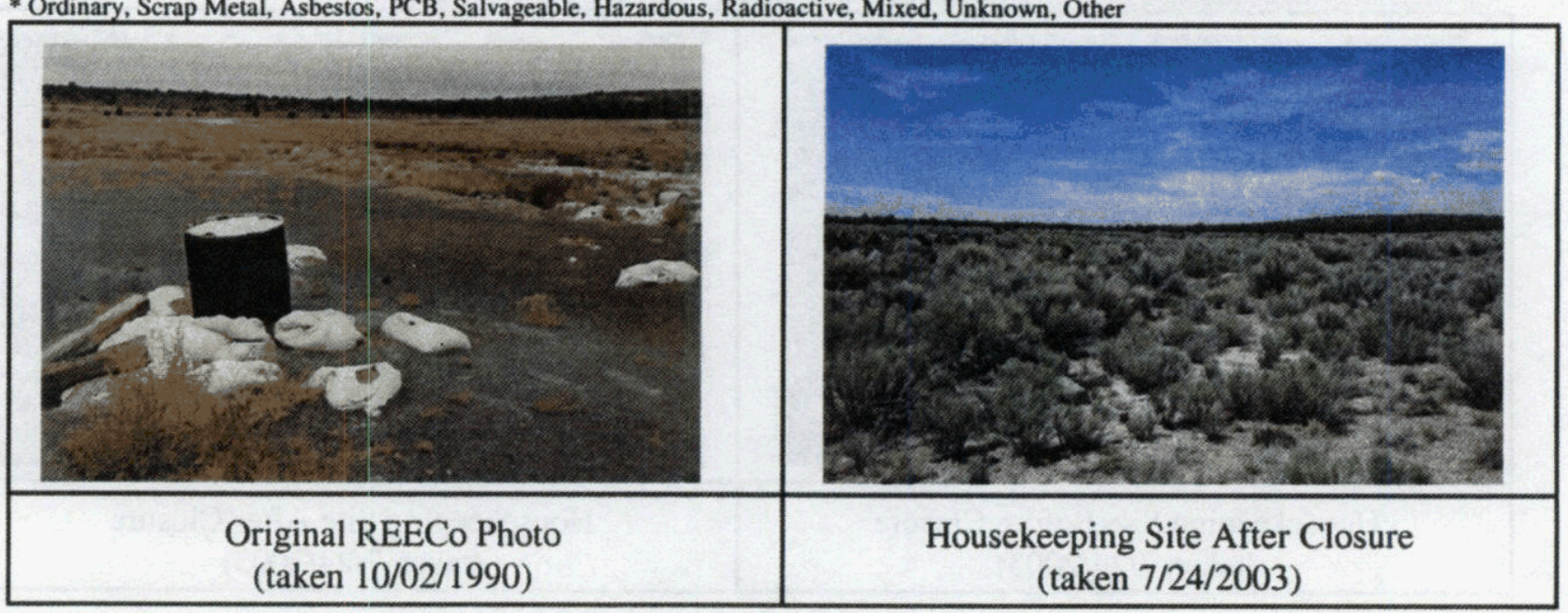

Current Site Description/Observations: No waste remained at this site during the Preliminary Assessment (PA) or the Bechtel Nevada (BN) closure activities. The site is currently clear of all waste material.

X No Further Action Required at Housekeeping Site

Reed J. Poderis, CEM

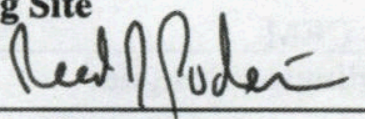

Signature
$11.6-03$

Date 


\section{Sectored Housekeeping Site Closure Verification Form}

Closure Verification Date: 7/24/2003

CAU Number: 523

CAS Number: 19-22-04

CAS Description: Drums (3)

Sector Designation: NTS Sector G

Housekeeping Site General Location: Nevada Test Site, U-19ay

Elevation: 2,048 meters (m)

Northing: 4,125,845 m (UTM Zone 11) Easting: 557,365 m (UTM Zone 11)

Latitude: $37.27926667 \quad$ Longitude: -116.3529167

Coordinate/Elevation Data Obtained from: North American Datum, 1927.

Site Access Route: Take Mercury Highway north to Pahute Mesa Road. Turn left (west) on Pahute Mesa Road and proceed to Area 19. Turn left (south) on the U-19ay access road and continue 0.2 mile. Site marker "A" is located on the right side of the road. Site marker "B" is located $150 \mathrm{ft}$ further down the road. Site marker "C" is located $600 \mathrm{ft}$ north of U-19ay ground zero.

\begin{tabular}{|c|c|}
\hline Waste Item(s) Originally at Site & \multicolumn{1}{|c|}{ Apparent Waste Type* } \\
\hline Three drums, only several steel rods remained. & None, waste was previously removed. \\
\hline
\end{tabular}

* Ordinary, Scrap Metal, Asbestos, PCB, Salvageable, Hazardous, Radioactive, Mixed, Unknown, Other

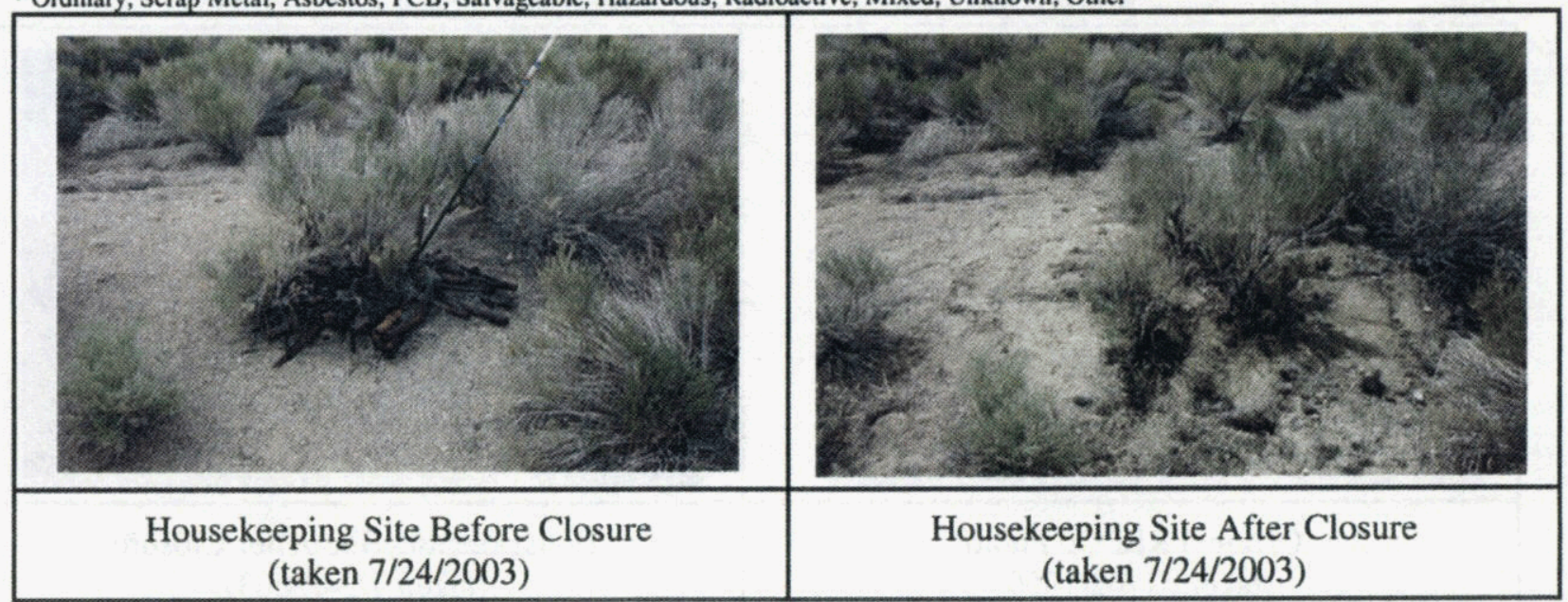

Current Site Description/Observations: The steel rods were disposed of in the Area 9 U-10c Landfill as ordinary waste. The site is currently clear of all waste material.

X No Further Action Required at Housekeeping Site

Reed J. Poderis, CEM

Corrective Action Coordinator/Designee

Signature
$11-603$

Date 


\section{Sectored Housekeeping Site Closure Verification Form}

Closure Verification Date: 7/31/2003

CAU Number: 523

CAS Number: 19-22-31

CAS Description: Can

Sector Designation: NTS Sector G

Housekeeping Site General Location: Nevada Test Site, Substation 19-10

Elevation: 2,066 meters $(\mathrm{m})$

Northing: 4,129,870 m (UTM Zone 11)

Latitude: 37.3153

Easting: $561,254 \mathrm{~m}$ (UTM Zone 11)

Longitude: -116.3087167

Coordinate/Elevation Data Obtained from: North American Datum, 1927.

Site Access Route: Take Mercury Highway north to Pahute Mesa Road. Turn left (west) on Pahute Mesa Road and proceed to Dead Horse Flats Road. Turn right (northeast) on Dead Horse Flats Road and proceed 0.4 mile past the U-19ac Crater to a dirt road on the left (west). Turn left and proceed 1.1 miles to RadSafe Marker (RSM) 19E4. Continue 2.0 miles to a power line road on the right. Turn right on the dirt road, travel up the hill less than $0.1 \mathrm{mi}$., and turn left on the power line road. The site is immediately on the left side of the road at the site marker.

\begin{tabular}{|l|l|}
\hline \multicolumn{1}{|c|}{ Waste Item(s) Originally at Site } & \multicolumn{1}{c|}{ Apparent Waste Type* } \\
\hline Several empty cans. & Ordinary. \\
\hline
\end{tabular}

* Ordinary, Scrap Metal, Asbestos, PCB, Salvageable, Hazardous, Radioactive, Mixed, Unknown, Other

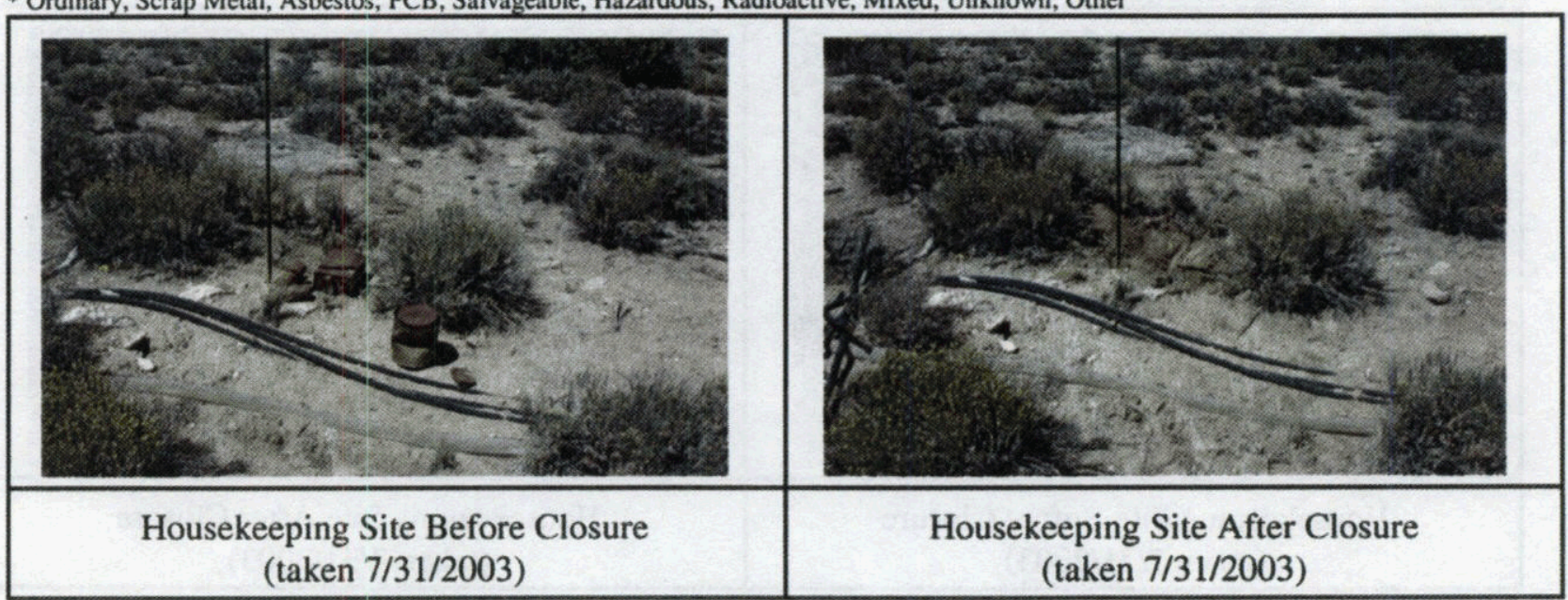

Current Site Description/Observations: The cans were disposed of in the Area 9 U-10c Landfill as ordinary waste. Due to their length and extent, the cables in the photos could not be removed. The site is currently clear of all waste material.

X No Further Action Required at Housekeeping Site

$\begin{array}{ccc}\text { Reed J. Poderis, CEM } & 11-603 \\ \text { Corrective Action Coordinator/Designee } & \text { Signature } & \text { Date }\end{array}$




\section{Sectored Housekeeping Site Closure Verification Form}

Closure Verification Date: 7/31/2003

CAU Number: 523

CAS Number: $19-22-32$

CAS Description: Can; Debris

Sector Designation: NTS Sector G

Housekeeping Site General Location: Nevada Test Site, U-19r

Elevation: 2,084 meters (m)

Northing: 4,123,738 m (UTM Zone 11) Easting: $561,857 \mathrm{~m}$ (UTM Zone 11)

Latitude: 37.25999

Longitude: -116.30243

Coordinate/Elevation Data Obtained from: North American Datum, 1927.

Site Access Route: Take Mercury Highway north to Pahute Mesa Road. Turn left (west) on Pahute Mesa Road and proceed to Dead Horse Flats Road. Turn right (northeast) on Dead Horse Flats Road and proceed 0.75 mile to a dirt road leading to the U-19r Crater on the right (east). Turn right on the dirt road and proceed 0.05 mile. The site marker is on the left (north) side of the dirt road.

\begin{tabular}{|l|l|}
\hline \multicolumn{1}{|c|}{ Waste Item(s) Originally at Site } & \multicolumn{1}{c|}{ Apparent Waste Type* } \\
\hline Empty can and debris & Ordinary. \\
\hline
\end{tabular}

* Ordinary, Scrap Metal, Asbestos, PCB, Salvageable, Hazardous, Radioactive, Mixed, Unknown, Other

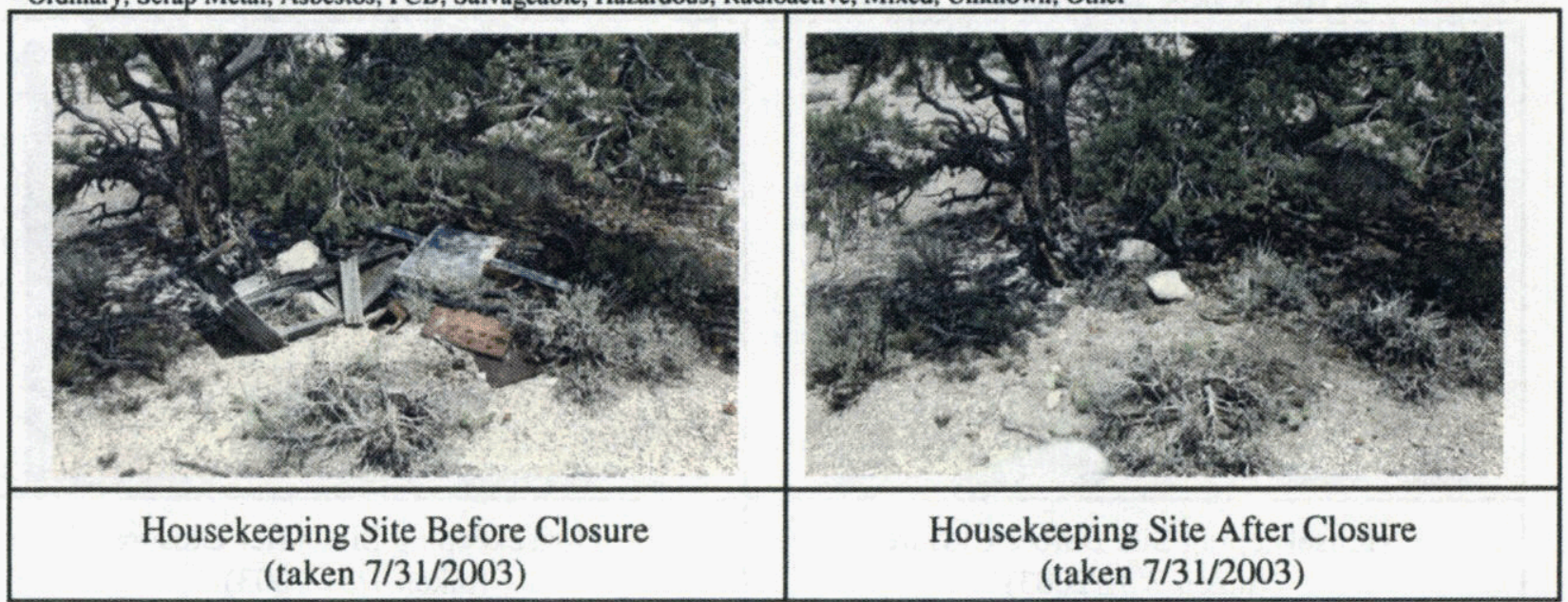

Current Site Description/Observations: The can and debris were disposed of in the Area 9 U-10c Landfill as ordinary waste. The site is currently clear of all waste material.

X No Further Action Required at Housekeeping Site

Reed J. Poderis, CEM

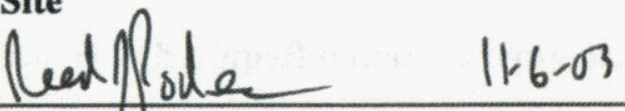

Corrective Action Coordinator/Designee

Signature

Date 


\section{Sectored Housekeeping Site Closure Verification Form}

Closure Verification Date: 7/24/2003

CAU Number: 523

CAS Number: 20-22-03

CAS Description: Drums

Sector Designation: NTS Sector G

Housekeeping Site General Location: Nevada Test Site, U-20p

Elevation: 1,693 meters $(\mathrm{m})$

Northing: 4,132,508 m (UTM Zone 11) Easting: 542,299 m (UTM Zone 11)

Latitude: 37.34013

Longitude: -116.52248

Coordinate/Elevation Data Obtained from: North American Datum, 1927.

Site Access Route: Take Mercury Highway north to Tippipah Highway. Turn left (west) on Tippipah Highway and proceed to Pahute Mesa Road. Turn left (west) on Pahute Mesa Road and proceed to Airport Road. Turn left (west) on Airport Road, which becomes Buckboard Mesa Road, and continue to Pahute Mesa Road. Turn left (northwest) on Pahute Mesa Road and travel to 20-03 Road. Turn right (north) on 20-03 Road and continue, staying to the left (west) at the fork, to the U-20p Crater at the end of the road.

\begin{tabular}{|l|l|}
\hline \multicolumn{1}{|c|}{ Waste Item(s) Originally at Site } & \multicolumn{1}{c|}{ Apparent Waste Type* } \\
\hline Several drums & None, drums were previously removed. \\
\hline
\end{tabular}

* Ordinary, Scrap Metal, Asbestos, PCB, Salvageable, Hazardous, Radioactive, Mixed, Unknown, Other

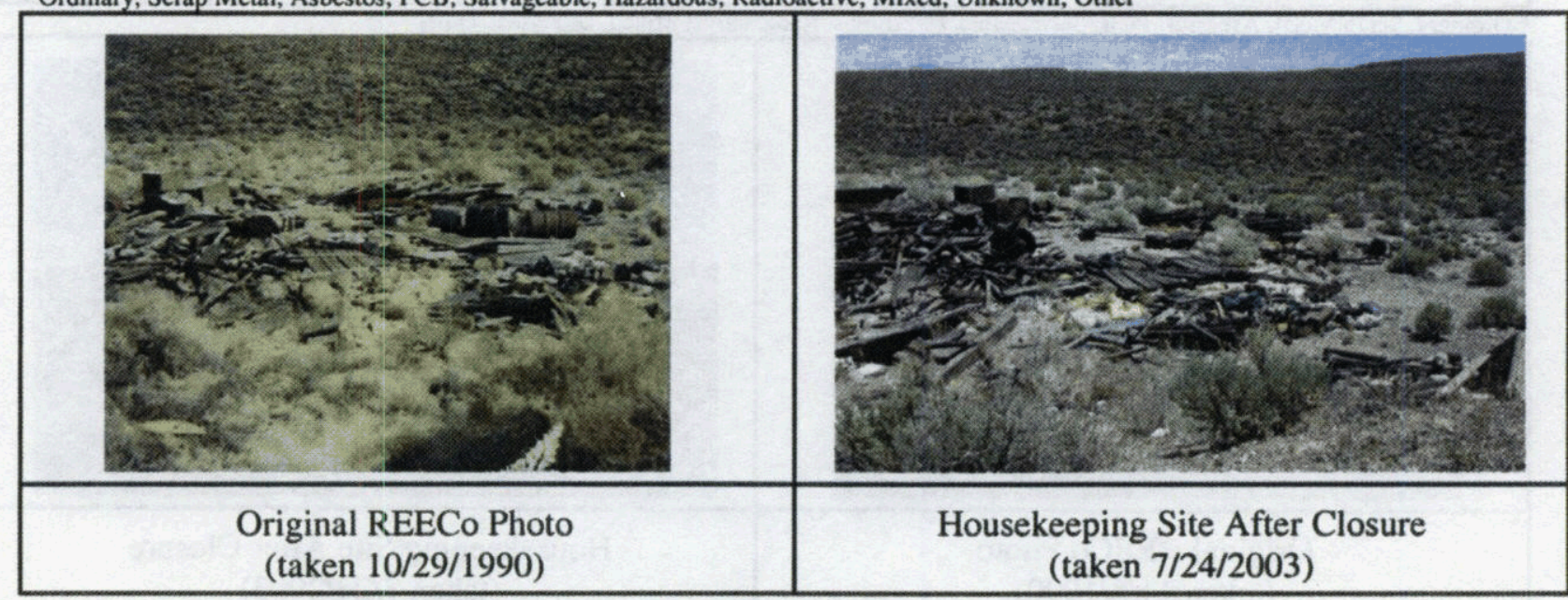

Current Site Description/Observations: No drums remained at this site during the Preliminary Assessment or the Bechtel Nevada closure activities. There is other debris present. However, the site is inaccessible by vehicle as the site is located within an potential crater area.

X No Further Action Required at Housekeeping Site

Reed J. Poderis, CEM

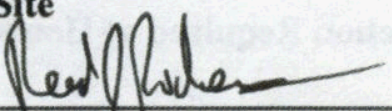

$116-03$

Corrective Action Coordinator/Designee

Signature

Date 


\section{Sectored Housekeeping Site Closure Verification Form}

Closure Verification Date: 7/24/2003

CAU Number: 523

CAS Number: 20-22-10

CAS Description: Drum

Sector Designation: NTS Sector G

Housekeeping Site General Location: Nevada Test Site, U-20ax

Elevation: 1,993 meters $(\mathrm{m})$

Northing: 4,121,725 m (UTM Zone 11) Easting: 550,438 m (UTM Zone 11)

Latitude: 37.24253

Longitude: -116.43133

Coordinate/Elevation Data Obtained from: North American Datum, 1927.

Site Access Route: Take Mercury Highway north to Tippipah Highway. Turn left (west) on Tippipah Highway and proceed to Pahute Mesa Road. Turn left (west) on Pahute Mesa Road and proceed to Airport Road. Turn left (west) on Airport Road, which becomes Buckboard Mesa Road, and proceed to Pahute Mesa Road. Turn right (northeast) on Pahute Mesa Road and travel 1.1 miles to a dirt road on the right (south). Turn right on the dirt road and continue past an old well and pond site. Proceed another $600 \mathrm{ft}$ to the site on the east side of the road.

\begin{tabular}{|l|l|}
\hline \multicolumn{1}{|c|}{ Waste Item(s) Originally at Site } & \multicolumn{1}{c|}{ Apparent Waste Type* } \\
\hline Several drums & None, drums were previously removed. \\
\hline
\end{tabular}

* Ordinary, Scrap Metal, Asbestos, PCB, Salvageable, Hazardous, Radioactive, Mixed, Unknown, Other

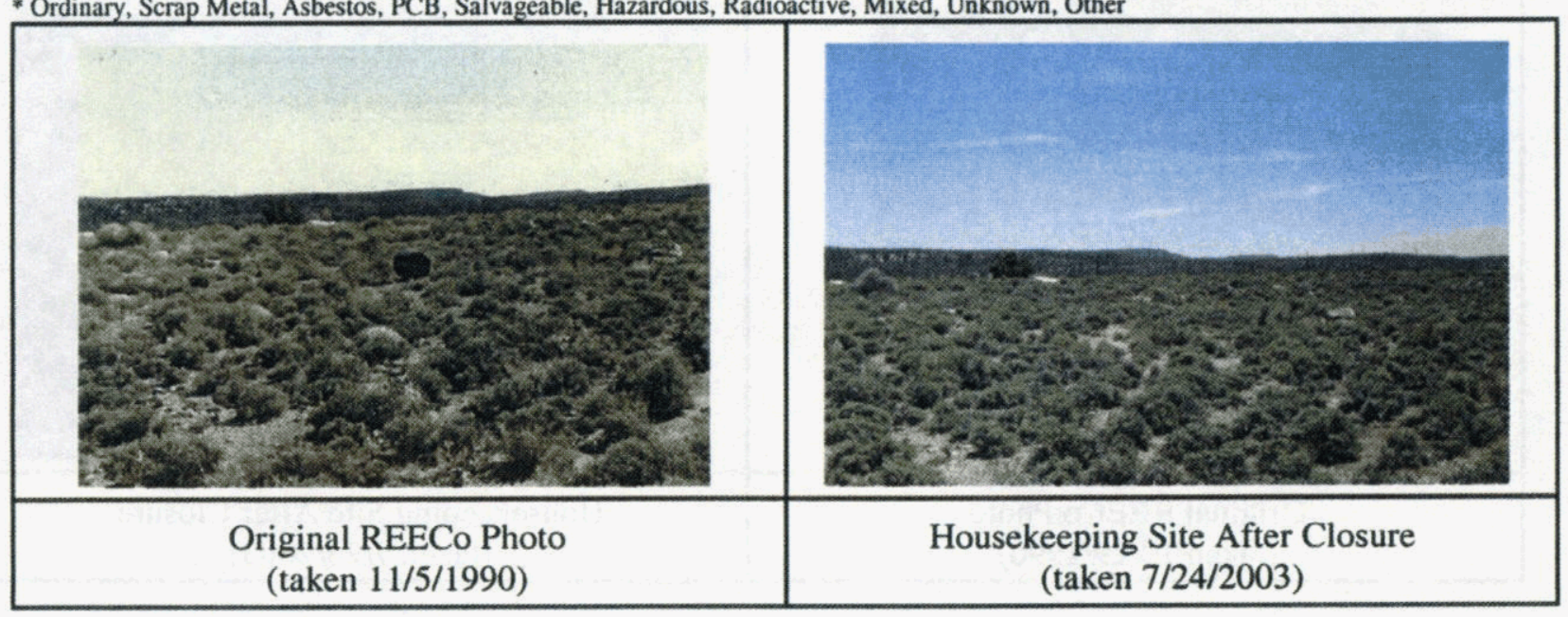

Current Site Description/Observations: No waste remained at this site during the Preliminary

Assessment or the Bechtel Nevada closure activities. The site is currently clear of all waste material.

$\mathbf{X}$ No Further Action Required at Housekeeping Site
Reed J. Poderis, CEM 


\section{Sectored Housekeeping Site Closure Verification Form}

Closure Verification Date: 7/24/2003

CAU Number: 523

CAS Number: $20-22-20$

CAS Description: Drum

Sector Designation: NTS Sector G

Housekeeping Site General Location: Nevada Test Site, U-20az

Elevation: 2,023 meters (m)

Northing: 4,120,542 m (UTM Zone 11)

Latitude: 37.23176

Easting: $552,421 \mathrm{~m}$ (UTM Zone 11)

Longitude: -116.40906

Coordinate/Elevation Data Obtained from: North American Datum, 1927.

Site Access Route: Take Mercury Highway north to Pahute Mesa Road. Turn left (west) on Pahute Mesa Road and proceed to Stockade Wash Road. Turn left (northwest) on Stockade Wash Road and proceed approximately 25 miles to Area 20. Proceed to a graded road at RadSafe Marker (RSM) 20 A 146, just before the Area 20 Camp. Turn left (south) on the graded road and continue approximately 3.7 miles to the U-20az Crater fence.

\begin{tabular}{|l|l|}
\hline \multicolumn{1}{|c|}{ Waste Item(s) Originally at Site } & \multicolumn{1}{c|}{ Apparent Waste Type* } \\
\hline One drum & None, drum was previously removed. \\
\hline
\end{tabular}

* Ordinary, Scrap Metal, Asbestos, PCB, Salvageable, Hazardous, Radioactive, Mixed, Unknown, Other

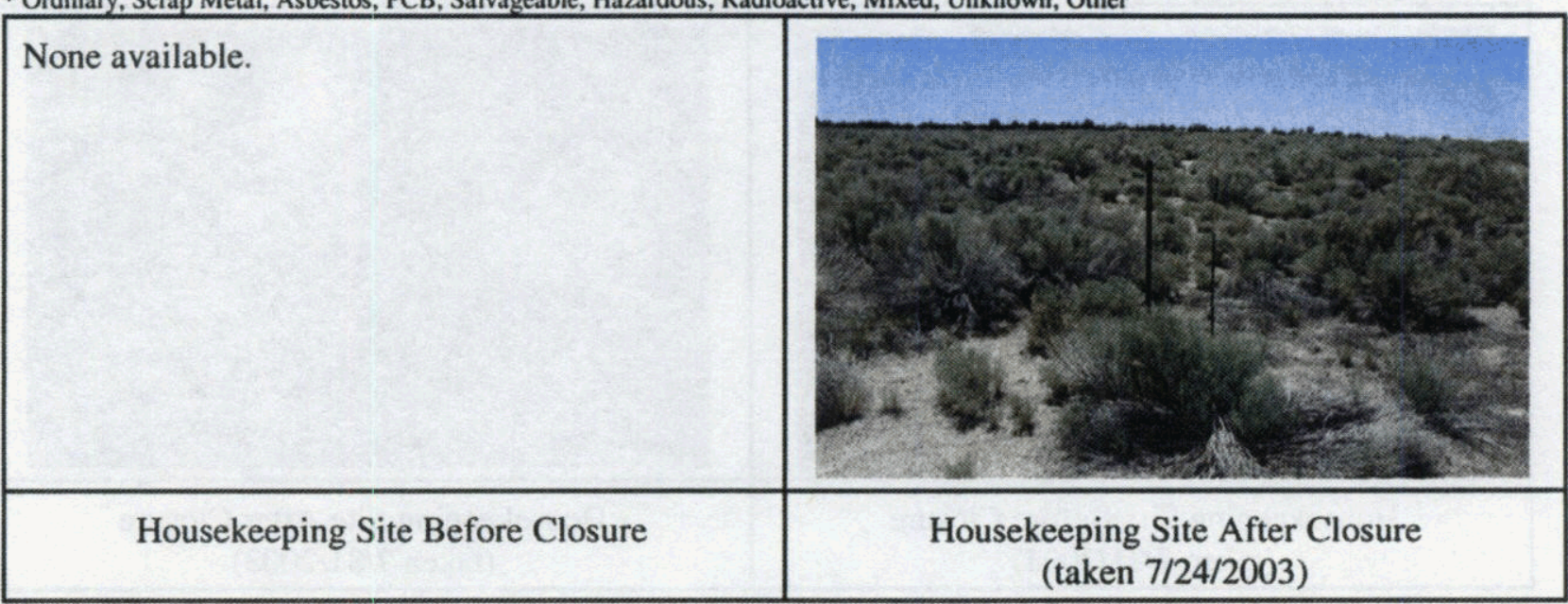

Current Site Description/Observations: No drum remained at this site during the Preliminary Assessment or the Bechtel Nevada closure activities. The site is currently clear of all waste material.

X No Further Action Required at Housekeeping Site

\begin{tabular}{cccc} 
Reed J. Poderis. CEM & $11-6-03$ \\
\hline Corrective Action Coordinator/Designee & Signature
\end{tabular}




\section{Sectored Housekeeping Site Closure Verification Form}

Closure Verification Date: 7/31/2003

CAU Number: 523

CAS Number: 20-22-27

CAS Description: Bucket

Sector Designation: NTS Sector G

Housekeeping Site General Location: Nevada Test Site, Area 20 Water Sump

Elevation: 1,996 meters $(\mathrm{m})$

Northing: 4,125,935 m (UTM Zone 11) Easting: 552,635 m (UTM Zone 11)

Latitude: $37.28036 \quad$ Longitude: -116.40627

Coordinate/Elevation Data Obtained from: North American Datum, 1927.

Site Access Route: Take Mercury Highway north to Tippipah Highway. Turn left (west) on Tippipah Highway and proceed to Pahute Mesa Road. Turn left (west) on Pahute Mesa Road and proceed to RadSafe Marker (RSM) P-106. Turn north on the dirt road just past the guard rail and continue to a large wooden shack (Water Well \#9). The site is $120 \mathrm{ft}$ east of the wooden shack.

\begin{tabular}{|l|l|}
\hline \multicolumn{1}{|c|}{ Waste Item(s) Originally at Site } & \multicolumn{1}{c|}{ Apparent Waste Type* } \\
\hline Two buckets, one empty drum. & Ordinary. \\
\hline
\end{tabular}

\begin{tabular}{|c|c|c|}
\hline * Ordinary, Scrap Metal, Asbestos, PCB, Salvageable, Hazardous, Radioactive, Mixed, Unknown, Other \\
\hline \\
\hline $\begin{array}{c}\text { Housekeeping Site Before Closure } \\
\text { (taken } 7 / 31 / 2003)\end{array}$
\end{tabular}

Current Site Description/Observations: The buckets and drum were disposed of in the Area 9 U-10c Landfill as ordinary waste. The site is currently clear of all waste material.

X No Further Action Required at Housekeeping Site

Reed J. Poderis, CEM

Corrective Action Coordinator/Designee

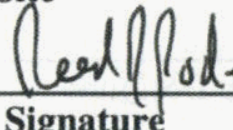

Signature
$11-6-0)$

Date 


\section{Sectored Housekeeping Site Closure Verification Form}

Closure Verification Date: 7/31/2003

CAU Number: 523

CAS Number: 20-22-28

CAS Description: Drums

Sector Designation: NTS Sector G

Housekeeping Site General Location: Nevada Test Site, U-20ax

Elevation: 1,981 meters $(\mathrm{m})$

Northing: 4,120,689 m (UTM Zone 11) Easting: 549,312 m (UTM Zone 11)

Latitude: 37.23325

Longitude: -116.4441

Coordinate/Elevation Data Obtained from: North American Datum, 1927.

Site Access Route: Take Mercury Highway north to Tippipah Highway. Turn left (northwest) on Tippipah Highway and proceed to Pahute Mesa Road. Turn left (west) on Pahute Mesa Road and travel 9.5 miles to Airport Road. Turn left (west) on Airport Road, which becomes Buckboard Mesa Road, and continue 17.0 miles to Pahute Mesa Road. Turn right (northeast) on Pahute Mesa Road and proceed to U-20ax. Turn right (southeast) and continue to the U-20ax Yard. The drums are located and marked on the left (south) side of the road.

\begin{tabular}{|l|l|}
\hline \multicolumn{1}{|c|}{ Waste Item(s) Originally at Site } & \multicolumn{1}{c|}{ Apparent Waste Type* } \\
\hline Three drums. & Ordinary. \\
\hline
\end{tabular}

" Ordinary, Scrap Metal, Asbestos, PCB, Salvageable, Hazardous, Radioactive, Mixed, Unknown, Other

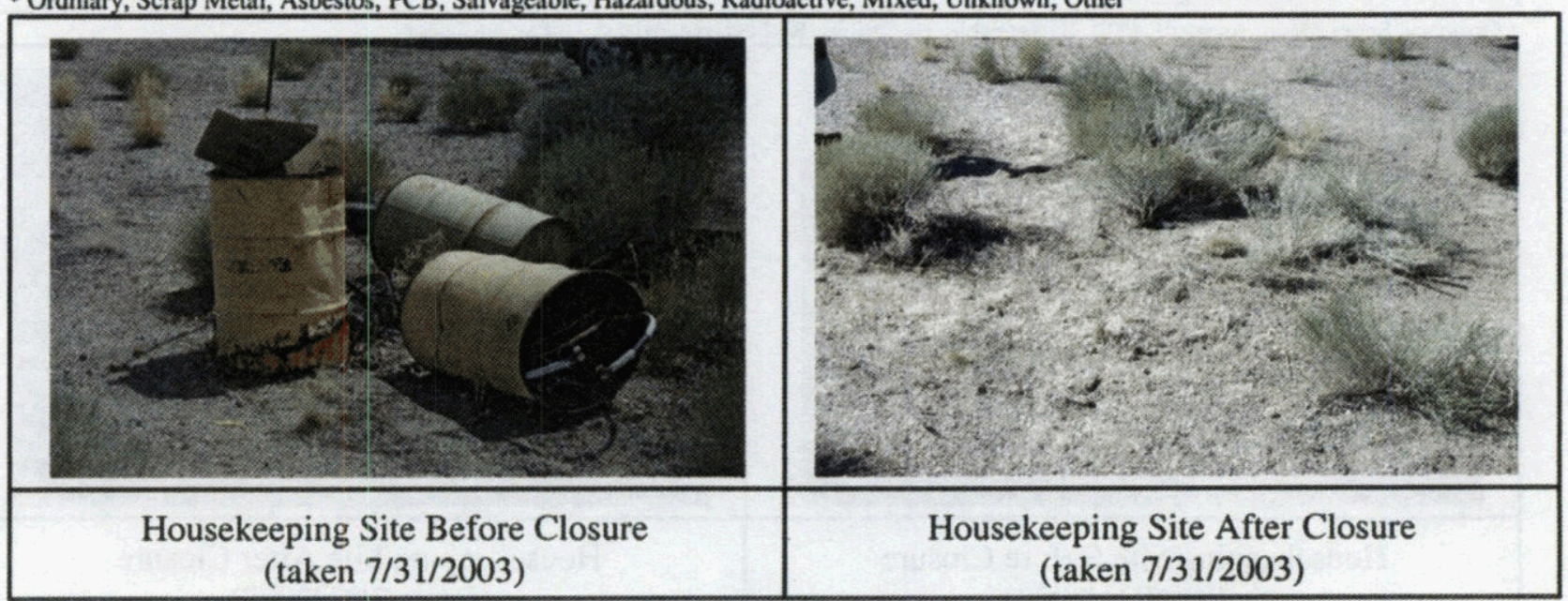

Current Site Description/Observations: The drums were disposed of in the Area 9 U-10c Landfill as ordinary waste. The site is currently clear of all waste material.

X No Further Action Required at Housekeeping Site

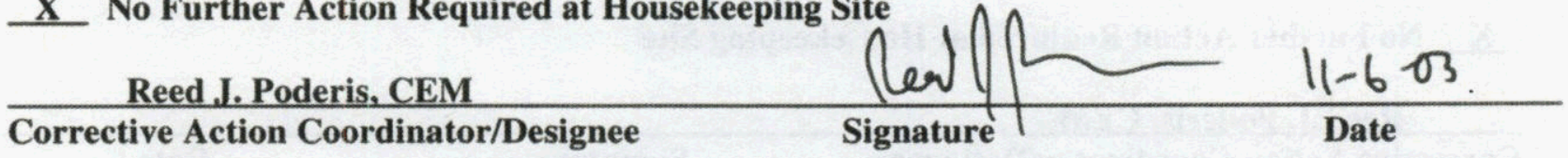




\section{Sectored Housekeeping Site Closure Verification Form}

Closure Verification Date: 7/31/2003

CAU Number: 523

CAS Number: $20-22-29$

CAS Description: Bucket

Sector Designation: NTS Sector G

Housekeeping Site General Location: Nevada Test Site, U-20ar

Elevation: 1,938 meters (m)

Northing: 4,128,993 m (UTM Zone 11) Easting: 546,489 m (UTM Zone 11)

Latitude: 37.30825

Longitude: -116.4754

Coordinate/Elevation Data Obtained from: North American Datum, 1927.

Site Access Route: Take Mercury Highway north to Pahute Mesa Road. Turn left (west) on Pahute Mesa Road and proceed to Airport Road. Turn left (west) on Airport Road, which turns into Buckboard Mesa Road, and proceed to Pahute Mesa Road. Turn right (northeast) on Pahute Mesa Road and proceed to 20-01 Road, at the south end of the Area 20 Camp. Turn left (northeast) on 20-01 Road and proceed 4.4 miles past M Road and J Road, toward the U-20ar crater. The bucket and site marker are on the left side of the road, near a high voltage power line.

\begin{tabular}{|l|l|}
\hline \multicolumn{1}{|c|}{ Waste Item(s) Originally at Site } & \multicolumn{1}{c|}{ Apparent Waste Type* } \\
\hline One intact bucket of grease. & Other, Hydrocarbon Waste. \\
\hline
\end{tabular}

* Ordinary, Scrap Metal, Asbestos, PCB, Salvageable, Hazardous, Radioactive, Mixed, Unknown, Other

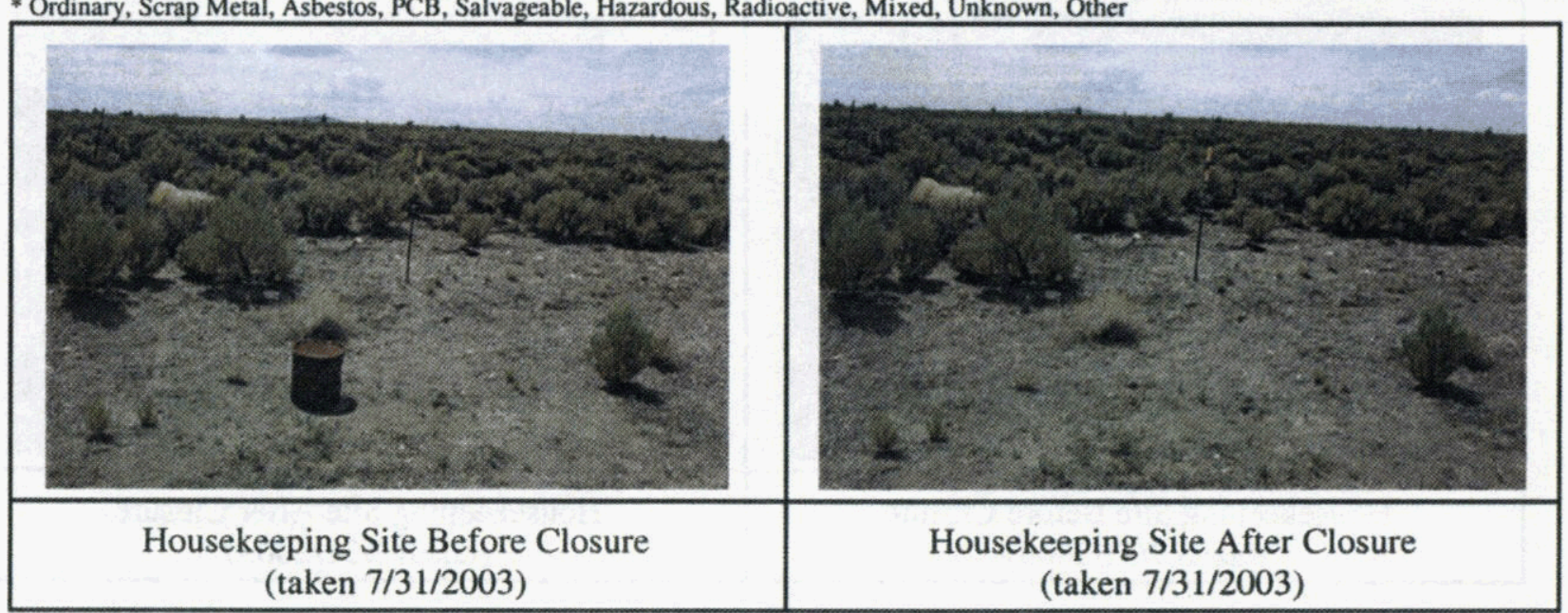

Current Site Description/Observations: The bucket was disposed of in the Area 6 Hydrocarbon Landfill as hydrocarbon waste. The site is currently clear of all waste material.

X No Further Action Required at Housekeeping Site

Reed J. Poderis, CEM

Corrective Action Coordinator/Designee

Signature

Date 


\section{Sectored Housekeeping Site Closure Verification Form}

Closure Verification Date: 7/24/2003

CAU Number: 523

CAS Number: 20-26-01

CAS Description: Lead Pellets

Sector Designation: NTS Sector G

Housekeeping Site General Location: Nevada Test Site, U-20ar

Elevation: 1,926 meters (m)

Northing: 4,129,762 m (UTM Zone 11)

Latitude: 37.31517

Easting: $546,739 \mathrm{~m}$ (UTM Zone 11)

Longitude: -116.47253

Coordinate/Elevation Data Obtained from: North American Datum, 1927.

Site Access Route: Take Mercury Highway north to Tippipah Highway. Turn left (west) on Tippipah Highway and proceed to Pahute Mesa Road. Turn left (west) on Pahute Mesa Road and proceed to Airport Road. Turn left (west) on Airport road, which turns into Buckboard Mesa Road, and proceed to Pahute Mesa Road. Turn left (west) on Pahute Mesa Road and proceed to M Road. Turn right (east) on M Road and proceed to 20-01 Road. Turn left (northwest) on 20-01 Road and continue to the area south of U-20aj. Take the branch of the road that initially heads west and follow it as it curves back north to the U-20ar crater.

\begin{tabular}{|l|l|}
\hline \multicolumn{1}{|c|}{ Waste Item(s) Originally at Site } & \multicolumn{1}{c|}{ Apparent Waste Type* } \\
\hline Several ripped bags of lead shot. & Salvageable, Hazardous. \\
\hline
\end{tabular}

* Ordinary, Scrap Metal, Asbestos, PCB, Salvageable, Hazardous, Radioactive, Mixed, Unknown, Other

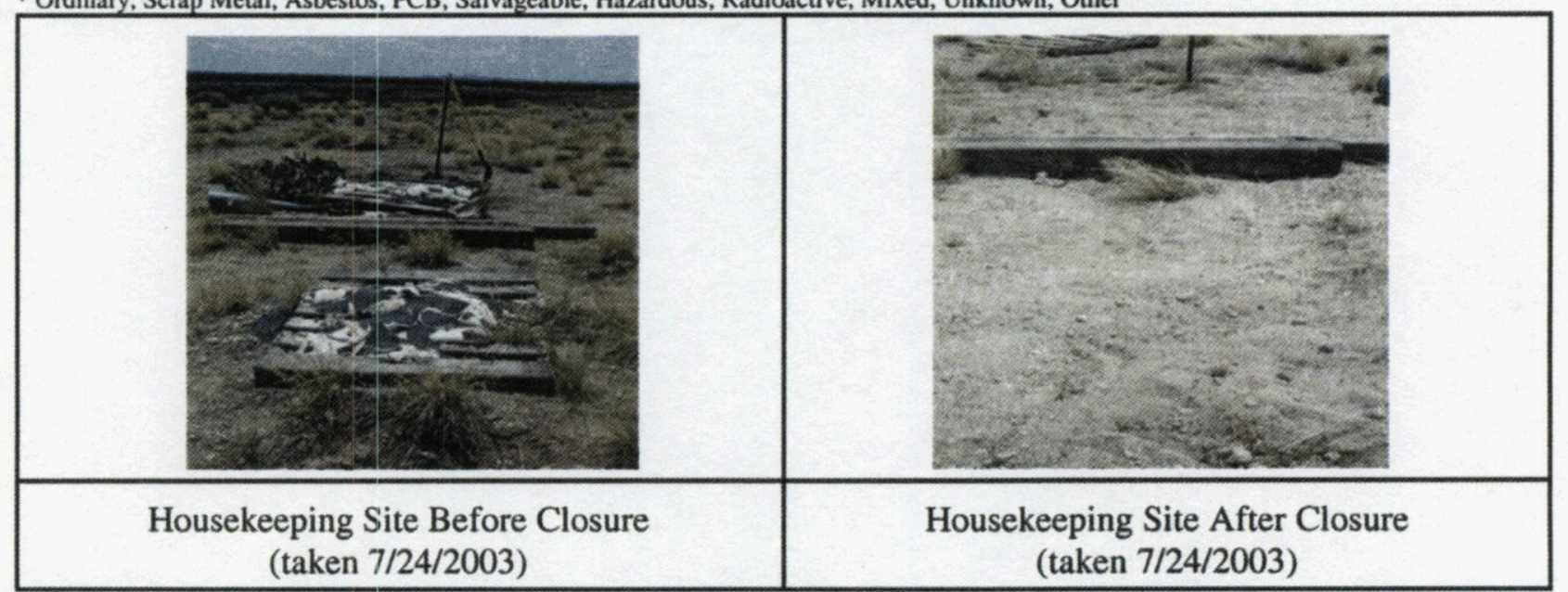

Current Site Description/Observations: One drum of segregated lead shot pellets were sent to the Excess Yard for salvage, and one drum of lead pellets and soil was disposed of off-site as hazardous waste. The site is currently clear of all waste material.

X No Further Action Required at Housekeeping Site

Reed J. Poderis, CEM

Corrective Action Coordinator/Designee

Signature

Date

B-15 
THIS PAGE INTENTIONALLY LEFT BLANK

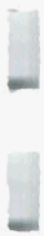

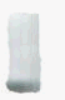

1

17

$-$

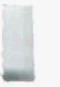

$-$

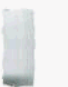




\section{APPENDIX C}

\section{NATIONAL ENVIRONMENTAL POLICY ACT EVALUATION CHECKLIST}


Closure Report - CAU 523

Section: Appendix C

Revision: 0

Date: November 2003

THIS PAGE INTENTIONALLY LEFT BLANK 


\section{U.S. DEPARTMENT OF ENERGY \\ NATIONAL NUCLEAR SECURITY ADMINISTRATION NEVADA OPERATIONS OFFICE NEPA ENVIRONMENTAL EVALUATION CHECKLIST}

\begin{tabular}{|l|l|}
\hline \multicolumn{1}{|c|}{ FOLLOW ATTACHED PROCEDURES FOR COMPLETING CHECKLIST } \\
$\begin{array}{l}\text { A. Project/Activity Title (Attach a brief description of proposed project) } \\
\text { CAUs 176, 346, 350, 351, 352 \& 523: Housekeeping Sites Closure/Cleanup Activities }\end{array}$ & $\begin{array}{l}\text { Date } \\
11 / 27 / 02 \text { (Amended 3/13/03) }\end{array}$ \\
\cline { 2 - 3 } & Proposed By (If other than NNSANV) \\
\hline $\begin{array}{l}\text { Project Location } \\
\text { NTS, Various Areas }\end{array}$ & $\begin{array}{l}\text { NNSANV Project/Program Manager } \\
\text { Kevin Cabble }\end{array}$ \\
\hline $\begin{array}{l}\text { NNSANV Line Management Organization } \\
\text { Environmental Restoration Division }\end{array}$ & DOE/HQ Program Office (If applicable) \\
\hline $\begin{array}{l}\text { Anticipated Start Date } \\
\text { January 8, 2003 }\end{array}$ & \\
\hline
\end{tabular}

ENVIRONMENTAL CONSIDERATIONS: If any phase of the projectlactivity involves any of the following considerations, check yes and explain in project description. See NV-16A for consideration guidelines and examples.

\begin{tabular}{|c|c|c|c|c|c|c|c|}
\hline Consideration & Yes & No & Unk & Consideration & Yes & No & Unk \\
\hline 1. Noise & $\bar{x}$ & & & 10. Liquid Effluents & & $\mathbf{x}$ & \\
\hline 2. Air Emissions & & $\mathbf{x}$ & & 11. Underground Storage/Septic Tanks & & $\mathbf{X}$ & \\
\hline 3. Explosives & $\bar{x}$ & & & 12. Utility Systems (PCBs) & $\bar{x}$ & & \\
\hline 4. Petroleum/Fuel Storage/Use & $\bar{x}$ & & & 13. Environmental Restoration Site & $\bar{x}$ & & \\
\hline 5. Pesticide/Herbicide Use & & $\underline{x}$ & & 14. Change in Existing Drainage Pattern & & $\bar{x}$ & \\
\hline 6. Hazardous/Toxic Substances & $\bar{x}$ & & & 15. Surface Disturbance/Excavation & $\bar{x}$ & & \\
\hline 7. Solid Waste & $\underline{x}$ & & & 16. Cultural/Historic Resources & & & $\bar{x}$ \\
\hline 8. Mixed Waste & & $\underline{\bar{x}}$ & & 17. Biological/Tortoise Resources & & $\underline{x}$ & \\
\hline 9. Radioactive Materials & $\bar{x}$ & & & & & & \\
\hline
\end{tabular}

9. Radioactive Materials

DO NOT TYPE OR WRITE BELOW THIS LINE, FORESHD USE ONLY.

B. Is the project/activity included in the final NTS EIS and the ROD or other NEPA document? Yes $\mathrm{X}$ (complete Sections C, D, and E) No (complete Sections D, E, and F)

C. This project/activity is included in the NTS EIS/ROD (or other NEPA document) under the following section and page number:

NTS EIS Volume 1, Appendix A, A.3.1.3 - Environmental Restoration Program; Industrial Sites Project

D. Does the proposed project/activity require any local, state, or federal permits or notifications?

Yes $\underline{X} \quad$ No

E. Does the proposed project/activity relate to the FFCA or FFACO agreements?

Yes $\mathrm{X} \quad$ No

F. If, based on the project description and the preliminary environmental considerations noted above, the proposed action fits within a class of action listed in Subpart D of 10 CFR 1021, write in the space below, the paragraph number and short title from the appropriate table of contents of Subpart D, Appendix B, C, or D, for a CX, EA, or EIS. If the proposed action does not fit within any class of action, write "Not Listed" below.

\section{G. NEPA COMPLIANCE OFFICER DETERMINATION OR RECOMMENDATION:}

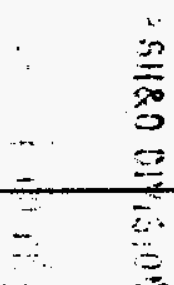

I have determined that the proposed action as described in Item $A$ above, has been adequately addressed for the purpose of NEPA in the NTS EIS. No further analysis or documentation is required pursuant to NEPA.
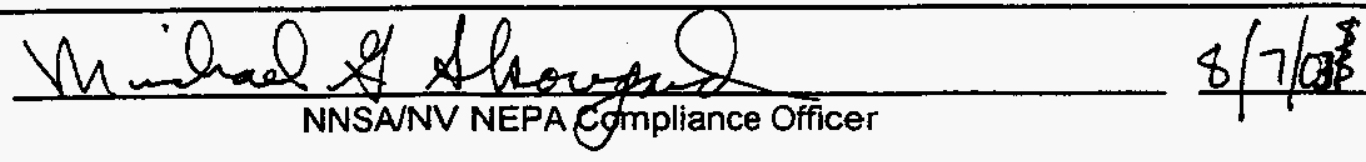


\section{CAUS 176, 346, 350, 351, 352 \& 523: HOUSEKEEPING SITES CLOSURE/CLEANUP ACTIVITIES}

\section{Project Description}

All CAUs are housekeeping sites, located at the Nevada Test Site (NTS). CAU 176 is located in Areas 5 and 6 and consists of four Corrective Action Sites (CASs). CAU 346 is located in Areas 8 and 10 and consists of 13 CASs. CAU 350 is located in Area 15 and consists of nine CASs. CAU 351 is located in Areas 18 and 30 and consists of six CASs. CAU 352 is located in Areas 19 and 20 and consists of 14 CASs. CAU 523 is located in Areas 4, 12, 16, 18, 19, and 20 and consists of 18 CASs.

The purpose of this project is to remove the various debris located at each CAS. A detailed list of the CASs and descriptions is provided in the FFACO (1996). Each CAU is listed below, with a brief description of the types of debris present in the CASs.

\section{CAU 176}

Includes two small vacuums, an automotive battery, oil can, 330-gallon tank filled with sanitary trash, and scrap wood and metal. The vacuums and possibly the wood and metal debris are radioactive.

\section{CAU 346}

The majority of debris at the CASs comprising CAU 346 appear to be drums filled with concrete that have electrical cables running out from the concrete and extending to various boreholes. Other debris include hoses, scrap metal, wood debris, cables, wire, etc. Portions of these wastes may be radioactive.

\section{CAU 350}

Wastes at these CASs include metal and wood debris, empty fuel cans, several tins that are labelled "Blasting Caps," a deteriorated dry cell battery, and a gas cylinder approximately $4 \mathrm{ft} \times 1 \mathrm{ft}$. None of the wastes are radiological, but the blasting cap tins and gas cylinder may be hazardous or toxic.

\section{CAU 351}

The majority of debris at these CASs includes dry cell batteries. Other waste includes wood, metal and glass debris, and two rusted, green jeeps. Some of the waste may be radioactive and or hazardous/toxic.

\section{CAU 352}

Debris includes a transformer, wood, rusted metal pipes, PVC pipes, chunks of concrete, broken batteries, empty cans and drums, and lead pellets. There is also a black stained area at one of the locations.

\section{CAU 523}

Debris includes wood, rusted metal pipes, broken batteries, aerosol cans, a grease bucket, lead pellets, and empty cans and drums.

\section{Environmental Considerations}

1. Noise levels: Elevated noise levels may result from the operation of a backhoe and/or loader equipment. Personnel not directly involved with operation of this equipment will be kept back at least 15 feet while equipment is in use. The equipment operator will follow the instructions as directed in the Site Specific Health and Safety Plan.

3. Explosives: Several tins marked "Blasting Caps" will be exploded in place. The certified blaster will direct all operations while during this phase of the cleanup.

4. Petroleum/Fuel Storage Use: Heavy equipment on site will use petroleum fuel. No fuel will be stored on site outside of the equipment. Absorbent pads will be used if equipment appears to be leaking petroleum.

6. Hazardous/Toxic Substances: Several sites contain lead, PCB or TPH-impacted and/or sanitary waste and soil. No other chemical hazards are known to exist. Personnel will be required to follow the safety procedures outlined in the Site Specific Health and Safety Plan and Job Hazard Analysis. Wastes will be disposed of either on the NTS or offsite, depending on analysis results. 
9. Radioactive Materials: Several sites are in a URMA, CA, or RMA. Radiologically-impacted soil and manufactured items in these areas may require disposal at either the Area 3 or Area 5 Radioactive Waste Management Site.

12. Utility Systems (PCBs): Two sites contain transformers which are potentially contaminated with PCBs. These will be disposed of as PCB waste if found to contain PCBs. Personnel will be required to follow the safety procedures outlined in the Site Specific Health and Safety Plan and Job Hazard Analysis.

13. Environmental Restoration Site: These sites are included in the FFACO as Corrective Action Unit $176,346,350,351$, 352, and 523. (See Project Description).

- 15. Surface Disturbance/Excavation: No excavations are planned. Equipment will travel off-road on previously disturbed areas. Equipment will also be used to help remove surface debris.

16. Cultural/Historic Resources: Several sites contain equipment and debris of mining activities which occurred before the Nevada Test Site was removed from the public domain. These artifacts will be left undisturbed. 


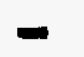

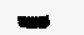

w.

THIS PAGE INTENTIONALLY LEFT BLANK

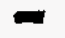

$-$

$-$

$-$

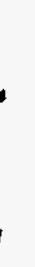


Closure Report - CAU 523

Section: Appendix D

Revision: 0

Date: November 2003

\section{APPENDIX D}

\section{DOCUMENTATION FOR CAS 12-30-16: MANHOLE FOR GAS/AIR LINE}


Closure Report - CAU 523

Section: Appendix D

Revision: 0

Date: November 2003
$-$

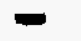

$-$

-

$-$

$=$

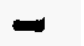

$-$

$-$

$\rightarrow$

THIS PAGE INTENTIONALLY LEFT BLANK
$-$

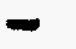

$-$

$-$

$-$

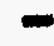

$-$

$-$ 


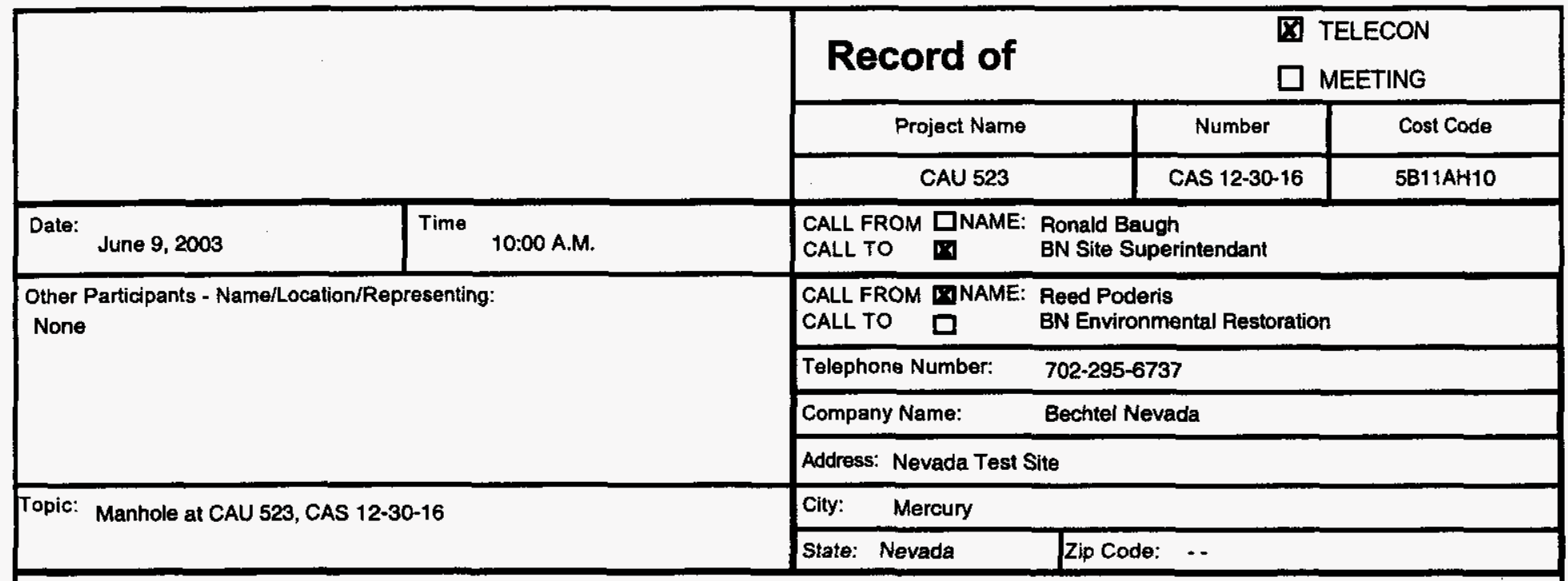

Summary (Decisions \& Specific Actions Required by Named Persons):

The manhole identified in the FFACO as CAU 523, CAS 12-30-16 is an air/vacuum release valve which is located on the deactivated 12-2 Water Transmission Line that runs from Area 12 to Area 2. The manhole is a utility and therefore should not be considered waste. It is also related to a water line and therefore no environmental hazards are associated with the line itself or the release valve.

Mr. Baugh stated that the manhole and/or valve assemble may not be removed as it is utility property. The valve itself is identified on the attached As-Built diagrams and summarized below:

JS-002-083-C3.1 Site Plan (depicts the overall plan view of the water line.

JS-002-083-C6 Plan \& Profile - 30+00 to 60+00 (depicts the pertinent section as well as the valve location itself)

JS-002-083-C13.1 Details (depicts the layout of a typical air/vacuum release valve and associated manhole.

Copies of the relevant diagrams were obtained and are attached.

\section{Required Action:}

As this CAS is not considered by the Utility Department to be waste, it should be removed from the FFACO and be exempt from the cleanup activities for CAU 523.

Signature and Date to affirm Concurrence of Participants (as appropriate):

\begin{tabular}{l|l}
\hline DOEINSO & Shaw Environmental, Inc.
\end{tabular}

\section{Shaw Environmental, Inc.}

(10

Distribution:

Original to Project File

Copy to Project Manager

Copy to Preparer

O Other Distribution (By Preparer)

Environmental Restoration 


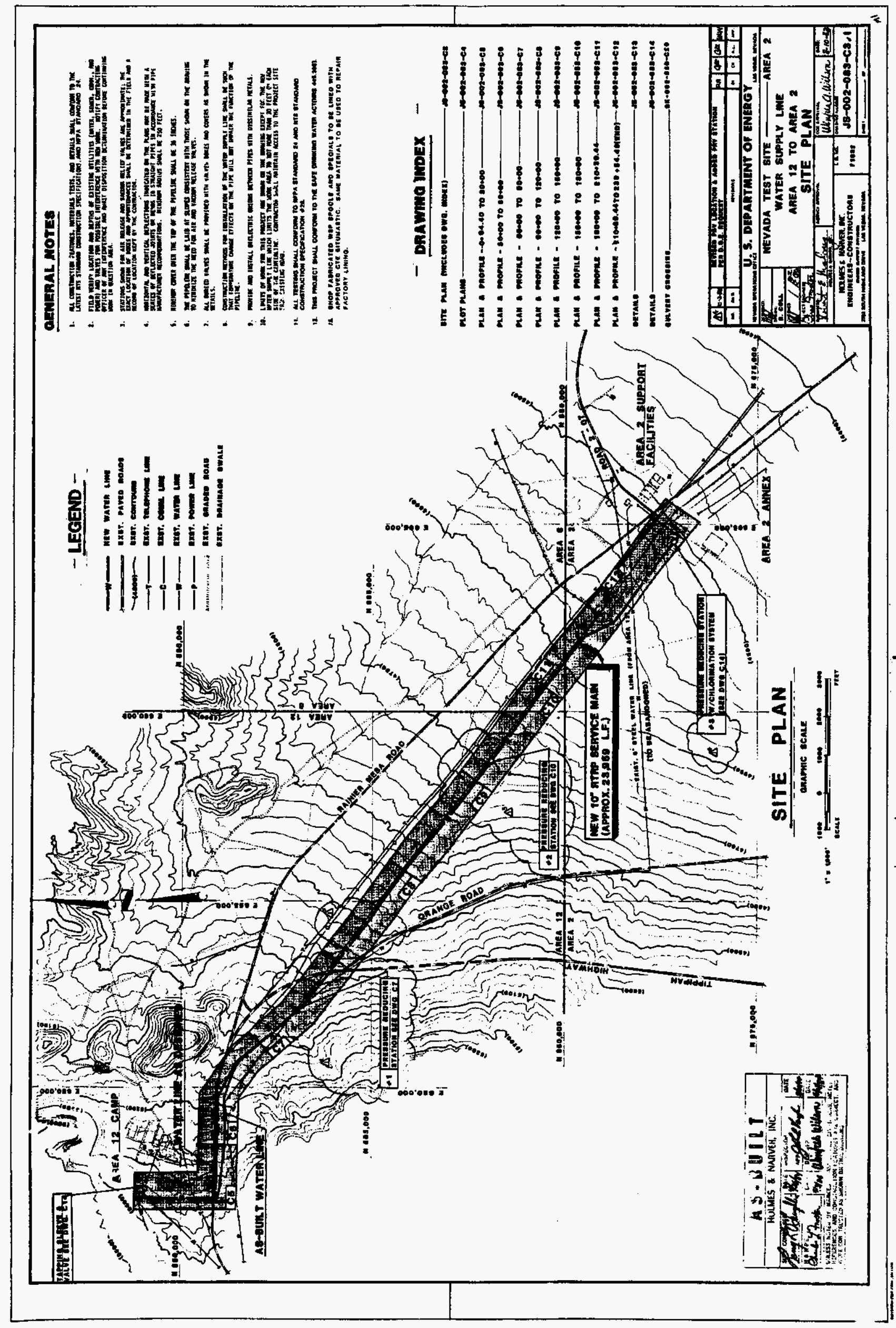




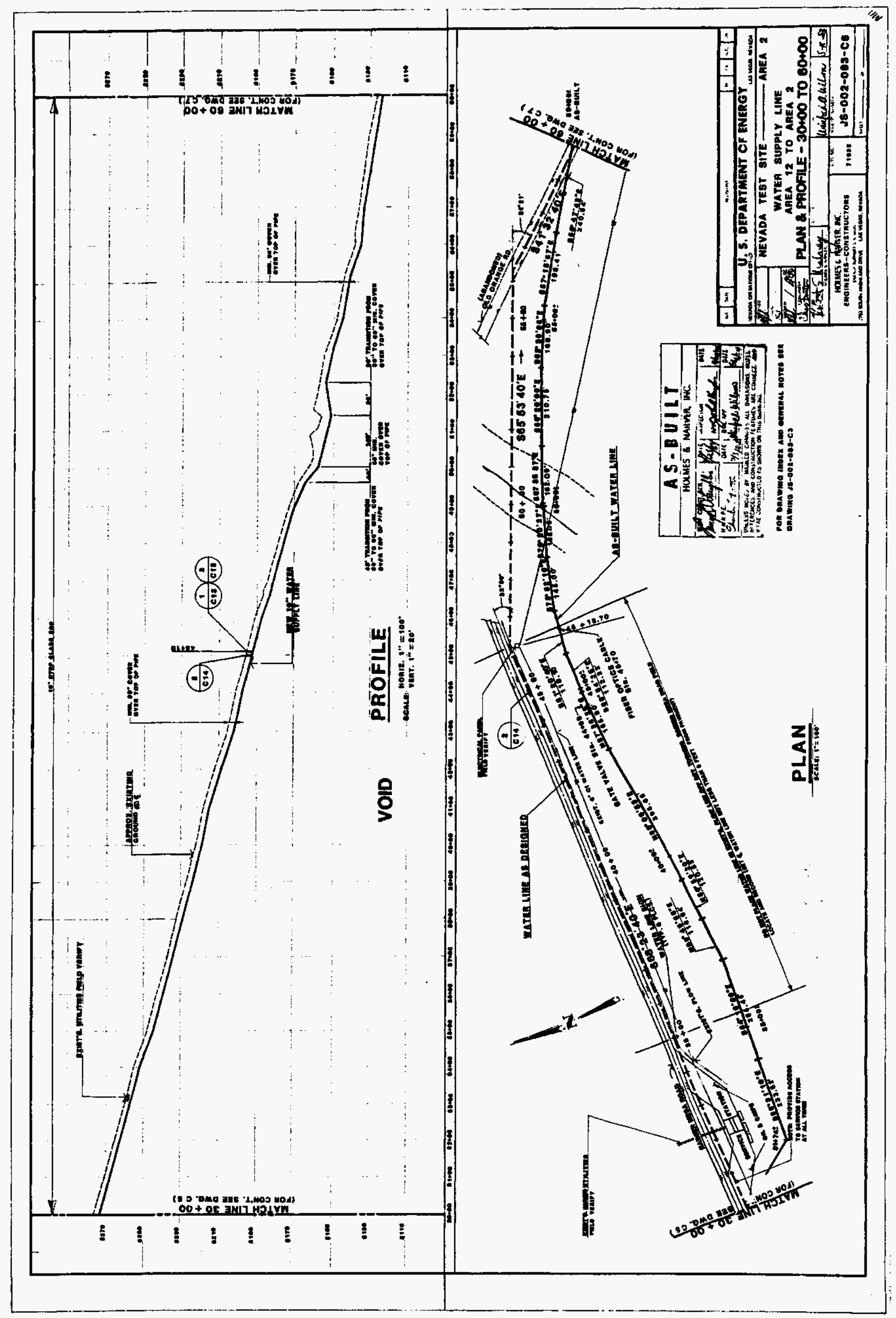




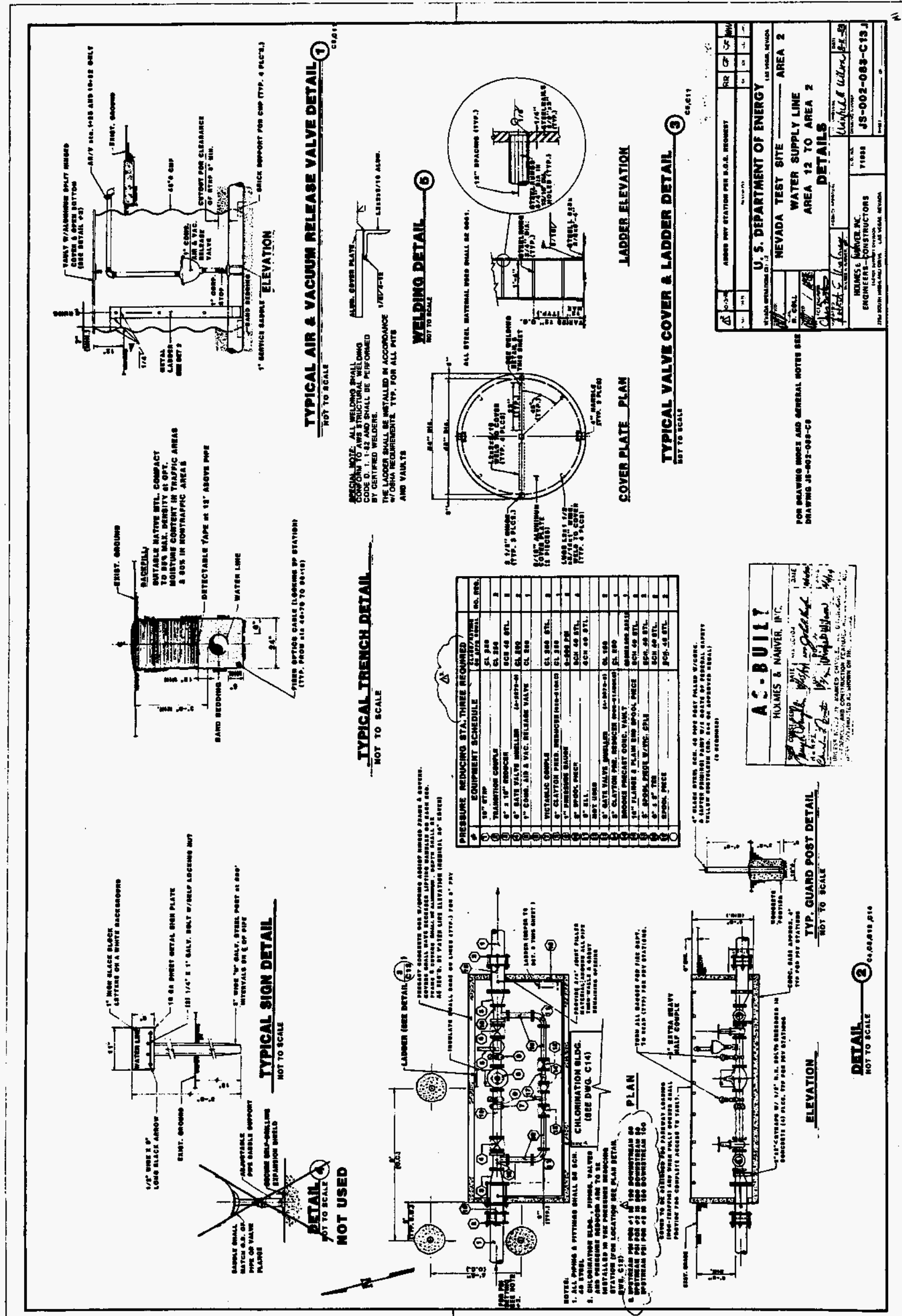


Closure Report - CAU 523

Section: Distribution List

Revision: 0

Date: November 2003

\section{DISTRIBUTION LIST}


Closure Report - CAU 523

Section: Distribution List

Revision: 0

Date: November 2003

THIS PAGE INTENTIONALLY LEFT BLANK 


\section{DISTRIBUTION LIST}

*Provide copy of initial distribution of all revisions; others receive NDEP-approved revision only.

\section{Nevada Division of Environmental Protection}

Paul Liebendorfer

1 (Controlled)*

Bureau of Federal Facilities

Division of Environmental Protection

333 W. Nye Lane, Room 138

Carson City, NV 89706-0866

Donald Elle

1 (Controlled)*

Bureau of Federal Facilities

Division of Environmental Protection

1771 E. Flamingo Rd., Suite 121-A

Las Vegas, NV 89119-0837

\section{U.S. Department of Energy}

Janet Appenzeller-Wing

1 (Uncontrolled)*

Environmental Restoration Division

U.S. Department of Energy

National Nuclear Security Administration

Nevada Site Office

P.O. Box $98518, \mathrm{M} / \mathrm{S} 505$

Las Vegas, NV 89193-8518

Kevin Cabble

1 (Uncontrolled)*

Environmental Restoration Division

U.S. Department of Energy

National Nuclear Security Administration

Nevada Site Office

P.O. Box 98518, M/S 505

Las Vegas, NV 89193-8518

Shirley Doty

1 (Controlled)*

Environmental Restoration Division

U.S. Department of Energy

National Nuclear Security Administration

Nevada Site Office

P.O. Box $98518, \mathrm{M} / \mathrm{S} 505$

Las Vegas, NV 89193-8518 


\section{DISTRIBUTION LIST (continued)}

\section{U.S. Department of Energy (continued)}

U.S. Department of Energy

National Nuclear Security Administration

Nevada Site Office

Southern Nevada Public Reading Facility

$\mathrm{C} / \mathrm{O}$ Nuclear Testing Archive

P.O. Box 98521, M/S 400

Las Vegas, NV 89193-8521

U.S. Department of Energy

National Nuclear Security Administration

Nevada Site Office

Technical Library

P.O. Box 98518

Las Vegas, NV 89193-8518

U.S. Department of Energy

Office of Scientific and Technical Information

P.O. Box 62

Oak Ridge, TN 37831-0062

\section{Bechtel Nevada}

Correspondence Control

1 (Uncontrolled)*

Bechtel Nevada

P.O. Box 98521, M/S CF008

Las Vegas, NV 89193-8521

Environmental Management Library

1 (Uncontrolled)*

Bechtel Nevada

P.O. Box 98521, M/S NLV080

Las Vegas, NV 89193-8521

Kevin Campbell

1 (Uncontrolled)*

Bechtel Nevada

P.O. Box 98521, M/S NTS306

Las Vegas, NV 89193-8521

Reed Poderis

Bechtel Nevada

P.O. Box 98521, M/S NTS306

Las Vegas, NV 89193-8521
1 (Controlled) \&

1 (Uncontrolled)

1 (Uncontrolled) 


\section{DISTRIBUTION LIST (continued)}

\section{Bechtel Nevada (continued)}

Steve Nacht

1 (Uncontrolled)*

Bechtel Nevada

P.O. Box 98521, M/S NTS306

Las Vegas, NV 89193-8521

Allison Urbon

1 (Uncontrolled)*

Bechtel Nevada

P.O. Box 98521, M/S NTS306

Las Vegas, NV 89193-8521

\section{Nve County}

David Swanson

1 (Uncontrolled)* \&

Nye County

Department of Natural Resources \& Federal Facilities

1 (Uncontrolled electronic)

1210 East Basin Road, Suite 6

Pahrump, NV 89060

\section{State of Nevada}

Manager, Northern Nevada

1 (Uncontrolled)

FFACO Public Reading Facility

Nevada State Library and Archives Federal Publications

100 North Stewart Street

Carson City, NV 89701-4285

\section{Stoller-Navarro}

FFACO Coordinator

1 (Controlled)

Stoller-Navarro

7710 W. Cheyenne Ave.

Building 3

Las Vegas, NV 89129

Brian Hoenes

1 (Uncontrolled)*

Stoller-Navarro

7710 W. Cheyenne Ave.

Building 3

Las Vegas, NV 89129 
Revision: 0

Date: November 2003 